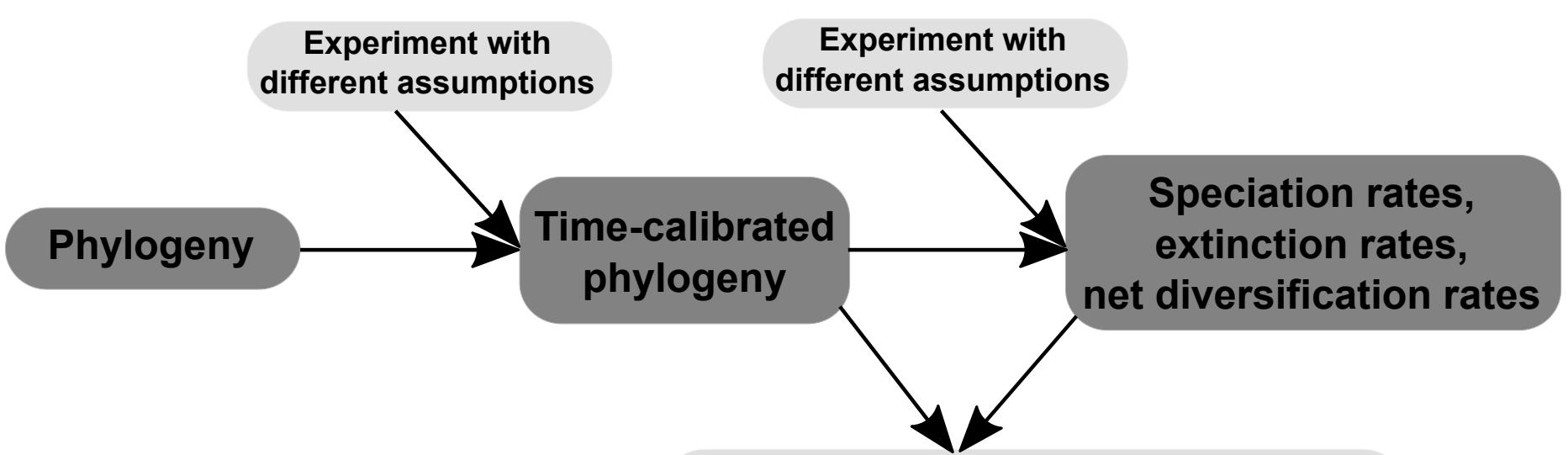

Key findings:

- Extremely uncertain inferences

- Major increase in net diversification rates for a neotropical clade of Ipomoea

- The origin of the sweet potato was not a result of human domestication 


\section{The temporal dynamics of evolutionary diversification in Ipomoea}

Tom Carruthers ${ }^{1}$, Pablo Muñoz-Rodríguez ${ }^{1}$, John R. I. Wood ${ }^{1}$, and Robert W.

Scotland ${ }^{*}$

\section{Affiliations:}

${ }^{1}$ Department of Plant Sciences, University of Oxford, South Parks Road, Oxford, OX1 3RB, United Kingdom

* Correspondence to be sent to: Department of Plant Sciences, University of Oxford, South

Parks Road, Oxford, OX1 3RB, United Kingdom; E-mail: robert.scotland@plants.ox.ac.uk 


\section{Abstract}

Molecular phylogenies are used as a basis for making inferences about macroevolutionary history. However, a robust phylogeny does not contain the information that is necessary to make many of these inferences. Complex methodologies that incorporate important assumptions about the nature of evolutionary history are therefore required. Here, we explore the implications of these assumptions for making inferences about the macroevolutionary history of Ipomoea - a large pantropical genus of flowering plants that contains the sweet potato (Ipomoea batatas), a crop of global economic importance. We focus on assumptions that underlie inferences of divergence times, and diversification parameters (speciation rates, extinction rates, and net diversification rates). These are among the most fundamental variables in macroevolutionary research. We use a series of novel approaches to explore the implications of these assumptions for inferring the age of Ipomoea, the ages of major clades within Ipomoea, whether there are significant differences in diversification parameters among clades within Ipomoea, and whether the storage root of I. batatas evolved in pre-human times. We show that inferring an age estimate for Ipomoea and major clades within Ipomoea is highly problematic. Inferred divergence times are sensitive to uncertain fossil calibrations and differing assumptions about among-branch-substitution-rate-variation. Despite this uncertainty, we are able to make robust inferences about patterns of variation in diversification parameters within Ipomoea, and that the storage root of I. batatas evolved in pre-human times. Taken together, this study presents novel and generalizable insights into the implications of methodological assumptions for making inferences about macroevolutionary history. Further, by presenting novel findings relating to the temporal dynamics of evolution in Ipomoea, as well as more specifically to I. batatas, this study makes a valuable contribution to our understanding of tropical plant evolution, and the evolutionary context in which economically important crops evolve.

Key words: phylogenetics, evolution, divergence times, diversification, Ipomoea, Sweet Potato 


\section{INTRODUCTION}

Molecular phylogenies are of central importance to macroevolutionary research. Our understanding of evolutionary radiations, and more generally, the factors that affect when, how, and why diversity accumulates in different clades, is underpinned by the interpretation of molecular phylogenies in an evolutionary context (Baldwin and Sanderson 1998; Hughes and Eastwood 2006; Givnish et al. 2009; Rabosky et al. 2008; Koenan et al. 2013; Donoghue and Sanderson 2015; Nevado et al. 2016; Rabosky et al. 2018). So too is our understanding of when and how the earth's major biomes were assembled (Pennington et al. 2009; Simon et al. 2009; Govindarajulu et al. 2011; Särkinen et al. 2012; Hughes et al. 2013; Pennington and Lavin 2015; Dexter et al. 2017) and the historical processes that have shaped distribution patterns such as long-distance-dispersal and geological vicariance (Lavin et al. 2004; Donoghue 2008; Crisp et al. 2011; Muñoz-Rodríguez et al. 2018). Because of their importance to macroevolutionary research, the rapid increase in availability of robust molecular phylogenies for many groups has enabled macroevolutionary questions to be investigated in greater detail and a greater variety of different contexts.

There are fundamental differences between a robust molecular phylogeny and the evolutionary process that it is used as a basis for making inferences about. These differences mean that even if a robust phylogeny is known for a group, parameters of interest in macroevolutionary research such as divergence times, net diversification rates, speciation rates, and extinction rates can be inferred with significant error (Sanderson and Doyle 2001; Britton 2005; Rabosky 2010; Moore et al. 2016; Wilf and Escapa 2015; Carruthers et al. 2019). It is crucial to account for this error, especially when using inferences as a basis for constructing narratives or explanations about when, how and why a group evolved. If error is not adequately accounted for, complex narratives may be constructed that are not supported by available evidence (Wilf and Escapa 2015).

Here, we use the recently developed phylogenetic framework for Ipomoea (MuñozRodríguez et al. 2019) to investigate the temporal dynamics of its evolutionary diversification. Ipomoea is a pantropical genus of over 800 species that exhibit a range of growth forms including climbers, trailers, erect shrubs and trees. They also occur in a range of habitats including cerrado, dry forest, and disturbed wasteland (Wood et al. 2015). Further, the genus contains the sweet potato (I. batatas), a crop of global economic importance (Muñoz-Rodríguez et al. 2018). Investigating the temporal dynamics of evolutionary diversification in Ipomoea can therefore provide critical insights into the nature of 
evolutionary diversification in large tropical groups, and when, how and why important crops evolve from their wild relatives.

In this study, we specifically focus on the inference of divergence times, net diversification rates, speciation rates, and extinction rates. Net diversification rates, speciation rates, and extinction rates are subsequently referred to collectively as diversification parameters. We focus on divergence times and diversification parameters because these two variables are the fundamental basis upon which other aspects of macroevolutionary research depend. When inferring these two variables, we explicitly consider different sources of uncertainty. With respect to divergence times, one of the primary sources of uncertainty are fossil calibrations (Sanderson and Doyle 2001; dos Reis and Yang 2013; Magallón et al 2013; Warnock et al. 2015; Morris et al. 2018). These are likely to be a particularly important source of uncertainty for Ipomoea, and many other angiosperm genera, because their fossil records are extremely fragmentary (Särkinen et al. 2013; Mitchel et al. 2016; Cardillo et al. 2017; Wilf et al. 2017; Folk et al 2019). It is therefore difficult to make robust interpretations of the temporal signal in the fossil record, and to use it as a basis to calibrate phylogenies. For divergence times, we also consider the effect of different assumptions about substitution rates and the magnitude of among-branchsubstitution-rate-variation (Zuckerkandl and Pauling 1962, 1965; Fitch 1976; Gillespie 1991; Sanderson 1997; Thorne et al. 1998; Britton 2005; Bromham 2006; Drummond et al. 2006; Zhu et al. 2015; Carruthers et al. 2019), as well as the effect of assuming constant diversification parameters when performing divergence time analyses in a Bayesian framework. For the inference of diversification parameters, we explore the effect of divergence time estimation error on inferred diversification parameters. We also discuss the effects of limited taxon sampling, and the effects of extinct branches that are un-sampled in a molecular phylogeny.

When exploring these sources of uncertainty in the context of Ipomoea, we investigate several questions that relate to the timing and tempo of evolutionary diversification in the entire genus. These include the age of Ipomoea, the ages of major clades within Ipomoea, diversification parameters within Ipomoea, and the extent to which diversification parameters vary among clades within Ipomoea. Given the recently developed phylogenetic framework for Ipomoea resolves the relationships between I. batatas and its closest wild relatives (Muñoz-Rodríguez et al. 2018), we also investigate questions relating to the origin of I. batatas. This includes when I. batatas and its storage root evolved, and 
specifically, whether it evolved in pre-human times, or whether it evolved more recently as a likely result of human domestication.

\section{MATERIALS AND METHODS}

\subsection{Estimating the age of Ipomoea}

To estimate the age of Iрomoea, we inferred a time-calibrated phylogeny for Convolvulaceae, which contains Ipomoea, and its sister family Solanaceae, which has a richer fossil record (Särkinen et al. 2013; Wilf et al 2017). This time-calibrated phylogeny was based on a molecular dataset of 4 chloroplast genes $(a t p B, m a t K, n d h F, r b c L)$, the chloroplast intergenic spacer trnL-trnF, and the nuclear ITS marker. Apart from sampled species of Ipomoea - for which we used sequences from Muñoz-Rodríguez et al. 2019, sampled sequences were downloaded from GenBank (Table S1). A total of 56 taxa were sampled to represent the phylogenetic breadth of the two families. We purposefully sampled a low proportion of taxa, and an equal number of taxa from each sister family. The rationale for this was to reduce the effect of violating the assumption of the constant rate birth-death branching process that the diversification parameters are constant - an underlying assumption of most Bayesian methods of divergence time estimation. This is because sampling an equal number of taxa from each sister clade will likely cause diversification parameters to be constant amongst sampled taxa at broad sales across the phylogeny, whilst sampling a small number of taxa will mean that any violation of the assumption of constant diversification parameters amongst sampled taxa will have a statistically weaker effect on divergence time estimates.

We aligned sequences in MAFFT v7.271 (Katoh 2002; Katoh and Standley 2013) using the L-INS-I setting and a gap opening penalty of 1.53. Ambiguously aligned regions were removed using default settings in Gblocks (Castresana 2000; Talavera and Castresana 2007), and Sequence Matrix v1.8 (Vaidya et al. 2011) was used to concatenate aligned sequences. The final concatenated matrix had 5,166 sites for the 56 taxa. We used MrBayes v3.2.6 (Huelesenbeck and Ronquist 2001; Ronquist et al. 2012) to infer the tree topology for subsequent divergence time analyses. A GTR + G + I model was used. This analysis was run for 2,000,000 generations and the final split frequency between runs was $<0.01$. The topology we recovered is consistent with previous phylogenetic studies of these two families (Stefanović et al. 2003; Särkinen et al. 2013; Simoes et al. 2015; Mitchell et al. 2016).

The validity of different temporal calibrations for Convolvulaceae and Solanaceae is unclear. We therefore experimented with different calibration strategies. However, in no case did we use any fossil calibrations within Ipomoea because there are no fossils that can 
reliably be assigned to the genus. This includes the recently published leaf fossils of Ipomoea meghalayensis (Srivastava et al. 2018). This is because the short thick petioles of these fossils are not typical of Ipomoea; their well-preserved gland dots, leaf venation, and cordate leaves are characteristic of several other genera; and from the fossils alone it cannot be determined that the plant has alternate leaves, as would be expected for Ipomoea (Hawthorne pers. comm.)

In Calibration Strategy 1, 2 and 3, we used the recently discovered fossil, Physalis infinemundi (Wilf et al. 2017). This fossil - which is at least 52 million-years-old - is far older than fossil calibrations previously used in Convolvulaceae and Solanaceae, and is also considerably older than previous age estimates for either of these two families (Särkinen et al. 2013; Magallón et al. 2015; Mitchell et al. 2016). Because of this, it places previous assumptions about the timing of evolution within these clades, and the relationship between fossil ages and clade ages, into considerable doubt.

In Calibration Strategy 1, both the Solanoideae stem node and Solanaceae stem node are calibrated with a uniform distribution $($ minimum $=52, \max =130)$. The minimum corresponds to the age of Physalis infinemundi, and the maximum corresponds to the time that Eudicot pollen suddenly appears in the fossil record (Doyle et al 1977). This calibration strategy assumes the Solanoideae stem node is older than 52 million years, and the Solanaceae stem node is younger than 130 million years. However, apart from this, it does not make explicit assumptions about how much older or younger than these minima or maxima these two nodes are likely to be.

In Calibration Strategy 2 and 3, we accounted for the fact that 130 million years is most probably an overestimate of the Solanaceae stem node age - at least in the context of current understanding of the temporal dynamics of plant diversification (Särkinen et al. 2013; Magallón et al. 2015; Mitchell et al. 2016). We therefore calibrated the root node with a lognormal calibration density parametrised as either: offset $=52, m=10.4, v=10.4$ (Calibration Strategy 2); or offset $=52, m=5.2, v=2.1$ (Calibration Strategy 3).

In Calibration Strategy 4, we calibrated the root node with a normal distribution $(\mu=$ 67.34, $\sigma=9.980)$. This is a secondary calibration from Magallón et al. (2015) in which 132 fossil calibrations were used throughout Spermatophyta. This calibration strategy is therefore not entirely dependent on the interpretation of fossil calibrations within Convolvulaceae and Solanaceae. 
We used RevBayes v1.0.4 (Hohna et al. 2014; Hohna, et al. 2016) to infer timecalibrated phylogenies according to the four calibration strategies. In all analyses, a GTR $+G$ + I model was used with a birth-death branching process as the tree prior. The topology was fixed to that inferred in MrBayes, and starting trees consistent with the 4 different calibration strategies were generated in treePL (Smith and O’Meara 2012). In one set of analyses, a strict clock was used where the substitution rate was assumed to be the same for every branch. In a second set of analyses, an uncorrelated lognormal (UCLN) relaxed clock (Drummond et al. 2006) was used. For each analysis, two independent runs were performed. Sufficient mixing and convergence was assessed in Tracer v1.6.0 (Rambaut et al. 2014). A 25\% burn-in was used prior to calculating maximum a posteriori (MAP) trees, mean posterior estimates (MPEs), and 95\% highest posterior density intervals (HPDs).

\subsection{Inferring the ages of major clades within Ipomoea}

We used the mean posterior estimate (MPE) for the age of Ipomoea from Calibration Strategy 1 as a point calibration for the root node of more densely sampled phylogenies for the genus. We used this MPE because the calibration strategy from which it is derived does not make any explicit assumptions about probable clade ages in relation to minimum or maximum constraints. It can therefore act as a basis from which to discuss the implications of calibration strategies that make explicit assumptions about probable clade ages in relation to minimum or maximum constraints.

Because we do not use further fossil calibrations within Ipomoea, we can easily interpret the implications of root node calibrations for Ipomoea derived from different calibration strategies - the relative ages of nodes within the time-calibrated phylogeny will not change, only the absolute timescale over which the time-calibrated phylogeny occurs. This also explains why we use a point calibration for the root node, rather than a calibration density. We can easily compare the effects of root node calibrations that derive from different calibration strategies. By contrast, it would be difficult to construct a single calibration density that adequately accounts for uncertainty in root node estimates that derive from each of the calibration strategies outlined above.

For divergence time analyses within Ipomoea, we were interested in estimating the ages of major clades from Muñoz-Rodríguez et al. (2019); and also the Parana Clade - a diverse clade centred in central South America; the Coriaceous Sepal Clade - a diverse clade centred in the Carribean; and the Batatas group - the clade of I. batatas and its closest wild relatives. We were also interested in constructing a temporal framework for subsequent analyses of diversification parameters. 
For these divergence time analyses, we used the molecular datasets and phylogenies from Muñoz-Rodríguez et al. 2019. This includes a nuclear dataset of 434 single copy genes for 211 species of Ipomoea, and a plastome dataset of 206 species of Ipomoea. Within these more densely-sampled datasets, there may be significant differences in diversification parameters among clades. This violates a key assumption of Bayesian methods of divergence time estimation that use a constant rate birth-death branching process as the tree prior. We therefore used treePL to infer divergence times - a penalised likelihood method that does not use a constant rate birth-death branching process (Smith and O'Meara 2012). In treePL, we performed analyses with the nuclear and chloroplast phylogenies from Muñoz-Rodríguez et al. (2019) as input trees. Following extensive cross-validation, we used a smoothing value of 0.01 for the nuclear phylogeny, and 10000 for the chloroplast phylogeny (lower smoothing values assume more among-branch-substitution-rate-variation than higher smoothing values). For both phylogenies, we also inferred time-calibrated phylogenies with alternative smoothing values (1, 100 and 10000 for nuclear, and 0.01, 1 and 100 for chloroplast). By experimenting with different smoothing values, we were able to determine the sensitivity of divergence time estimates to different assumptions about the magnitude of among-branchsubstitution-rate-variation.

Inferred divergence times within Ipomoea may be sensitive to the unique characteristics of different methods - especially given there are no calibrations within Ipomoea with which to constrain age estimates. We therefore also used RevBayes to estimate divergence times within Ipomoea in two sets of analyses.

In one set of analyses, we constructed skeletal time-calibrated phylogenies for Ipomoea with the taxon-sampling designed such that the ages of clades of interest (set out above) were estimated. For the nuclear data, we sampled all genes that were sampled in every taxon in the skeletal phylogeny (36 taxa) and for which the aligned sequence length was at least 4,000 sites. For the chloroplast data, we sampled the entire plastomes for 37 taxa.

Divergence time estimates in the skeletal time-calibrated phylogenies may be biased by the tree prior which assumes constant diversification parameters when inferring divergence times. In the second set of analyses performed in RevBayes, a series of three taxon time-calibrated phylogenies was therefore inferred, the divergence times within which would not be affected by the assumptions of the tree prior. The taxa sampled within these three-taxon phylogenies was such that the root node of Ipomoea was always sampled, along with one further node from within Ipomoea that uniquely corresponded to a node within the skeletal phylogenies. Thus, for each node in the skeletal phylogenies, we generated two age 
estimates - one derived from the skeletal phylogeny itself, and one from the three-taxon phylogeny that sampled that node. For three-taxon phylogenies constructed from nuclear data, we sampled all genes sampled for all three species in each three-taxon phylogeny. For the chloroplast data, we sampled the entire plastomes for the three species sampled in each three-taxon phylogeny.

In both the skeletal and three-taxon time-calibrated phylogenies, the MPE for the age of Ipomoea from Calibration Strategy 1 was used as a point calibration for the root node. In both sets of analyses, we also used the same alignments as Muñoz-Rodríguez et al. (2019). For the skeletal phylogenies, the total aligned sequence length was 39,495 sites for the nuclear data, and 103,921 sites for the chloroplast data. We used a GTR + G + I model of sequence evolution, and both a strict clock and a UCLN relaxed clock. A birth-death process was used as the tree prior for the skeletal phylogenies. A Yule process was used as the tree prior for the three-taxon phylogenies. The Yule process was parameterised such that the prior probability for the age of the internal node - the single unknown divergence time - was uniformly distributed between the root age and the present. Divergence time estimates from the three-taxon phylogenies are not therefore biased by the birth-death branching process. In all analyses, the topologies were fixed such that they were congruent with those inferred by Muñoz-Rodríguez et al. (2019). Two independent runs were performed. Sufficient mixing and convergence was assessed in Tracer v1.6.0. A $25 \%$ burn-in was used prior to calculating MAP trees, MPEs and 95\% HPDs.

\subsection{Inferring diversification parameters within Ipomoea}

We inferred diversification parameters in BAMM (Rabosky 2014). We used the 8 time-calibrated phylogenies inferred in treePL (smoothing values of $0.01,1,100,10000$ with nuclear and chloroplast phylogenies) as input trees. We could therefore evaluate how different assumptions about among-branch-substitution-rate-variation (and by extension differences in divergence time estimates), as well as topological differences between the nuclear and chloroplast phylogenies, affect inferred diversification parameters. Following consultation with John Wood, who has carried out the majority of the taxonomic work in this project, we specified approximate sampling proportions for different clades. We used the set_priors.R script - downloaded with BAMM and requiring the R package BAMMtools - to select the appropriate priors. Four independent runs were performed for each analysis. For the chloroplast time-calibrated phylogeny inferred with a smoothing value of 0.01 , the analysis was run for 4,000,000 generations. For the chloroplast time-calibrated phylogeny inferred with a smoothing value of 1 , and the nuclear time-calibrated phylogeny inferred with a 
smoothing value of 0.01 , the analyses were run for 2,000,000 generations. In all other cases, the analyses were run for 1,000,000 generations. A $10 \%$ burnin was used. Convergence was assessed using a custom R script that required the package BAMMtools. We compared Bayes factors between different models to identify the number of shifts in diversification parameters that occurred within our time-calibrated phylogenies. We then extracted the credible shift set - which represents the set of trees with different diversification parameter shift patterns that account for $95 \%$ of the posterior probability.

\subsection{When did the sweet potato evolve?}

To answer questions relating to the age of I. batatas, we used the MPE for the age of Ipomoea from Calibration Strategy 4 as a point calibration for the root node of timecalibrated phylogenies for Ipomoea. Calibration Strategy 4 does not account for the recently discovered and surprisingly old Physalis infinemundi. Further, several characteristics of the analysis (Magallón et al. 2015) from which Calibration Strategy 4 is derived are likely to mean that age estimates from this analysis are biased to younger ages. Age estimates derived from this calibration strategy are therefore likely to be considerably younger than those derived from Calibration Strategies 1 and 2 (and potentially 3). With respect to I. batatas, we are especially interested in determining whether its storage root evolved in pre-human times. Biasing our analysis to younger ages - by using Calibration Strategy 4 - is one of the most robust ways to do this, especially if, despite biasing analyses to younger ages, the recovered divergence times are pre-human.

We used this point calibration to calibrate the nuclear and chloroplast phylogenies for Ipomoea from Muñoz-Rodríguez et al. (2019). The time-calibrated phylogenies were inferred in treePL. The same optimum and alternative smoothing values were used as outlined previously.

The taxon sampling within these time-calibrated phylogenies is sufficient to estimate a divergence time between I. batatas and its closest wild relative I. trifida. Given I. trifida does not possess a storage root, the estimated divergence time between these two species can provide a basis for understanding when the storage root of $I$. batatas is likely to have evolved.

To scrutinise these divergence time estimates, we inferred additional time-calibrated phylogenies for the Batatas group in RevBayes. We could therefore explore the sensitivity of divergence time estimates in different methodological frameworks.

In an initial set of analyses in RevBayes, we used a point calibration for the root node (of the Batatas group) that was equal to the age estimate for the equivalent node in the 
nuclear time-calibrated phylogeny (for the entire genus) inferred with the optimum smoothing value in treePL. We inferred these phylogenies in RevBayes with both nuclear and chloroplast data from Muñoz-Rodríguez et al. (2018). For the nuclear data, we sampled all genes that were sampled in every species in the Batatas group. For the chloroplast data, we sampled the entire plastomes of every species in the Batatas group, except I. lactifera. The plastome of I. lactifera is highly reduced, and several problems resulted from its inclusion in preliminary analyses. We used the same alignments as Muñoz-Rodríguez et al. (2018). The total aligned sequence length was 375,628 sites for the nuclear data, and 160,435 sites for the chloroplast data. For both datasets, we used a GTR $+\mathrm{G}+$ I model of sequence evolution, and both a strict clock and a UCLN relaxed clock. We used a constant rate birth-death branching process as the tree prior. The topologies were fixed such that they were consistent with those inferred by Muñoz-Rodríguez et al. (2018). For each time-calibrated phylogeny, two independent runs were performed. Sufficient mixing and convergence was assessed in Tracer v1.6.0. A 25\% burn-in was used prior to calculating MAP trees, MPEs, and 95\% HPDs.

In a further set of analyses in RevBayes, the divergence between I. batatas and I. trifida was constrained such that it occurred $15 \mathrm{Ka}$, and was therefore in a timeframe that could allow for the storage root of I. batatas to be a product of human domestication. This constraint enabled us to infer the substitution rates required for the storage root of I. batatas to have evolved in human times. We performed two variants of this analysis. In the first, the root node of the Batatas group had the same point calibration as other analyses in this section. We therefore assume that the temporal framework inferred for the entire genus is broadly accurate. In the second, the root node did not have a point calibration. We therefore make no assumptions about the accuracy of the temporal framework inferred for the entire genus. This second variant is instead entirely independent of this temporal framework.

Where the root node had a point calibration, we expect our analysis may infer major differences in substitution rates between branches. As such we performed this analysis with both a UCLN relaxed clock - where substitution rates for each branch are drawn from a single continuous distribution, and a random local clock - where discrete (and potentially very large) changes in substitution rates between different branches are explicitly modelled (Drummond and Suchard 2011). Where the root node was not fixed, we used a strict clock. This additional set of analyses was performed with only the chloroplast data. As before, we used a GTR $+\mathrm{G}+\mathrm{I}$ model of sequence evolution and a birth-death branching process as the tree prior. The topologies were fixed such that they were consistent with those inferred by Muñoz-Rodríguez et al. (2018). For each time-calibrated phylogeny, two independent runs 
were performed. Sufficient mixing and convergence was assessed in Tracer v1.6.0. A 25\% burn-in was used prior to calculating MAP trees, MPEs, 95\% HPDs.

As well as estimating the divergence time between I. batatas and I. trifida, we also estimated the age of diversity within I. batatas. To do this, we inferred time-calibrated phylogenies with multiple accessions of I. batatas (71 accessions) and I. trifida (22 accessions). We inferred these time-calibrated phylogenies in treePL and RevBayes.

For analyses in treePL, we used nuclear and chloroplast phylogenies that contain multiple accessions of I. batatas and I. trifida (from Muñoz-Rodríguez et al. 2018) as input trees. We used a point calibration for the root node that was equal to the age estimate for the equivalent node in the nuclear time-calibrated phylogeny for the entire genus inferred with the optimum smoothing value in treePL. Following extensive cross-validation, we used a smoothing value of 0.01 for the nuclear and chloroplast phylogenies. We also inferred timecalibrated phylogenies with alternative smoothing values $(1,100,10000)$ to determine the sensitivity of divergence time estimates to different assumptions about the magnitude of among-branch-substitution-rate-variation.

In RevBayes, we performed two sets of analyses. First, we inferred time-calibrated phylogenies for all sampled accessions with either the nuclear or chloroplast data. For the nuclear data, we sampled the 40 genes that were sampled in every accession with the longest aligned sequence length. For the chloroplast data, we sampled entire plastomes. Second, we inferred a series of three-taxon time calibrated phylogenies with either the nuclear or chloroplast data. The motivation for this was the same as set out for the three-taxon timecalibrated phylogenies inferred across the whole of Ipomoea. The taxon sampling was such that in each three-taxon phylogeny, the root node of the phylogeny containing all sampled accessions was always sampled, along with one further node. For the nuclear data, we sampled all genes that were sampled for all three species in each three-taxon phylogeny. For the chloroplast data, we sampled the entire plastome for the three species sampled in each three-taxon phylogeny. We used the same alignments as Muñoz-Rodríguez et al. (2018).

In all these analyses, a GTR + G + I model of sequence evolution was used. We used either a strict clock or a UCLN relaxed clock. We used a point calibration for the root node that was equal to the age estimate for the equivalent node in the nuclear time-calibrated phylogeny for the entire genus inferred with the optimum smoothing value in treePL. For the time-calibrated phylogenies containing all sampled accessions, a birth-death branching process was used as the tree prior. For the three-taxon time-calibrated phylogenies, a Yule process was used as the tree prior. This was parameterised such that the prior probability for 
the age of the internal node - the single unknown divergence time - was uniformly distributed between the root age and the present. In all analyses, the topologies were fixed such that they were consistent with those inferred by Muñoz-Rodríguez et al. 2018. For each time-calibrated phylogeny, two independent runs were performed. Sufficient mixing and convergence was assessed in Tracer v1.6.0. A 25\% burn-in was used prior to calculating MAP trees, MPEs and 95\% HPDs.

\subsection{A comprehensive analysis of uncertainty in our understanding of the temporal dynamics of evolutionary diversification in Ipomoea}

The purpose of the large array of analyses presented here is to comprehensively evaluate and account for the different sources of uncertainty when inferring divergence times, and subsequently, diversification parameters. These different sources of uncertainty are highly interlinked and complex. In Figure 1, we summarise these different sources of uncertainty, and the ways in which we have accounted for them in this study.

\section{RESULTS}

\subsection{Time-calibrated phylogenies for Solanaceae and Convolvulaceae, and an age estimate for Ipomoea}

Here, we summarise results when a UCLN relaxed clock is used with different calibration strategies for Convolvulaceae and Solanaceae, followed by results when using a strict clock.

Calibration Strategy 1 resulted in the oldest divergence time estimates throughout Convolvulaceae and Solanaceae (Fig. 2a). The 95\% HPD for the age of the divergence between Convolvulaceae and Solanaceae was 97-130 Myr, the 95\% HPD for the age of the Convolvulaceae crown node was 94-129 Myr, the 95\% HPD for the age of the Solanaceae crown node was 67-99 Myr, and the 95\% HPD for the age of the Ipomoea crown node was 18-43 Myr. There was a 20.3-fold difference between the maximum inferred substitution rate (MPE) of $2.9^{*} 10^{-3}$ substitutions $\mathrm{Myr}^{-1}$ (on the Solanaceae stem branch), and the minimum inferred substitution rate of $1.4 * 10^{-4}$ substitutions $\mathrm{Myr}^{-1}$ (Fig. 2a and S1a). However, apart from the Solanaceae stem branch, inferred rates are within a range between $1.4^{*} 10^{-4}$ and $1.4 * 10^{-3}$ substitutions $\mathrm{Myr}^{-1}$, a 10-fold difference.

Calibration Strategy 2 resulted in younger divergence time estimates (Fig. 2b). The 95\% HPD for the age of the divergence between Convolvulaceae and Solanaceae was 89-129 Myr, the 95\% HPD for the age of the Convolvulaceae crown node was 86-126 Myr, the 95\% HPD for the age of the Solanaceae crown node was 64-94 Myr, and the 95\% HPD for the age of the Ipomoea crown node was 17-41 Myr. There was a 21.5-fold difference between the 
maximum inferred substitution rate of $3.2^{*} 10^{-3}$ substitutions $\mathrm{Myr}^{-1}$ (on the Solanaceae stem branch), and the minimum inferred substitution rate of $1.5^{*} 10^{-4}$ substitutions $\mathrm{Myr}^{-1}$ (Fig. $2 \mathrm{~b}$ and S1b). However, apart from the Solanaceae stem branch, inferred rates fall within a range between $1.5^{*} 10^{-4}$ and $1.5^{*} 10^{-3}$ substitutions $\mathrm{Myr}^{-1}$, a 10-fold difference.

Calibration Strategy 3 resulted in the youngest divergence time estimates (Fig. 2). The 95\% HPD for the age of the divergence between Convolvulaceae and Solanaceae was 57-58 Myr, the 95\% HPD for the age of the Convolvulaceae crown node was 46-56 Myr, the 95\% HPD for the age of the Solanaceae crown node was 56-58 Myr, and the 95\% HPD for the age of the Ipomoea crown node was 10-21 Myr. There was a 59-fold difference between the maximum inferred substitution rate of $1.3^{*} 10^{-2}$ substitutions $\mathrm{Myr}^{-1}$ (on the Convolvulaceae stem branch), and the minimum inferred substitution rate of $2.1 * 10^{-4}$ substitutions $\mathrm{Myr}^{-1}$ (Fig. 2c and S1c). However, apart from the Convolvulaceae stem branch, inferred rates fall within a range between $1.5^{*} 10^{-4}$ and $3.5^{*} 10^{-3}$ substitutions $\mathrm{Myr}^{-1}$, a 16-fold difference.

Calibration Strategy 4 also resulted in younger divergence time estimates than Calibration Strategy 1 and 2 (Fig. 2). The 95\% HPD for the age of the divergence between Convolvulaceae and Solanaceae was 41-83 Myr, the 95\% HPD for the age of the Convolvulaceae crown node was 40-81 Myr, the 95\% HPD for the age of the Solanaceae crown node was 26-59 Myr, and the 95\% HPD for the age of the Ipomoea crown node was 8$25 \mathrm{Ma}$. There was a 17.9-fold difference between the maximum inferred substitution rate of $4.8^{*} 10^{-3}$ substitutions $\mathrm{Myr}^{-1}$ (on the Solanaceae stem branch), and the minimum inferred substitution rate of $2.7 * 10^{-4}$ substitutions $\mathrm{Myr}^{-1}$ (Fig. $2 \mathrm{~d}$ and S1d). However, apart from the Solanaceae stem branch, inferred rates fall within a range between $1.5^{*} 10^{-4}$ and $2.5^{*} 10^{-3}$ substitutions $\mathrm{Myr}^{-1}$, a 9.2-fold difference.

With a strict clock, Calibration Strategies 1-3 resulted in divergence time estimates that were very similar to each other, and to divergence time estimates with Calibration Strategy 1 and a UCLN relaxed clock (Fig. S2a-c; Fig. 2a). Inferred substitution rates were also very similar for Calibration Strategies 1-3 with a strict clock (Fig. S2a-c). With a strict clock, Calibration Strategy 4 resulted in markedly younger divergence time estimates than Calibration Strategies 1-3 with a strict clock and calibration Strategy 4 with a UCLN relaxed clock (Fig. S2d, Fig. 2d). For all four calibration strategies, 95\% HPDs were far narrower with a strict clock (Fig. 2, Fig. S2).

\subsection{Divergence time estimates within Ipomoea}

\subsubsection{Divergence time estimates within Ipomoea inferred in treePL with the optimum} smoothing value 
When the optimum smoothing value of 0.01 was used to infer a nuclear timecalibrated phylogeny in treePL, the inferred crown ages for the New World and Old World Clades were approximately 29 Myr and 28 Myr respectively (Fig. 3a, Fig. S3a). The major clades A-E, and Astripomoea and close relatives, are inferred to have originated 16-23 Ma, throughout the Early Miocene (Fig 3a, Fig. S3a). The Parana Clade and Coriaceous Sepal Clade, and the Batatas group are inferred to have originated 7.5-12.5 Ma, during the Late Miocene. In this time-calibrated phylogeny, there was a 3.279-fold difference between the minimum inferred substitution rate of $6.21 * 10^{-4}$ substitutions $\mathrm{Myr}^{-1}$, and the maximum inferred substitution rate of $2.036^{*} 10^{-3}$ substitutions $\mathrm{Myr}^{-1}$ (Fig. 3a, Fig. S4a).

When the optimum smoothing value of 10000 was used to infer a chloroplast timecalibrated phylogeny in treePL, the inferred crown ages for the New World and Old World Clades were approximately 25.6 Myr and 21 Myr respectively (Fig. 3b, Fig. S3b). Clades A, B, D, E, and Astripomoea and close relatives, are inferred to have originated 13.5-19.1 Ma, throughout the Early and Middle Miocene. Clade $\mathrm{C}$ is inferred to have originated during the Late Miocene, $8.9 \mathrm{Ma}$ (Fig. 3b, Fig. S3b). In this time-calibrated phylogeny, the same substitution rate of $1.81 * 10^{-4}$ substitutions $\mathrm{Myr}^{-1}$ was inferred for every branch (Fig. 3b).

These results from the nuclear and chloroplast time-calibrated phylogenies indicate that much of the morphological and species diversity within Ipomoea, briefly summarised in Figure $3 \mathrm{c}-\mathrm{j}$, originated before the end of the Miocene. However, the limited taxon sampling means that it is difficult to construct detailed or precise hypotheses about when and how frequently specific morphological traits evolved.

\subsubsection{Alternative divergence time estimates for the Ipomoea nuclear phylogeny}

Divergence times inferred in treePL with alternative smoothing values were similar to divergence times inferred with the optimum value, although in some cases they were somewhat younger (Fig. S3a, Fig. S5a-c, Table 1). With higher smoothing values, the magnitude of among-branch-substitution-rate-variation decreased (Fig. S4a-c, Fig. S5a-c).

For analyses in RevBayes, in the skeletal time-calibrated phylogeny inferred with a strict clock, MPEs for the ages of major clades were similar to age estimates inferred in treePL (Fig. S3a, Fig. S6a, Table 1). 95\% HPD intervals were on average 10.9\% of the MPE for the age each clade (Fig. S3a, Table 1), and the inferred substitution rate was similar to those inferred in treePL (Fig. S6a). In the three-taxon time-calibrated phylogenies inferred with a strict clock, MPEs for the ages of major clades were somewhat older than in the skeletal time-calibrated phylogeny and 95\% HPDs were on average $4.7 \%$ of the MPE for the age of each clade (Fig. S3a, Table 1). 
In the skeletal time-calibrated phylogenies inferred with a relaxed clock, MPEs for the ages of major clades were similar to age estimates inferred in treePL and to the skeletal timecalibrated phylogeny inferred with a strict clock. However, there was more variation in the relationship between these age estimates and other estimates, compared to those previously discussed, with inferred ages being either older or younger depending on the clade (Fig. S3a, Fig. S6a, Table 1). 95\% HPD intervals were markedly wider than in RevBayes analyses with a strict clock, on average they were $60 \%$ of the MPE for the age of each clade (Fig. S3a, Table 1). The substitution rate and magnitude of among-branch-substitution-rate-variation was similar to that inferred in treePL with a lower smoothing value (Fig. S6b, Fig. S7a). In three-taxon time-calibrated phylogenies inferred with a relaxed clock, MPEs tended to be slightly older than MPEs inferred in either of the skeletal time-calibrated phylogenies. However, for the two oldest clades in the phylogeny, the New Word and Old World clades, MPEs were markedly younger than other age estimates inferred in treePL or RevBayes (Fig. S3a, Table 1). 95\% HPD intervals were considerably wider compared to the skeletal timecalibrated phylogeny inferred with a relaxed clock. On average they were $114.1 \%$ of the MPE for the age of each clade (Fig. S3a, Table 1).

\subsubsection{Alternative divergence time estimates for the Ipomoea chloroplast phylogeny}

Divergence times inferred in treePL with a smoothing value of 100 were very similar to divergence times inferred with a smoothing value of 10000 (Fig. S3b, Fig. S5f, Table 1). With a smoothing value of 0.01 or 1 , inferred divergence times were significantly older. This was especially the case for clades A-E, the majority of which were inferred to have originated during the earliest Miocene or Late Oligocene (Fig. S2b, Fig. S5d and e, Table 1). With lower smoothing values, the magnitude of among-branch-substitution-rate-variation increased (Fig. S4d-f, Fig S5d-f). With the lowest smoothing value of 0.01, there was a 193-fold difference between the maximum inferred substitution rate of $3.86^{*} 10^{-4}$ substitutions $\mathrm{Myr}^{-1}$, and the minimum inferred substitution rate of $2 * 10^{-6}$ substitutions $\mathrm{Myr}^{-1}$.

For analyses in RevBayes, in skeletal time-calibrated phylogenies inferred with a strict clock, MPEs for the ages of major clades were most similar to age estimates inferred in treePL with a smoothing value of 10000 (Fig. S3b, Fig. S6c, Table 1). 95\% HPD intervals were approximately $16 \%$ of the MPE for the age of each clade (Fig. S3b, Table 1). As with divergence times, the inferred substitution rate was also similar to when a smoothing value of 10000 was used in treePL (Fig. S6c). In three-taxon time calibrated phylogenies inferred with a strict clock, MPEs for the ages of major clades were similar to those inferred in the skeletal time-calibrated phylogeny (Fig. S3b, Table 1). 95\% HPDs were slightly wider than in the 
skeletal time-calibrated phylogeny, approximately $19 \%$ of the MPE for each clade (Fig. S3b, Table 1).

In skeletal time-calibrated phylogenies inferred with a relaxed clock, MPEs for the ages of major clades tended to be slightly older than in either of the two types of timecalibrated phylogeny inferred with a strict clock (Fig. S3b, Fig. S6d, Table 1). They were therefore also slightly closer to age estimates inferred in treePL with smoothing values of 0.01 and $1.95 \%$ HPDs were also markedly wider than RevBayes analyses with a strict clock, approximately $72 \%$ of the MPE for the age of each clade (Fig. S3b, Table 1). The substitution rate, and magnitude of among-branch-substitution-rate-variation was similar to the chloroplast time-calibrated phylogeny inferred in treePL with a smoothing value of 0.01 (Fig. S6d, Fig. S7b). In three-taxon time-calibrated phylogenies inferred with a relaxed clock, MPEs for the ages of major clades tended to be slightly older than MPEs inferred according to alternative methods in RevBayes (Fig. S3b, Table 1). 95\% HPD intervals were also markedly wider, approximately $132.4 \%$ of the MPE for each clade (Fig. S3b, Table 1).

Table 1. Results summary of time-calibrated phylogenies for Ipomoea

\begin{tabular}{ccc}
\hline Inference Method & $\begin{array}{c}\text { \% Change in age } \\
\text { estimate relative } \\
\text { treePL with } \\
\text { smoothing }=\mathbf{0 . 0 1}\end{array}$ & $\begin{array}{c}\text { Mean 95\% HPD } \\
\text { width }\end{array}$ \\
\hline Nuclear, smoothing = 0.01 & -- & -- \\
\hline Nuclear, smoothing = 1 & -10.1 & -- \\
\hline Nuclear, smoothing = 100 & -10.3 & -- \\
\hline Nuclear, smoothing = 10000 & -10.3 & -- \\
\hline Nuclear, skeletal strict clock & -15.3 & 10.9 \\
\hline Nuclear, three-taxon strict clock & 7.2 & 4.7 \\
\hline Nuclear, skeletal UCLN relaxed clock & -12.3 & 60.0 \\
\hline Nuclear, three-taxon UCLN relaxed clock & 7.0 & 114.1 \\
\hline Chloroplast, smoothing = 0.01 & -- & -- \\
\hline Chloroplast, smoothing = 1 & -- \\
\hline Chloroplast, smoothing = 100 & -2.1 & -- \\
\hline Chloroplast, smoothing $=\mathbf{1 0 0 0 0}$ & -27.3 & 15.9 \\
\hline Chloroplast, skeletal strict clock & -27.6 & 18.9 \\
\hline Chloroplast, three-taxon strict clock & -26.8 & 71.5 \\
\hline Chloroplast, skeletal UCLN relaxed clock & -23.6 & 132.4 \\
\hline Chloroplast, three-taxon UCLN relaxed clock & -22.3 & - \\
\hline
\end{tabular}

\subsection{Diversification parameter estimates within Ipomoea}

\subsubsection{Nuclear Phylogeny}

Inferred diversification parameters were similar, regardless of the smoothing value that was used to infer the time-calibrated phylogeny (Fig. 3a. Fig. S5a-c, Table 2). With the time-calibrated phylogeny inferred with the optimum smoothing value of 0.01 , a single shift 
in diversification parameters near the origin of a clade that contains the Parana Clade and Coriaceous Sepal Clade, has by far the highest posterior probability $(p=0.9)$ (Fig. 3a). This diversification parameter shift leads to a 5.9-fold increase in the net diversification rate to 0.802 species $\mathrm{Myr}^{-1}$, compared to the background rate of 0.136 species $\mathrm{Myr}^{-1}$. This net diversification rate increase is caused by an increase in the inferred speciation rate from 0.147 , to 0.820 species $\mathrm{Myr}^{-1}$ (Table 2).

\subsubsection{Chloroplast Phylogeny}

In the time-calibrated phylogeny inferred with the optimum smoothing value of 10000, a single shift in diversification parameters near the origin of the Parana Clade has the highest posterior probability ( $p=0.79$ ) (Fig. 3b). Two alternative patterns, in which there are shifts in diversification parameters on immediately ancestral branches, have significantly lower posterior probabilities (Fig. 3b). The most probable diversification parameter shift is inferred to lead to a 3.2-fold increase in net the diversification rate to 0.641 species $\mathrm{Myr}^{-1}$, compared to the background rate of 0.199 species $\mathrm{Myr}^{-1}$ (Table 2). This net diversification rate increase is caused by an increase in the speciation rate from 0.212 to 0.768 species $\mathrm{Myr}^{-1}$. With the time-calibrated phylogeny inferred with a smoothing value of 100 , inferred diversification parameters were very similar to when a smoothing value of 10000 was used (Fig. S5f, Table 2). With the time-calibrated phylogenies inferred with smoothing values of 1 and 0.01, no discrete shifts in diversification parameters were inferred (Fig. S5d and e, Table 2).

Table 2. Diversification parameter estimates for Ipomoea inferred in BAMM using different time-calibrated phylogenies as input trees.

\begin{tabular}{|c|c|c|c|c|}
\hline Time-calibrated phylogeny & $\begin{array}{c}\text { Background } \\
\text { speciation rate } \\
\left(\text { species } \mathrm{Myr}^{-1}\right)\end{array}$ & $\begin{array}{c}\text { Background } \\
\text { extinction rate } \\
\left(\text { species } \mathrm{Myr}^{-1}\right)\end{array}$ & $\begin{array}{c}\text { Shift } \\
\text { speciation } \\
\text { rate* } \\
\left(\begin{array}{c}\text { species Myr } \\
\text { 1) }\end{array}\right. \\
\end{array}$ & $\begin{array}{c}\text { Shift } \\
\text { extinction } \\
\text { rate* } \\
\left(\text { species }^{*}\right. \\
\left.\text { Myr }^{-1}\right) \\
\end{array}$ \\
\hline Nuclear, smoothing $=0.01$ & 0.147 & 0.0108 & 0.820 & 0.0176 \\
\hline Nuclear, smoothing = 1 & 0.150 & 0.00942 & 0.878 & 0.0184 \\
\hline Nuclear, smoothing $=100$ & 0.150 & 0.00950 & 0.895 & 0.0182 \\
\hline Nuclear, smoothing $=10000$ & 0.150 & 0.00954 & 0.898 & 0.0190 \\
\hline Chloroplast, smoothing $=0.01$ & 0.153 & 0.0136 & --- & --- \\
\hline Chloroplast, smoothing = 1 & 0.150 & 0.0123 & --- & --- \\
\hline Chloroplast, smoothing $=\mathbf{1 0 0}$ & 0.212 & 0.0135 & 0.775 & 0.131 \\
\hline Chloroplast, smoothing $=10000$ & 0.212 & 0.0131 & 0.768 & 0.126 \\
\hline
\end{tabular}

\subsection{When did the sweet potato evolve?}




\subsubsection{When did the sweet potato diverge from its closest relative?}

In time-calibrated phylogenies for the Batatas group inferred in RevBayes with a UCLN relaxed clock, the divergence between I. batatas and I. trifida was inferred to have occurred at least 1.9Ma in the nuclear time-calibrated phylogeny (Fig. 4a), and at least $0.89 \mathrm{Ma}$ in the chloroplast time-calibrated phylogeny (Fig. 4b). In nuclear and chloroplast time-calibrated phylogenies inferred with alternative methodologies, the inferred divergence time between I. batatas and I. trifida was always inferred to have occurred at least $0.8 \mathrm{Ma}$ (Fig. S8, Fig. S9). These alternative methodologies make different assumptions about the magnitude and nature of among-branch-substitution-rate-variation. As such, each analysis makes different inferences about substitution rates on individual branches (Fig. 4, Fig. S8, and Fig. S9). Regardless of this, in all cases the divergence between I. batatas and I. trifida is inferred to have occurred in pre-human times.

We performed two further analyses with our chloroplast dataset. Where we constrained the divergence between I. batatas and I. trifida to have occurred no more than 15 $\mathrm{Ka}$, but retained the point calibration of 6.2 Myr at the root node - we inferred a greater than 200-fold increase in substitution rates for branches leading to I. batatas and I. trifida. As a result, substitution rates in this part of the time-calibrated phylogeny exceed $2.2^{*} 10^{-2}$ substitutions Myr $^{-1}$ (Fig. 5a, Fig. S10). This value far exceeds any previously inferred substitution or mutation rate for chloroplast DNA. This result was consistent regardless of whether we used a UCLN relaxed clock (Fig. 5a) or a random local clock (Fig. S10).

Where we constrained the divergence between I. batatas and I. trifida to occur $15 \mathrm{Ka}$, but removed the point calibration at the root node, we inferred a substitution rate of $2.1 * 10^{-2}$ substitutions $\mathrm{Myr}^{-1}$ for the Batatas group (Fig. 5b). As before, this rate is far higher than any previously inferred substitution or mutation rate for chloroplast DNA. Taken together, these two additional analyses demonstrate that in order to infer a divergence time between $I$. batatas and I. trifida that occurred in human times, and that is consistent with the storage root of sweet potato having originated as a result of human domestication, a substitution rate would be required that far exceeds any known substitution or mutation rate for chloroplast DNA.

\subsubsection{When did diversity within the sweet potato evolve?}

With the time-calibrated phylogenies that contained all sampled accessions of $I$. batatas and I. trifida, the divergences between most of the lineages within I. batatas occurred in pre-human times (Fig. 6). We interpret this as strong evidence that most of the diversity within I. batatas evolved in pre-human times. This result was consistent with both the nuclear 
(Fig. 6a) and chloroplast datasets (Fig. 6b), and for analyses inferred in treePL with both the optimum and alternative smoothing values, or in RevBayes with a strict clock or UCLN relaxed clock (Fig. S11, Fig. S12). These alternative methodologies make different assumptions about the magnitude and nature of among-branch-substitution-rate-variation. As such, each analysis makes different inferences about substitution rates on individual branches (Fig. 6, Fig. S12-14). Regardless of this, in all cases the divergences between different accessions of I. batatas and I. trifida are inferred to have occurred in pre-human times.

In RevBayes, we also inferred divergence times in three-taxon time-calibrated phylogenies. The divergence time estimates in these three-taxon time-calibrated phylogenies differed somewhat from those in which all taxa were sampled and HPDs were considerably wider (Table 3, Fig. S14). However, as before, the divergences between different accessions of I. batatas and I. trifida predominantly occurred in pre-human times.

Table 2. Results summary of time-calibrated phylogenies inferred in RevBayes for estimating the age of diversity within I. batatas and I. trifida

\begin{tabular}{|c|c|c|c|c|c|}
\hline & $\begin{array}{l}\text { \% change in MPE } \\
\text { in three-taxon } \\
\text { time-calibrated } \\
\text { phylogeny } \\
\text { compared to time- } \\
\text { calibrated- } \\
\text { phylogeny with all } \\
\text { specimens } \\
\end{array}$ & $\begin{array}{c}\text { Mean 95\% } \\
\text { HPD } \\
\text { width (as } \\
\% \text { of } \\
\text { MPE) - all } \\
\text { specimens }\end{array}$ & $\begin{array}{l}\text { Mean 95\% } \\
\text { HPD width } \\
\text { (as \% of } \\
\text { MPE) - } \\
\text { three taxon }\end{array}$ & $\begin{array}{l}\% \text { of nodes with } \\
95 \% \text { HPD that } \\
\text { includes human } \\
\text { era }(<15 \mathrm{Ka})-\text { all } \\
\text { specimens }\end{array}$ & $\begin{array}{c}\% \text { of nodes } \\
\text { with } 95 \% \\
\text { HPD that } \\
\text { includes } \\
\text { human era (< } \\
15 \text { Ka) - } \\
\text { three taxon }\end{array}$ \\
\hline $\begin{array}{c}\text { Nuclear, strict } \\
\text { clock }\end{array}$ & 29.0 & 13.5 & 12.7 & 0 & 1.5 \\
\hline $\begin{array}{l}\text { Nuclear, UCLN } \\
\text { relaxed clock }\end{array}$ & -16.0 & 41.6 & 124.3 & 0 & 0 \\
\hline $\begin{array}{l}\text { Chloroplast, } \\
\text { Strict clock }\end{array}$ & 33.9 & 65.4 & 94.0 & 0 & 5.9 \\
\hline $\begin{array}{c}\text { Chloroplast, } \\
\text { UCLN relaxed } \\
\text { clock }\end{array}$ & 33.5 & 111.1 & 183.7 & 1.5 & 2.9 \\
\hline
\end{tabular}

\section{DISCUSSION}

\subsection{Calibration uncertainty provides an uncertain basis for inferring the temporal}

\section{dynamics evolution in Ipomoea}

Different combinations and interpretations of fossil calibrations provide an uncertain and conflicting framework for calibrating the phylogeny of Convolvulaceae and Solanaceae. As such, different calibration strategies led to major differences in inferred divergence times within the two families, including for the crown node of Ipomoea (Fig. 2). With respect to fossils within Solanaceae, this study focussed on the recently discovered Physalis infinemundi, and the implications of different assumptions about the maximum possible age 
of Solanaceae. We showed that making stronger assumptions that the Solanaceae stem node is significantly younger than $130 \mathrm{Myr}$ - a commonly used hard maximum for the origin of Eudicots - led to significantly younger divergence time estimates. This was the case even when Physalis infinemundi was retained as a minimum constraint of $52 \mathrm{Myr}$ for the Solanoideae stem node. In Calibration Strategy 3, where we made the strongest assumption that the Solanaceae stem node was significantly younger than $130 \mathrm{Myr}$, age estimates were similar to those obtained in previous studies where significantly younger fossil calibrations were used (Särkinen et al. 2013; Magallón et al. 2015; Mitchell et al. 2016). As well as evaluating fossil calibrations within Solanaceae, we also constructed a time-calibrated phylogeny with a secondary calibration from Magallón et al. (2015). This led to younger divergence time estimates that were similar to Calibration Strategy 3.

It is impossible to make a definitive judgement about which calibration strategy is the most robust. Assuming its phylogenetic placement is correct, Physalis infinemundi does provide a minimum constraint for the Solanoideae stem node. However, it would be misleading to suggest we know a most probable age for the Solanoideae stem node in relation to the age of this fossil. Alternatively, it may be reasonable to suggest that the Solanaceae stem node must be younger than 130Myr - but the likely extent to which it is younger is also difficult to quantify. Further, the maximum constraint of 130Myr is derived from the observation that there is an absence of Eudicot fossils in sediments older than this age, but a sudden appearance in younger sediments (Doyle et al. 1977). This maximum constraint is therefore based on the absence of evidence - a problematic basis upon which to construct assumptions.

The implications of different calibration strategies are also influenced by assumptions about among-branch-substitution-rate-variation. Note, for example, how the same calibration strategy can lead to significantly different divergence time estimates, depending on whether a UCLN relaxed clock or a strict clock is used (Fig. 2 and Fig. S2). Further, for the same molecular clock model, different calibration strategies lead to marked differences in inferred substitution rates (Fig. 2, Fig. S1, Fig. S2). Taken together, this highlights the tight interaction between fossil calibrations, assumptions about among-branch-substitution-ratevariation, inferred substitution rates, and inferred divergence times.

Despite the uncertainty presented here, we suggest that presenting our results in this way provides the most robust basis for interpreting divergence time estimates, and accounting for factors that may affect inferences in the future. We do not, by contrast, present a single time-calibrated phylogeny, because no single time-calibrated phylogeny fully accounts for all 
the uncertainty that is represented here. Despite this complexity, with careful interpretation we suggest these results can provide a basis for discussing the temporal dynamics of evolution in Ipomoea, and answering specific questions about the origin of I. batatas.

\subsection{Uncertainty about substitution rate variation within Ipomoea leads to further uncertainty in divergence time estimates}

We did not implement any fossil calibrations at internal nodes within Ipomoea. Therefore, whilst uncertainty about the absolute age of the entire of Ipomoea can result from calibration uncertainty (summarised in Figure 2), uncertainty about the relative ages of different clades within Ipomoea cannot result from calibration uncertainty. Instead, it results predominantly from assumptions about substitution rates, and in some cases additional assumptions about relative clade ages (expressed by the birth-death branching process in Bayesian analyses). In our analyses of divergence times within Ipomoea, we therefore experimented with different assumptions about substitution rates, and in Bayesian analyses, with the assumptions of the birth-death branching process.

In nuclear time-calibrated phylogenies inferred in treePL, we showed that different smoothing values have a limited effect on inferred divergence times (Fig. 3a, Fig S3a, Fig. S5a-c, Table 1). Regardless of the smoothing value, the majority of major clades are inferred to have originated in the Late Oligocene and throughout the Early Miocene, and the Parana Clade, Coriaceous Sepal Clade, and the Batatas group are inferred to have originated in the Late Miocene. This implies that inferred divergence times within Ipomoea are fairly robust to assumptions about among-branch-substitution-rate-variation. This result is likely to be explained by the fact that regardless of the smoothing value, the magnitude of inferred among-branch-substitution-rate-variation was always fairly low (Fig. 3a, Fig. S4a-c, Fig. S5a-c). Nonetheless, any conclusion about the robustness of these divergence time estimates assumes that the model of rate variation used in treePL - whereby substitution rates are correlated between ancestral and descendant branches (Sanderson 1997; Smith and O’Meara 2012) - is valid.

By contrast, in chloroplast time-calibrated phylogenies inferred in treePL, different smoothing values had a more profound effect on inferred divergence times. Specifically, lower smoothing values ( 0.01 and 1$)$ caused basal branches to be considerably shorter - and by consequence have considerably higher substitution rates - compared to higher smoothing values. This in turn meant that with lower smoothing values, inferred divergence times were considerably older such that the majority of major clades originated in the Late Oligocene or very early Miocene (Fig. 3b, Fig. S3b, Fig. S4d-f, Fig. S5d-f, Table 1). Clearly, this result 
highlights that there is considerable uncertainty in divergence time estimates within Ipomoea. However, when experimenting with different smoothing values, we performed extensive cross-validation analyses to determine which smoothing values are most appropriate. For the chloroplast data, these suggested that higher smoothing values are more appropriate. Further, we suggest that divergence times inferred with higher smoothing values are more congruent with the nuclear time-calibrated phylogeny, with major clades originating throughout the Early Miocene, rather than exclusively in the very early Miocene. We therefore tentatively suggest that divergence times inferred with higher smoothing values are more accurate (Fig. $3 b)$.

For analyses performed with nuclear data in RevBayes, the skeletal and three-taxon time-calibrated phylogenies were broadly congruent with those inferred in treePL (Fig. S3a, Fig. S6a and b, Table 1). This provides further evidence that divergence time estimates with this dataset are relatively insensitive to assumptions about among-branch-substitution-ratevariation. However, we note that 95\% HPDs are considerably wider when a UCLN relaxed clock is used compared to a strict clock (Fig. S3a, Table 1). This highlights how uncertainty about substitution rates for individual branches - a source of uncertainty that is explicitly accounted for with a UCLN relaxed clock but not with a strict clock - leads to uncertainty in divergence time estimates. We also note that when a UCLN relaxed clock is used, 95\% HPD widths are considerably wider in three-taxon time calibrated phylogenies compared to skeletal time-calibrated phylogenies (Fig. S3a, Table 1). We suggest this reflects the fact that in the skeletal time-calibrated phylogenies, the birth-death branching process - which makes assumptions about divergence times - causes greater precision in divergence time estimates. This contrasts to the three-taxon time-calibrated phylogenies, where the birth-death branching process does make any assumptions about divergence times. This result has concerning implications because it suggests that the precision of divergence time estimates is sensitive to the assumption of the birth-death branching process that diversification parameters are constant. Given that this assumption is often likely to be violated, this precision may be misleading. Nonetheless, we tentatively suggest that the uncertainty expressed in the threetaxon time-calibrated phylogenies may be excessive given that all the time-calibrated phylogenies inferred in treePL corroborate with each other, and with the skeletal timecalibrated phylogenies inferred in RevBayes. However, we make this assertion whilst also considering the potential sources of error outlined above.

For analyses performed with chloroplast data in RevBayes, the skeletal and threetaxon time-calibrated phylogenies were broadly congruent with those inferred in treePL with 
smoothing values of 100 and 10000 (Fig. S3b, Fig. S6c and d, Table 1). Given that smoothing values of 100 and 10000 are likely to be more appropriate for this dataset, and corroborate inferences derived from nuclear data, this result supports our previous inferences about when major clades within Ipomoea evolved (Fig. 3). Like with the nuclear data, 95\% HPDs were considerably wider when a UCLN relaxed clock was used, and when divergence times were inferred in three-taxon time-calibrated phylogenies. We suggest the same explanations as in the discussion of nuclear time-calibrated phylogenies.

Taken together, these results provide tentative support that the majority of major clades within Ipomoea evolved in the Late Oligocene and throughout the Early Miocene, and the Parana Clade, Coriaceous Sepal Clade, and the Batatas group evolved in the Late Miocene. However, these conclusions are dependent on several important variables. These include the Calibration Strategy used to infer the time-calibrated phylogeny for Convolvulaceae and Solanaceae, the assumptions of different methods that account for among-branch-substitution-rate-variation, and - in Bayesian analyses - the assumption of a constant rate of speciation and extinction. It is important to consider the effect of these variables when interpreting the divergence time estimates presented here. Specifically, the calibration strategy will affect inferences of the absolute age of the entire of Ipomoea, whilst assumptions about the magnitude and nature of among-branch-substitution-rate-variation and the constancy of speciation and extinction rates, will affect inferences of the relative ages of different clades.

\subsection{Significant diversification parameter variation within Ipomoea is highly likely}

Inferences of diversification parameters are sensitive to uncertainty and error in divergence time estimates. In order to account for this, we inferred diversification parameters on time-calibrated phylogenies inferred with different smoothing values in treePL. With the nuclear time-calibrated phylogenies, we inferred a significant net diversification rate increase for the clade that contains both the Parana Clade and Coriaceous Sepal Clade. This net diversification rate increase was strongly supported, regardless of the smoothing value (Fig. 3a, Fig. S5a-c, Table 2). We were not surprised to infer a net diversification rate increase for this clade. Species within the Parana Clade and Coriaceous Sepal Clade often have very localised geographical ranges, contain groups of species whose morphologies overlap significantly, but overall, exhibit a variety of different growth forms and occur in a range of different habitats. These are characteristic attributes of rapid evolutionary radiations (Hughes and Eastwood. 2006; Givnish et al. 2009; Nevado et al. 2016). 
With the chloroplast time-calibrated phylogenies inferred with smoothing values of 100 and 10000, we inferred a significant net diversification rate increase in the Parana Clade but not the Coriaceous Sepal Clade. By contrast, we did not infer any significant shifts in diversification parameters when a smoothing value of 0.01 or 1 was used (Fig. 3b, Fig. S5d-f, Table 2). However, given our cross-validation analyses suggest smoothing values of 100 and 10000 are more appropriate, we suggest the chloroplast dataset supports a significant net diversification rate increase at the origin of the Parana Clade. Taken together, the nuclear and chloroplast time-calibrated phylogenies provide strong support for a significant net diversification rate increase at either the origin of the clade containing the Parana Clade and Coriaceous Sepal Clade, or at the origin of the Parana Clade.

Despite the likely presence of net diversification rate shifts within Ipomoea, there is considerable uncertainty about the nature of these shifts. First, the possibility of erroneous divergence time estimates means there is considerable uncertainty about when the net diversification rate shifts actually occurred. Although we accounted for divergence time estimation error to an extent, by inferring diversification parameters on time-calibrated phylogenies inferred with different smoothing values, we did not account for other factors. For example, calibration uncertainty may mean that the entire phylogeny is calibrated to an incorrect timescale. A further factor that will likely affect the inferred timing and number of net diversification rate shifts, is the limited sampling within the phylogeny. For example, in the Parana Clade, only around $33 \%$ of the species are sampled. Although we corrected for this in BAMM by specifying sampling proportions, a robust and well-resolved phylogeny that includes these missing species may result in the inference of several nested net diversification rate shifts that could not be recovered in the analyses presented here.

A further aspect of these net diversification rate shifts for which considerable uncertainty remains, is whether they reflect changes in the speciation rate or extinction rate. Our analyses in BAMM suggest they result from significant increases in the speciation rate (Table 2). However, in many cases it is likely to be very difficult to determine the relative contribution of speciation rate changes and extinction rate changes on inferred diversification dynamics (Rabosky 2010, 2014; Sanderson and Donoghue 2015; Moore et al. 2016). This is primarily because extinct branches are not sampled in a molecular phylogeny. Uncertainty about the relative importance of changes in speciation rates and changes in extinction rates will have profound implications for how hypothesis are constructed to explain the net diversification rate shifts discussed here. Further, a failure to sample extinct branches may 
mean that other aspects of the diversification history of Ipomoea have been entirely overlooked in this study.

\subsection{The sweet potato evolved in pre-human times}

We inferred time-calibrated phylogenies for Ipomoea in treePL using a point calibration for the root node that was equal to the MPE for the age of Ipomoea from Calibration Strategy 4. We suggest this calibration leads to significant underestimation of divergence times within Ipomoea, because the calibration strategy from which it is derived is likely to bias estimates to younger ages. Biasing our analysis in this way represents the most robust test of whether the storage root of I. batatas evolved in pre-human times. In these time-calibrated phylogenies, the divergence between $I$. batatas and its closest wild relative $I$. trifida was always in pre-human times. This was the case regardless of the smoothing value, or whether the phylogeny was inferred from nuclear or chloroplast data (Fig. S8a-d, Fig. S9ad). When we also inferred divergence times between I. batatas and its closest wild relatives in a Bayesian framework, the inferred divergence between I. batatas and I. trifida also significantly predated human times. This was the case regardless of whether a strict clock or a UCLN relaxed clock was used, or whether the phylogeny was inferred with nuclear or chloroplast data (Fig. 4, Fig. S8f, Fig. S9f). I. batatas is the only species amongst its close relatives that possesses a storage root, and together these results indicate that the storage root of $I$. batatas evolved in pre-human times.

We queried the conclusion that the divergence between I. batatas and I. trifida occurred in pre-human times by performing analyses with the chloroplast dataset where the divergence between I. batatas and I. trifida was constrained such that it occurred $15 \mathrm{Ka}$. In order to satisfy this calibration, we showed that the substitution rate for the chloroplast would be $2 * 10^{-2}$ substitutions $\mathrm{Myr}^{-1}$ (Fig. 5, Fig. S10). This is far higher than any previously recorded substitution or mutation rate for chloroplast DNA. Although there might be an expectation of elevated substitution rates for some genes in association with domestication, we would not expect such large rate differences to be observed across the entire plastome.

We also showed that the majority of diversity within I. batatas is likely to have evolved in pre-human times. In our time-calibrated phylogenies constructed from all sampled accessions of $I$. batatas and I. trifida, divergence events between different accessions significantly predated human times (Fig. 6, Fig. S11-S14). This was the case regardless of the method used. These different methods included using treePL with different smoothing values, Bayesian analyses with a strict clock and UCLN relaxed clock, and Bayesian analyses with 
three-taxon time-calibrated phylogenies that removed temporal assumptions associated with the birth-death tree prior.

\subsection{Assimilating uncertainty and making conclusions}

It is challenging to assimilate the uncertainty in the results presented here. By highlighting the assumptions that underpin different methods, the different possibilities of potential uncertainty can appear immense. In most studies performed in a Bayesian framework, the 95\% HPD is presented as an effective way of accounting for uncertainty. However, as demonstrated here, the 95\% HPD is entirely dependent on the assumptions that are made when performing an analysis. When the validity of different sets of assumptions is difficult to distinguish, as is often the case when inferring divergence times and diversification rates, no single 95\% HPD is therefore capable of reflecting the uncertainty inherent in these different assumptions. Thus, a more valuable approach for future studies, as followed here, may be to comprehensively account for the different types of assumptions that underlie inferences about the temporal dynamics of evolutionary diversification and openly discuss the implications of these. This may not provide simple answers to questions, but may provide a more robust foundation upon which future studies of macro-evolution can rest.

Despite the uncertainty we present here, we stress that when questions are carefully framed, and results carefully interpreted, novel and robust inferences about the temporal dynamics of evolutionary history can be made. In the analyses we present here, this includes the inference that the storage root of I. batatas evolved in pre-human times and was not a result of human domestication, and the inference that there is likely to be at least one significant shift in diversification rates within Ipomoea.

Regardless, in some cases it remains difficult to perform analyses from which robust inferences and confident conclusions about the temporal dynamics of evolutionary history can be made. Even when this is the case, for example with respect to the age of Ipomoea or major clades within Ipomoea, or the timing and frequency of diversification rate shifts, exploring the uncertainty in these inferences is invaluable. It can provide a basis upon which to develop new hypotheses that can be meaningfully tested with available data in the future, as well as providing a framework for prioritising the collection of new data. Further, it can stimulate theoretical and methodological studies that aim to characterise the extent to which inferences about macroevolutionary history can be made from analysing molecular phylogenies.

\section{FUNDING}


This work was supported by a NERC scholarship granted through the Environmental Research DTP programme to TC.

\section{REFERENCES}

Baldwin BG and Sanderson MJ. 1998. Age and rate of diversification of the Hawaiian silversword alliance (Compositae). Proc Natl Acad Sci USA. 95:9402-9406.

Britton T. 2005. Estimating Divergence Times in Phylogenetic Trees Without a Molecular Clock. Syst. Biol. 54:500-507.

Bromham L. 2006. Molecular dates for the Cambrian Explosion: is the light at the end of the tunnel an oncoming train? Palaeontol Electron. 9:2004-2006.

Cardillo M, Weston PH, Reynolds ZKM, Olde PM, Mast AR, Lemmon EM, Lemmon AR, Bromham L. 2017. The phylogeny and biogeography of Hakea (Proteaceae) reveals the role of biome shifts in a continental plant radiation. Evolution. 71:1928-1943.

Castresana J. 2000. Selection of conserved blocks from multiple alignments for their use in phylogenetic analysis. Mol Biol Evol. 17:540-552.

Crisp MD, Arroyo MTK, Cook LG, Gandolfo MA, Jordan GJ, McGlone MS, Weston PH, Westoby M, Wilf P, Linder HP. 2009. Phylogenetic biome conservatism on a global scale. Nature. 458:754756.

Dexter KG, Lavin M, Torke BM, Twyford AD, Kursar TA, Coley PD, Drake C, Hollands R, Pennington RT. 2017. Dispersal assembly of rain forest tree communities across the Amazon basin. Proc. Natl. Acad. Sci. USA. 114:2645-2650.

Donoghue MJ. 2008. A phylogenetic perspective on the distribution of plant diversity. Proc Natl Acad Sci USA. 105:11549-11555.

Donoghue MJ and Sanderson MJ. 2015. Confluence, synnovation, and depauperons in plant diversification. New Phytol. 207:260-274.

Doyle J. A., Biens P., Dorenkamp A., Jardiné S. 1977. Angiosperm pollen from the pre-Albian Cretaceous of Equatorial Africa. Bull. Cent. Rech. Explor. 1:451-473.

Drummond AJ, Ho SYW, Phillips MJ, Rambaut A. 2006. Relaxed Phylogenetics and Dating with Confidence. PLOS Biol. 4:e88.

Drummond AJ and Suchard MA. 2010. Bayesian random local clocks, or one rate to rule them all. BMC Biol. 8:114.

Fitch WM. 1976. Molecular evolutionary clocks. pp. 160-178 in Ayala FJ eds. Molecular Evolution. Sinauer: Sunderland.

Folk RA, Stubbs RL, Mort ME, Cellinese N, Allen J, Soltis PS, Soltis DE, Guralnick RP. 2019. Rates of niche and phenotype evolution lag behind diversification in a temperate radiation. Proc Natl Acad Sci USA. 116: 10874-10882

Gillespie J. H. 1991. The Causes of Molecular Evolution. Oxford: Oxford University Press.

Givnish TJ, MIllam KC, Mast AR, Paterson TB, Theim TJ, Hipp AL, Henss JM, Smith JF, Wood KR, Sytsma KJ. 2009. Origin, adaptive radiation and diversification of the Hawaiian lobeliads (Asterales: Campanulaceae). Proc. R. Soc. B. 276:407-416. 
Govindarajulu R, Hughes CE, Bailey CD. 2011. Phylogenetic and population genetic analyses of diploid Leucaena (Leguminosae; Mimosoideae) reveal cryptic species diversity and patterns of divergent allopatric speciation. Am. J. Bot. 98:2049-2063.

Hohna S, Heath TH, Boussau B, Landis MJ, Ronquist F, Huelsenbeck JP. 2014. Probabilistic Graphical Model Representation in Phylogenetics. Syst Biol. 63:753-771.

Hohna S, Landis MJ, Heath TA, Boussau B, Lartillot N, Moore BR, Huelsenbeck JP, Ronquist F. 2016. RevBayes: Bayesian Phylogenetic Inference Using Graphical Models and an Interactive ModelSpecification Language. Syst Biol. 65:726-736.

Huelsenbeck JP and Ronquist F. 2001. MRBAYES: Bayesian inference of phylogenetic trees. Bioinformatics. 17:754-755.

Hughes E. and Eastwood R. 2006. Island radiation on a continental scale: Exceptional rates of plant diversification after uplift of the Andes. Proc Natl Acad Sci USA. 103:10334-10339.

Hughes CE, Pennington RT, Antonelli A. 2013. Neotropical Plant Evolution: Assembling the Big Picture. Bot. J. Linn. Soc. 171:1-18.

Katoh K. 2002. MAFFT: a novel method for rapid multiple sequence alignment based on fast Fourier transform. Nucleic Acids Res. 30:3059-3066.

Katoh K and Standley DM. 2013. MAFFT Multiple Sequence Alignment Software Version 7: Improvements in Performance and Usability. Mol Biol Evol. 30:772-780.

Koenan EJM, de Vos JM, Aitchison GW, Simon MF, Schrire BD, de Sousa ER, de Queiroz LP, Hughes CE. 2013. Exploring the tempo of species diversification in legumes. S. Afr. J. Bot. 89:19-30.

Lavin M and Pennington RT. 2015. The contrasting nature of woody plant species in different neotropical forest biomes reflects differences in ecological stability. New Phytol. 2010:25-37.

Lavin M, Schrire BP, Lewis G, Pennington RT, Delgado-Salinas A, Thulin M, Hughes CE, Matos AB, Wojciechowski MF. 2004. Metacommunity process rather than continental tectonic history better explains geographically structured phylogenies in legumes. Philos. Trans. Royal Soc. 359:1509-1522.

Magallón S, Gómez-Acevedo S, Sánchez-Reyes LL, Hernández-Hernández T. 2015. A metacalibrated time-tree documents the early rise of flowering plant phylogenetic diversity. New Phytol. 207:437-453.

Magallón S, Hilu KW, Quandt D. 2013. Land plant evolutionary timeline: Gene effects are secondary to fossil constraints in relaxed clock estimation of age and substitution rates. Am J Bot. 100:556-573.

Mitchell TC, Williams BRM, Wood JRI, Harris DJ, Scotland RW, Carine MA. 2016. How the temperate world was colonised by bindweeds: biogeography of the Convolvuleae (Convolvulaceae). BMC Evol. Biol. 16:16.

Moore BR, Höhna S, May MR, Rannala B, Huelsenbeck JP. 2016. Critically evaluating the theory and performance of Bayesian analysis of macroevolutionary mixtures. Proc. Natl. Acad. Sci. USA. 113:9569-9574.

Morris JL, Puttick MN, Clark JW, Edwards D, Kenrick P, Pressel S, Wellman CH, Yang Z, Schneider H, Donoghue PCJ. 2018. The timescale of early land plant evolution. Proc Natl Acad Sci USA. 115:E2274-E2283.

Muñoz-Rodríguez P, Carruthers T, Wood JRI, Williams BRM, Weitemier K, Kronmiller B, Ellis D, Anglin NL, Longway L, Harris SA, Rausher MD, Kelly S, Liston A, Scotland RW. 2018. Reconciling Conflicting Phylogenies in the Origin of Sweet Potato and Dispersal to Polynesia. 28:1246-1256. 
Muñoz-Rodríguez P, Carruthers T, Wood JRI, Williams BRM, Weitemier K, Kronmiller B, Goodwin Z, Sumadijaya A, Anglin NL, Filer D, Harris D, Rausher MD, Kelly S, Liston A, Scotland RW. 2019. Assessing the biodiversity of morning glories at different scales using global natural history collections. Nat Plants, in press.

Nevado B, Atchison GW, Hughes CE, Filatov DA. 2016. Widespread adaptive evolution during repeated evolutionary radiations in New World lupins. Nat. Commun. 7:1-9.

Pennington RT, Lavin M, Oliveira-Filho A. 2009. Woody Plant Diversity, Evolution, and Ecology in the Tropics: Perspectives from Seasonally Dry Tropical Forests. 40:437-457. Annu. Rev. Ecol. Evol. Syst. 40:437-457.

Rabosky DL. 2014. Automatic detection of key innovations, rate shifts, and diversity-dependence on phylogenetic trees. PLOS ONE 9:e89543.

Rabosky DL, Chang J, Title OP, Cowmann PF, Sallan L, Friedman M, Kaschner K, Garilao C, Near TJ, Coll M, Alfaro ME. 2018. An inverse latitudinal gradient in speciation rate for marine fishes. Nature. 559:392-395.

Rabosky DL, Lovette IJ. 2008. Explosive Evolutionary Radiations: Decreasing Speciation or Increasing Extinction Through Time? Evolution. 62:1866-1875.

Rambaut A, Suchard MA, Xie D, Drummond AJ. 2014. Tracer v1.6 Available at: http://beast.bio.ed.ac.uk/Tracer

dos Reis M. and Yang Z. 2013. The unbearable uncertainty of Bayesian divergence time estimation. $J$. Syst. Evol. 51:30-43.

Ronquist F, Teslenko M, Van Der Mark P, Ayres DL, Darling A, Höhna S, Larget B, Liang L, Suchard MA, Huelsenbeck JP. 2012. MrBayes 3.2: Efficient Bayesian Phylogenetic Inference and Model Choice Across a Large Model Space. Syst Biol. 61:539-542.

Sanderson MJ. 1997. A Nonparametric Approach to Estimating Divergence Times in the Absence of Rate Constancy. Mol Biol Evol. 14:1218-1231.

Sanderson MJ and Doyle JA. 2001. Sources of error and confidence intervals in estimating the age of angiosperms from $r b c L$ and 18S rDNA data. Am. J. Bot. 88:1499-1516.

Särkinen T, Pennington RT, Lavin M, Simon MF, Hughes CE. 2012. Evolutionary islands in the Andes: persistence and isolation explain high endemism in Andean dry tropical forests. J Biogeogr. 39:884-900.

Särkinen T, Bohs L, Olmstead RG, Knapp S. 2013. A phylogenetic framework for evoluitonary study of the nightshades (Solanaceae): a dated 1000 tip tree. BMC Evol Biol. 13:214.

Simon MF, Grether R, de Queiroz LP, Skema C, Pennington RT, Hughes CE. 2009. Recent assembly of the Cerrado, a neotropical plant diversity hotspot, by in situ evolution of adaptations to fire. Proc. Natl. Acad. Sci. USA. 106:20359-20364.

Simoes AR, Culham A, Carine M. 2015. Resolving the unresolved tribe: a molecular phylogenetic framework for the Merremieae (Convolvulaceae). Bot. J. Linn. Soc. 179:374-387.

Smith SA and O'Meara BC. 2012. treePL: divergence time estimation using penalized likelihood for large phylogenies. Bioinformatics. 28:2689-2690.

Srivastava G, Mehrotra RC, Dilcher DL. 2018. Paleocene Ipomoea (Convolvulaceae) from India with implications for an East Gondwana origin of Convolvulaceae. Proc Natl Acad Sci USA. 115:60286033. 
Stefanović S, Austin DF, Olmstead RG. 2003. Classification of Convolvulaceae: A Phylogenetic Approach. Syst. Biol. 28:791-806.

Talavera G and Castresana J. 2007. Improvement of Phylogenies after Removing Divergent and Ambiguously Aligned Blocks from Protein Sequence Alignments. Syst Biol. 56:564-577.

Thorne JL, Kishino H, Painter IS. 1998. Estimating the rate of evolution of the rate of molecular evolution. Mol Biol Evol. 15:1647-1657.

Vaidya G, Lohman DJ, Meier R. 2011. SequenceMatrix: concatenation software for the fast assembly of multi-gene datasets with character set and codon information. Cladistics. 27:171-180.

Warnock RCM, Parham JF, Joyce WG, Tyler R, Donoghue PCJ. 2015. Calibration uncertainty in molecular dating analyses: there is no substitute for the prior evaluation of time priors. Proc R Sol B. 282:20141013.

Wilf P, Carvalho MR, Gandalfo MA, Ruben Cuneo N. 2017. Eocene lantern fruits from Gondwanan Patagonia and the early origins of Solanaceae. Science. 355:71-75.

Wilf P. and Escapa IH. 2015. Green Web or megabiased clock? Plant fossils from Gondwanan Patagonia speak on evolutionary radiations. New Phytol. 207:283-290.

Zuckerkandl E. and Pauling LB. 1962. Molecular disease, evolution, and genetic heterogeneity. pp. 189-225. in Kasha M. and Pullman B., eds. Horizons in biochemistry. Academic Press: New York.

Zuckerkandl E. and Pauling L. 1965. Evolutionary Divergence and Convergence. pp. 97-166 in Bryson V. and Vogel HJ., eds. Evolving genes and proteins. Academic Press: New York.

Zhu T., dos Reis M., Yang Z. 2015. Characterization of the Uncertainty of Divergence Time Estimation under Relaxed Molecular Clock Models Using Multiple Loci. Syst. Biol. 64:267-280.

\section{FIGURE CAPTIONS}

Figure 1. A summary of analyses performed in this study. The dark grey boxes in the centre of the figure illustrate the progression from a sequence alignment, to a phylogeny, to a timecalibrated phylogeny, to inferring diversification parameters. The light grey boxes summarise the assumptions and issues associated with each step, and the way in which they were accounted for in this study.

Figure 2. Time-calibrated phylogenies for Convolvulaceae and Solanaceae inferred with different calibration strategies and a UCLN relaxed clock. Branch colours refer to the inferred substitution rate (MPE). Black circles refer to calibrated nodes. The grey "1" refers to the Solanaceae stem node (divergence between Solanaceae and Convolvulaceae), the grey " 2 " refers to the Convolvulaceae crown node, the grey " 3 " refers to the Solanaceae crown node, the grey "4" refers to the Ipomoea crown node. Grey bars refer to 95\% HPD intervals for the ages of these nodes. The time axis includes the geological period: $\mathrm{K}=$ Cretaceous, $\mathrm{P}=$ Paleogene, $\mathrm{Ng}=$ Neogene, $\mathrm{Q}=$ Quaternary. a) Calibration Strategy 1 - the Solanaceae stem node has a maximum age constraint of $130 \mathrm{Myr}$, and the Solanoideae stem node has a minimum age constraint of 52Myr. b) Calibration Strategy 2 - the Solanaceae stem node is calibrated with a lognormal distribution with offset $=52, m=10.4, v=10.4$. The Solanoideae stem node has a minimum age constraint of 52Myr. c) Calibration Strategy 3 - the Solanaceae stem node is calibrated with a lognormal distribution with offset $=52, m=5.2, v$ $=2.1$. The Solanoideae stem node has a minimum age constraint of 52Myr. d) The Solanaceae 
stem node is calibrated with a secondary calibration from Magallon et al. (2015). This is implemented as a normal distribution with $\mu=67.34, \sigma=9.980$.

Figure 3. Time-calibrated phylogenies for Ipomoea inferred in treePL with the optimum smoothing value, and images of species from different clades. A point calibration of 30.3 Myr is used at the root node in both a) and b). This is the MPE for the age of Ipomoea from Calibration Strategy 1. Where rates differ between branches, branch colours refer to the inferred substitution rate. Labelled nodes refer to nodes defining major clades. A $-E$ refer to clades A - E from Muñoz-Rodríguez et al. (2019), IA refers to Astripmoea and close relatives, NW and OW refer to the New World and Old World clades (see Muñoz-Rodríguez et al. 2019), par refers to the Parana Clade, $c s$ refers to the Coriaceous Sepal Clade, and bat refers to the Batatas group. Labelled arrows refer to the positions of shifts in diversification parameters inferred in BAMM. The number next to each arrow refers to the posterior probability of the shift occurring at that position in the phylogeny. Only shifts with a posterior probability of $>0.05$ are indicated. The time axis includes the geological epoch: $\mathrm{Ol}$ = Oligocene, $\mathrm{M}=$ Miocene, $\mathrm{P}=$ Pliocene, $\mathrm{Ps}=$ Pleistocene, and $\mathrm{H}=$ Holocene. a) shows a nuclear time-calibrated phylogeny, and b) shows a chloroplast time-calibrated phylogeny. $\mathbf{c}$ ) I. cordatotriloba, a trailing and twining species in the Batatas group. d) I. nil, a twining species in clade B. e) I. cairica, a trailing and twining species in clade E. f) I. bonariensis, a trailing and twining species in the Coriaceous Sepal Clade. g. I. paraguariensis, an erect species in the Parana clade. h) I. paludosa, an erect species in the Parana clade. i. $I$. psammophila, a trailing species in the Parana clade. j) I. megapotamica, a trailing and climbing species in the Parana clade.

Figure 4. Time-calibrated phylogenies for the Batatas group inferred in RevBayes with a UCLN relaxed clock. Branch colours refer to the inferred substitution rate. The grey bar refers to the 95\% HPD for the divergence between I. batatas and I. trifida. a) shows the nuclear time-calibrated phylogeny, b) shows the chloroplast time-calibrated phylogeny.

Figure 5. Alternative chloroplast time-calibrated phylogenies for the Batatas group inferred in RevBayes. Where rates differ between branches, branch colours refer to the inferred substitution rate. In a) the divergence between I. batatas and I. trifida is calibrated to $15 \mathrm{Kyr}$ and the root node has a point calibration of $6.18 \mathrm{Myr}$. This is the same root calibration as was used in all the previous time-calibrated phylogenies for the Batatas group inferred in RevBayes. A UCLN relaxed clock is used to infer substitution rates. In b) the divergence between I. batatas and I. trifida is constrained to have occurred $15 \mathrm{Ka}$ and there is no calibration at the root node. A strict clock is used to infer substitution rates.

Figure 6. Time-calibrated phylogenies for I. batatas and I. trifida inferred in treePL with the optimum smoothing value. All sampled accessions of the two species are included. Branch colours refer to the inferred substitution rate. a) shows the nuclear time-calibrated phylogeny, b) shows the chloroplast time-calibrated phylogeny. 
1 The temporal dynamics of evolutionary diversification in Ipomoea

2 Tom Carruthers ${ }^{1}$, Pablo Muñoz-Rodríguez ${ }^{1}$, John R. I. Wood ${ }^{1}$, and Robert W.

3 Scotland $^{1 *}$

4 Affiliations:

$5{ }^{1}$ Department of Plant Sciences, University of Oxford, South Parks Road, Oxford, OX1 3RB,

6 United Kingdom

$7 \quad$ * Correspondence to be sent to: Department of Plant Sciences, University of Oxford, South

8 Parks Road, Oxford, OX1 3RB, United Kingdom; E-mail: robert.scotland@plants.ox.ac.uk

9

10

11

12

13

14

15

16

17

18

19

20

21

22

23

24

25

26

27

28 


\section{Abstract}

30 Molecular phylogenies are used as a basis for making inferences about macroevolutionary history. However, a robust phylogeny does not contain the information that is necessary to make many of these inferences. Complex methodologies that incorporate important assumptions about the nature of evolutionary history are therefore required. Here, we explore the implications of these assumptions for making inferences about the macroevolutionary history of Ipomoea - a large pantropical genus of flowering plants that contains the sweet potato (Ipomoea batatas), a crop of global economic importance. We focus on assumptions that underlie inferences of divergence times, and diversification parameters (speciation rates, extinction rates, and net diversification rates). These are among the most fundamental variables in macroevolutionary research. We use a series of novel approaches to explore the implications of these assumptions for inferring the age of Ipomoea, the ages of major clades within Ipomoea, whether there are significant differences in diversification parameters among clades within Ipomoea, and whether the storage root of I. batatas evolved in pre-human times. We show that inferring an age estimate for Ipomoea and major clades within Ipomoea is highly problematic. Inferred divergence times are sensitive to uncertain fossil calibrations and differing assumptions about among-branch-substitution-rate-variation. Despite this uncertainty, we are able to make robust inferences about patterns of variation in diversification parameters within Ipomoea, and that the storage root of I. batatas evolved in pre-human times. Taken together, this study presents novel and generalizable insights into the implications of methodological assumptions for making inferences about macroevolutionary history. Further, by presenting novel findings relating to the temporal dynamics of evolution in Ipomoea, as well as more specifically to I. batatas, this study makes a valuable contribution to our understanding of tropical plant evolution, and the evolutionary context in which economically important crops evolve.

Key words: phylogenetics, evolution, divergence times, diversification, Ipomoea, Sweet Potato 


\section{INTRODUCTION}

Molecular phylogenies are of central importance to macroevolutionary research. Our understanding of evolutionary radiations, and more generally, the factors that affect when, how, and why diversity accumulates in different clades, is underpinned by the interpretation of molecular phylogenies in an evolutionary context (Baldwin and Sanderson 1998; Hughes and Eastwood 2006; Givnish et al. 2009; Rabosky et al. 2008; Koenan et al. 2013; Donoghue and Sanderson 2015; Nevado et al. 2016; Rabosky et al. 2018). So too is our understanding of when and how the earth's major biomes were assembled (Pennington et al. 2009; Simon et al. 2009; Govindarajulu et al. 2011; Särkinen et al. 2012; Hughes et al. 2013; Pennington and Lavin 2015; Dexter et al. 2017) and the historical processes that have shaped distribution patterns such as long-distance-dispersal and geological vicariance (Lavin et al. 2004; Donoghue 2008; Crisp et al. 2011; Muñoz-Rodríguez et al. 2018). Because of their importance to macroevolutionary research, the rapid increase in availability of robust molecular phylogenies for many groups has enabled macroevolutionary questions to be investigated in greater detail and a greater variety of different contexts.

There are fundamental differences between a robust molecular phylogeny and the evolutionary process that it is used as a basis for making inferences about. These differences mean that even if a robust phylogeny is known for a group, parameters of interest in macroevolutionary research such as divergence times, net diversification rates, speciation rates, and extinction rates can be inferred with significant error (Sanderson and Doyle 2001; Britton 2005; Rabosky 2010; Moore et al. 2016; Wilf and Escapa 2015; Carruthers et al. 2019). It is crucial to account for this error, especially when using inferences as a basis for constructing narratives or explanations about when, how and why a group evolved. If error is not adequately accounted for, complex narratives may be constructed that are not supported by available evidence (Wilf and Escapa 2015).

Here, we use the recently developed phylogenetic framework for Ipomoea (MuñozRodríguez et al. 2019) to investigate the temporal dynamics of its evolutionary diversification. Ipomoea is a pantropical genus of over 800 species that exhibit a range of growth forms including climbers, trailers, erect shrubs and trees. They also occur in a range of habitats including cerrado, dry forest, and disturbed wasteland (Wood et al. 2015). Further, the genus contains the sweet potato (I. batatas), a crop of global economic importance (Muñoz-Rodríguez et al. 2018). Investigating the temporal dynamics of evolutionary diversification in Ipomoea can therefore provide critical insights into the nature of 
evolutionary diversification in large tropical groups, and when, how and why important crops evolve from their wild relatives.

In this study, we specifically focus on the inference of divergence times, net diversification rates, speciation rates, and extinction rates. Net diversification rates, speciation rates, and extinction rates are subsequently referred to collectively as diversification parameters. We focus on divergence times and diversification parameters because these two variables are the fundamental basis upon which other aspects of macroevolutionary research depend. When inferring these two variables, we explicitly consider different sources of uncertainty. With respect to divergence times, one of the primary sources of uncertainty are fossil calibrations (Sanderson and Doyle 2001; dos Reis and Yang 2013; Magallón et al 2013; Warnock et al. 2015; Morris et al. 2018). These are likely to be a particularly important source of uncertainty for Ipomoea, and many other angiosperm genera, because their fossil records are extremely fragmentary (Särkinen et al. 2013; Mitchel et al. 2016; Cardillo et al. 2017; Wilf et al. 2017; Folk et al 2019). It is therefore difficult to make robust interpretations of the temporal signal in the fossil record, and to use it as a basis to calibrate phylogenies. For divergence times, we also consider the effect of different assumptions about substitution rates and the magnitude of among-branchsubstitution-rate-variation (Zuckerkandl and Pauling 1962, 1965; Fitch 1976; Gillespie 1991; Sanderson 1997; Thorne et al. 1998; Britton 2005; Bromham 2006; Drummond et al. 2006; Zhu et al. 2015; Carruthers et al. 2019), as well as the effect of assuming constant diversification parameters when performing divergence time analyses in a Bayesian framework. For the inference of diversification parameters, we explore the effect of divergence time estimation error on inferred diversification parameters. We also discuss the effects of limited taxon sampling, and the effects of extinct branches that are un-sampled in a molecular phylogeny.

When exploring these sources of uncertainty in the context of Ipomoea, we investigate several questions that relate to the timing and tempo of evolutionary diversification in the entire genus. These include the age of Ipomoea, the ages of major clades within Ipomoea, diversification parameters within Ipomoea, and the extent to which diversification parameters vary among clades within Ipomoea. Given the recently developed phylogenetic framework for Ipomoea resolves the relationships between I. batatas and its closest wild relatives (Muñoz-Rodríguez et al. 2018), we also investigate questions relating to the origin of I. batatas. This includes when I. batatas and its storage root evolved, and 
specifically, whether it evolved in pre-human times, or whether it evolved more recently as a likely result of human domestication.

\section{MATERIALS AND METHODS}

\subsection{Estimating the age of Ipomoea}

To estimate the age of Ipomoea, we inferred a time-calibrated phylogeny for Convolvulaceae, which contains Ipomoea, and its sister family Solanaceae, which has a richer fossil record (Särkinen et al. 2013; Wilf et al 2017). This time-calibrated phylogeny was based on a molecular dataset of 4 chloroplast genes $(a t p B, m a t K, n d h F, r b c L)$, the chloroplast intergenic spacer $\operatorname{trnL-trnF}$, and the nuclear ITS marker. Apart from sampled species of Ipomoea - for which we used sequences from Muñoz-Rodríguez et al. 2019, sampled sequences were downloaded from GenBank (Table S1). A total of 56 taxa were sampled to represent the phylogenetic breadth of the two families. We purposefully sampled a low proportion of taxa, and an equal number of taxa from each sister family. The rationale for this was to reduce the effect of violating the assumption of the constant rate birth-death branching process that the diversification parameters are constant - an underlying assumption of most Bayesian methods of divergence time estimation. This is because sampling an equal number of taxa from each sister clade will likely cause diversification parameters to be constant amongst sampled taxa at broad sales across the phylogeny, whilst sampling a small number of taxa will mean that any violation of the assumption of constant diversification parameters amongst sampled taxa will have a statistically weaker effect on divergence time estimates.

We aligned sequences in MAFFT v7.271 (Katoh 2002; Katoh and Standley 2013) using the L-INS-I setting and a gap opening penalty of 1.53. Ambiguously aligned regions were removed using default settings in Gblocks (Castresana 2000; Talavera and Castresana 2007), and Sequence Matrix v1.8 (Vaidya et al. 2011) was used to concatenate aligned sequences. The final concatenated matrix had 5,166 sites for the 56 taxa. We used MrBayes v3.2.6 (Huelesenbeck and Ronquist 2001; Ronquist et al. 2012) to infer the tree topology for subsequent divergence time analyses. A GTR + G + I model was used. This analysis was run for 2,000,000 generations and the final split frequency between runs was $<0.01$. The topology we recovered is consistent with previous phylogenetic studies of these two families (Stefanović et al. 2003; Särkinen et al. 2013; Simoes et al. 2015; Mitchell et al. 2016). The validity of different temporal calibrations for Convolvulaceae and Solanaceae is unclear. We therefore experimented with different calibration strategies. However, in no case did we use any fossil calibrations within Ipomoea because there are no fossils that can 
reliably be assigned to the genus. This includes the recently published leaf fossils of Ipomoea meghalayensis (Srivastava et al. 2018). This is because the short thick petioles of these fossils are not typical of Ipomoea; their well-preserved gland dots, leaf venation, and cordate leaves are characteristic of several other genera; and from the fossils alone it cannot be determined that the plant has alternate leaves, as would be expected for Ipomoea (Hawthorne pers. comm.)

In Calibration Strategy 1, 2 and 3, the recently discovered fossil, Physalis infinemundi (Wilf et al. 2017) was used as a basis for implementing fossil calibrations. This fossil - dated at around 52 million years (Myr) - is far older than fossil calibrations previously used in Convolvulaceae and Solanaceae, and is also considerably older than previous age estimates for either of these two families (Särkinen et al. 2013; Magallón et al. 2015; Mitchell et al. 2016). Because of this, it places previous assumptions about the timing of evolution within these clades, and the relationship between fossil ages and clade ages, into considerable doubt.

In Calibration Strategy 1, both the Solanoideae stem node and Solanaceae stem node (root node) are calibrated with a uniform distribution $(\mathrm{min}=52$ million years ago $(\mathrm{Ma}), \max$ $=130 \mathrm{Ma}$ ). The minimum corresponds to the age of Physalis infinemundi, and the maximum corresponds to the time that eudicot pollen suddenly appears in the fossil record (Doyle et al 1977). This calibration strategy assumes the Solanoideae stem node is older than $52 \mathrm{Myr}$, and the Solanaceae stem node is younger than $130 \mathrm{Myr}$. Apart from this, these calibrations do not make explicit assumptions about where the most probable clade ages lie between the minimum and maximum constraints.

In Calibration Strategy 2 and 3, we accounted for the fact that $130 \mathrm{Myr}$ is most probably an overestimate of the Solanaceae stem node age - at least in the context of current understanding of the temporal dynamics of plant diversification (Särkinen et al. 2013; Magallón et al. 2015; Mitchell et al. 2016). We therefore calibrated the Solanaceae stem node with a lognormal calibration density parametrised as either: offset $=52, m=10.4, v=10.4$ (Calibration Strategy 2); or offset $=52, m=5.2, v=2.1$ (Calibration Strategy 3). In Calibration Strategies 2 and 3, the minimum constraint of $52 \mathrm{Ma}$ is retained at the Solanoideae stem node. The effect of Calibration Strategies 2 and 3 is therefore to shift the calibration density at the Solanaceae stem node to ages that are significantly younger than 130 Myr, with Calibration Strategy 3 shifting the distribution to younger ages than Calibration Strategy 2. 

most realistic, given that there is no evidence upon which to derive assumptions about how much younger than $130 \mathrm{Myr}$ the Solanaceae stem node is. Comparing these three Calibration Strategies therefore provides a basis to evaluate the implications of different assumptions about the likely age of the Solanaceae stem node.

In Calibration Strategy 4, we calibrated the Solanaceae stem node with a normal distribution $(\mu=67.34, \sigma=9.980)$. This is a secondary calibration from Magallón et al. (2015) in which 132 fossil calibrations were used throughout Spermatophyta. This calibration strategy is therefore not entirely dependent on the interpretation of fossil calibrations within Convolvulaceae and Solanaceae. Characteristics of the analysis from which Calibration Strategy 4 is derived (Magallón et al. 2015) are likely to bias estimates to younger ages. For example, this study implements an extremely young maximum constraint on the angiosperm crown age, and employs calibration densities throughout the phylogeny which assume that fossil ages are very close to clade ages. Therefore, age estimates derived from Calibration Strategy 4 were primarily used with respect to the evolution of the sweet potato. This is because biasing estimates to younger ages provided the most robust test of whether the storage root of the sweet potato evolved in pre-human times - one of the main hypotheses that we set out to examine.

We used RevBayes v1.0.4 (Hohna et al. 2014; Hohna, et al. 2016) to infer timecalibrated phylogenies according to the four calibration strategies. In all analyses, a GTR $+G$ + I model was used with a birth-death branching process as the tree prior. The topology was fixed to that inferred in MrBayes, and starting trees consistent with the 4 different calibration strategies were generated in treePL (Smith and O’Meara 2012). In one set of analyses, a strict clock was used where the substitution rate was assumed to be the same for every branch. In a second set of analyses, an uncorrelated lognormal (UCLN) relaxed clock (Drummond et al. 2006) was used. For each analysis, two independent runs were performed. Sufficient mixing and convergence was assessed in Tracer v1.6.0 (Rambaut et al. 2014). A 25\% burn-in was used prior to calculating maximum a posteriori (MAP) trees, mean posterior estimates (MPEs), and 95\% highest posterior density intervals (HPDs).

\subsection{Inferring the ages of major clades within Ipomoea}

We used the mean posterior estimate (MPE) for the age of Ipomoea from Calibration Strategy 1 as a point calibration for the root node of more densely sampled phylogenies for the genus. We used this MPE because the calibration strategy from which it is derived does not make any explicit assumptions about probable clade ages in relation to minimum or 
maximum constraints. It can therefore act as a basis from which to discuss the implications of calibration strategies that make explicit assumptions about probable clade ages in relation to minimum or maximum constraints.

Because we do not use further fossil calibrations within Ipomoea, we can easily interpret the implications of root node calibrations for Ipomoea derived from different calibration strategies - the relative ages of nodes within the time-calibrated phylogeny will not change, only the absolute timescale over which the time-calibrated phylogeny occurs. This also explains why we use a point calibration for the root node, rather than a calibration density. We can easily compare the effects of root node calibrations that derive from different calibration strategies. By contrast, it would be difficult to construct a single calibration density that adequately accounts for uncertainty in root node estimates that derive from each of the calibration strategies outlined above.

For divergence time analyses within Ipomoea, we were interested in estimating the ages of major clades from Muñoz-Rodríguez et al. (2019); and also the Parana Clade - a diverse clade centred in central South America; the Coriaceous Sepal Clade - a diverse clade centred in the Caribbean; and the Batatas group - the clade of I. batatas and its closest wild relatives. We were also interested in constructing a temporal framework for subsequent analyses of diversification parameters.

For these divergence time analyses, we used the molecular datasets and phylogenies from Muñoz-Rodríguez et al. 2019. This includes a nuclear dataset of 434 single copy genes for 211 species of Ipomoea, and a plastome dataset of 206 species of Ipomoea. Within these more densely-sampled datasets, there may be significant differences in diversification parameters among clades. This violates a key assumption of Bayesian methods of divergence time estimation that use a constant rate birth-death branching process as the tree prior. We therefore used treePL to infer divergence times - a penalised likelihood method that does not use a constant rate birth-death branching process (Smith and O'Meara 2012). In treePL, we performed analyses with the nuclear and chloroplast phylogenies from Muñoz-Rodríguez et al. (2019) as input trees. Following extensive cross-validation, we used a smoothing value of 0.01 for the nuclear phylogeny, and 10000 for the chloroplast phylogeny (lower smoothing values assume more among-branch-substitution-rate-variation than higher smoothing values). For both phylogenies, we also inferred time-calibrated phylogenies with alternative smoothing values (1, 100 and 10000 for nuclear, and 0.01, 1 and 100 for chloroplast). By experimenting with different smoothing values, we were able to determine the sensitivity of 
divergence time estimates to different assumptions about the magnitude of among-branchsubstitution-rate-variation.

Inferred divergence times within Ipomoea may be sensitive to the unique characteristics of different methods - especially given there are no calibrations within Ipomoea with which to constrain age estimates. We therefore also used RevBayes to estimate divergence times within Ipomoea in two sets of analyses.

In one set of analyses, we constructed skeletal time-calibrated phylogenies for Ipomoea with the taxon-sampling designed such that the ages of clades of interest (set out above) were estimated. For the nuclear data, we sampled all genes that were sampled in every taxon in the skeletal phylogeny (36 taxa) and for which the aligned sequence length was at least 4,000 sites. For the chloroplast data, we sampled the entire plastomes for 37 taxa.

Divergence time estimates in the skeletal time-calibrated phylogenies may be biased by the tree prior which assumes constant diversification parameters when inferring divergence times. In the second set of analyses performed in RevBayes, a series of three taxon time-calibrated phylogenies was therefore inferred, the divergence times within which would not be affected by the assumptions of the tree prior. The taxa sampled within these three-taxon phylogenies was such that the root node of Ipomoea was always sampled, along with one further node from within Ipomoea that uniquely corresponded to a node within the skeletal phylogenies. Thus, for each node in the skeletal phylogenies, we generated two age estimates - one derived from the skeletal phylogeny itself, and one from the three-taxon phylogeny that sampled that node. For three-taxon phylogenies constructed from nuclear data, we sampled all genes sampled for all three species in each three-taxon phylogeny. For the chloroplast data, we sampled the entire plastomes for the three species sampled in each three-taxon phylogeny.

In both the skeletal and three-taxon time-calibrated phylogenies, the MPE for the age of Ipomoea from Calibration Strategy 1 was used as a point calibration for the root node. In both sets of analyses, we also used the same alignments as Muñoz-Rodríguez et al. (2019). For the skeletal phylogenies, the total aligned sequence length was 39,495 sites for the nuclear data, and 103,921 sites for the chloroplast data. We used a GTR + G + I model of sequence evolution, and both a strict clock and a UCLN relaxed clock. A birth-death process was used as the tree prior for the skeletal phylogenies. A Yule process was used as the tree prior for the three-taxon phylogenies. The Yule process was parameterised such that the prior probability for the age of the internal node - the single unknown divergence time - was uniformly distributed between the root age and the present. Divergence time estimates from 
the three-taxon phylogenies are not therefore biased by the birth-death branching process. In all analyses, the topologies were fixed such that they were congruent with those inferred by Muñoz-Rodríguez et al. (2019). Two independent runs were performed. Sufficient mixing and convergence was assessed in Tracer v1.6.0. A 25\% burn-in was used prior to calculating MAP trees, MPEs and 95\% HPDs.

\subsection{Inferring diversification parameters within Ipomoea}

We inferred diversification parameters in BAMM (Rabosky 2014). BAMM is a Bayesian method that determines the probability of different patterns of discrete shifts in diversification parameters occurring in a time-calibrated phylogeny. Current knowledge of diversification parameters within Ipomoea is very limited, whilst understanding of habit preferences, geographical distributions, and relationships amongst species is in some cases far from complete. Analyses of diversification parameters within Ipomoea were therefore exploratory. Nonetheless, one particular hypothesis we were interested in testing was whether the Parana Clade, which is very diverse and possesses species with overlapping morphologies and narrow distribution ranges, has significantly increased speciation rates.

We used the 8 time-calibrated phylogenies inferred in treePL (smoothing values of $0.01,1,100,10000$ with nuclear and chloroplast phylogenies) as input trees. We could therefore evaluate how different assumptions about among-branch-substitution-rate-variation (and by extension differences in divergence time estimates), as well as topological differences between the nuclear and chloroplast phylogenies, affect inferred diversification parameters. Following consultation with John Wood, who has carried out the majority of the taxonomic work in this project, we specified approximate sampling proportions for different clades. We used the set_priors.R script - downloaded with BAMM and requiring the $\mathrm{R}$ package BAMMtools - to select the appropriate priors. Four independent runs were performed for each analysis. For the chloroplast time-calibrated phylogeny inferred with a smoothing value of 0.01 , the analysis was run for 4,000,000 generations. For the chloroplast time-calibrated phylogeny inferred with a smoothing value of 1 , and the nuclear timecalibrated phylogeny inferred with a smoothing value of 0.01 , the analyses were run for $2,000,000$ generations. In all other cases, the analyses were run for 1,000,000 generations. A $10 \%$ burnin was used. Convergence was assessed using a custom R script that required the package BAMMtools. We compared Bayes factors between different models to identify the number of shifts in diversification parameters that occurred within our time-calibrated phylogenies. We then extracted the credible shift set - which represents the set of trees with 
different diversification parameter shift patterns that account for $95 \%$ of the posterior probability.

\subsection{When did the sweet potato evolve?}

To answer questions relating to the age of I. batatas, we used the MPE for the age of Ipomoea from Calibration Strategy 4 as a point calibration for the root node of timecalibrated phylogenies for Ipomoea. Calibration Strategy 4 does not account for the recently discovered and surprisingly old Physalis infinemundi. Further, several characteristics of the analysis (Magallón et al. 2015) from which Calibration Strategy 4 is derived are likely to mean that age estimates from this analysis are biased to younger ages. Age estimates derived from this calibration strategy are therefore likely to be considerably younger than those derived from Calibration Strategies 1 and 2 (and potentially 3). With respect to I. batatas, we are especially interested in determining whether its storage root evolved in pre-human times. Biasing our analysis to younger ages - by using Calibration Strategy 4 - is one of the most robust ways to do this, especially if, despite biasing analyses to younger ages, the recovered divergence times are pre-human.

We used this point calibration to calibrate the nuclear and chloroplast phylogenies for Ipomoea from Muñoz-Rodríguez et al. (2019). The time-calibrated phylogenies were inferred in treePL. The same optimum and alternative smoothing values were used as outlined previously.

The taxon sampling within these time-calibrated phylogenies is sufficient to estimate a divergence time between I. batatas and its closest wild relative I. trifida. Given I. trifida does not possess a storage root, the estimated divergence time between these two species can provide a basis for understanding when the storage root of I. batatas is likely to have evolved.

To scrutinise these divergence time estimates, we inferred additional time-calibrated phylogenies for the Batatas group in RevBayes. We could therefore explore the sensitivity of divergence time estimates in different methodological frameworks.

In an initial set of analyses in RevBayes, we used a point calibration for the root node (of the Batatas group) that was equal to the age estimate for the equivalent node in the nuclear time-calibrated phylogeny (for the entire genus) inferred with the optimum smoothing value in treePL. We inferred these phylogenies in RevBayes with both nuclear and chloroplast data from Muñoz-Rodríguez et al. (2018). For the nuclear data, we sampled all genes that were sampled in every species in the Batatas group. For the chloroplast data, we sampled the entire plastomes of every species in the Batatas group, except I. lactifera. The 
plastome of I. lactifera is highly reduced, and several problems resulted from its inclusion in preliminary analyses. We used the same alignments as Muñoz-Rodríguez et al. (2018). The total aligned sequence length was 375,628 sites for the nuclear data, and 160,435 sites for the chloroplast data. For both datasets, we used a GTR + G + I model of sequence evolution, and both a strict clock and a UCLN relaxed clock. We used a constant rate birth-death branching process as the tree prior. The topologies were fixed such that they were consistent with those inferred by Muñoz-Rodríguez et al. (2018). For each time-calibrated phylogeny, two independent runs were performed. Sufficient mixing and convergence was assessed in Tracer v1.6.0. A 25\% burn-in was used prior to calculating MAP trees, MPEs, and 95\% HPDs.

In a further set of analyses in RevBayes, the divergence between I. batatas and $I$. trifida was constrained such that it occurred $15 \mathrm{Ka}$. This is consistent with a timeframe that could allow for the storage root of $I$. batatas to be a product of domestication, given that the earliest evidence for crop domestication is a little over $10 \mathrm{Ka}$ (Feldman 2001; Hillman et al. 2001; Zohary et al. 2006; Kingsbury 2009). By using this constraint, we could infer the substitution rates required for the storage root of I. batatas to have evolved in human times as a result of domestication.

We performed two variants of this analysis. In the first, the root node of the Batatas group had the same point calibration as other analyses in this section. We therefore assume that the temporal framework inferred for the entire genus is broadly accurate. In the second, the root node did not have a point calibration. We therefore make no assumptions about the accuracy of the temporal framework inferred for the entire genus. This second variant is instead entirely independent of this temporal framework.

Where the root node had a point calibration, we expect our analysis may infer major differences in substitution rates between branches. As such we performed this analysis with both a UCLN relaxed clock - where substitution rates for each branch are drawn from a single continuous distribution, and a random local clock - where discrete (and potentially very large) changes in substitution rates between different branches are explicitly modelled (Drummond and Suchard 2011). Where the root node was not fixed, we used a strict clock. This additional set of analyses was performed with only the chloroplast data. As before, we used a GTR + G + I model of sequence evolution and a birth-death branching process as the tree prior. The topologies were fixed such that they were consistent with those inferred by Muñoz-Rodríguez et al. (2018). For each time-calibrated phylogeny, two independent runs were performed. Sufficient mixing and convergence was assessed in Tracer v1.6.0. A 25\% burn-in was used prior to calculating MAP trees, MPEs, 95\% HPDs. 

possess storage roots (Munoz et al. 2019). To provide further context for understanding the temporal dynamics of storage root evolution within Ipomoea, and determine whether there is

400

401

402

403

404

405

406

407

408

409

410

411

412

413

414

415

416

417

418

419

420

421

422

423

424

425

426

427

428

429 the capacity for them to evolve in pre-human times, we estimated when Ipomoea taxa with storage roots are likely to have diverged from relatives that do not possess storage roots. This provides a basis for understanding when storage roots are likely to have evolved throughout Ipomoea. These estimates were based on the nuclear time-calibrated phylogenies inferred in treePL with different smoothing values.

As well as estimating the divergence time between I. batatas and I. trifida, we also estimated the age of diversity within I. batatas. To do this, we inferred time-calibrated phylogenies with multiple accessions of I. batatas (71 accessions) and I. trifida (22 accessions). We inferred these time-calibrated phylogenies in treePL and RevBayes.

For analyses in treePL, we used nuclear and chloroplast phylogenies that contain multiple accessions of I. batatas and I. trifida (from Muñoz-Rodríguez et al. 2018) as input trees. We used a point calibration for the root node that was equal to the age estimate for the equivalent node in the nuclear time-calibrated phylogeny for the entire genus inferred with the optimum smoothing value in treePL. Following extensive cross-validation, we used a smoothing value of 0.01 for the nuclear and chloroplast phylogenies. We also inferred timecalibrated phylogenies with alternative smoothing values $(1,100,10000)$ to determine the sensitivity of divergence time estimates to different assumptions about the magnitude of among-branch-substitution-rate-variation.

In RevBayes, we performed two sets of analyses. First, we inferred time-calibrated phylogenies for all sampled accessions with either the nuclear or chloroplast data. For the nuclear data, we sampled the 40 genes that were sampled in every accession with the longest aligned sequence length. For the chloroplast data, we sampled entire plastomes. Second, we inferred a series of three-taxon time calibrated phylogenies with either the nuclear or chloroplast data. The motivation for this was the same as set out for the three-taxon timecalibrated phylogenies inferred across the whole of Ipomoea. The taxon sampling was such that in each three-taxon phylogeny, the root node of the phylogeny containing all sampled accessions was always sampled, along with one further node. For the nuclear data, we sampled all genes that were sampled for all three species in each three-taxon phylogeny. For the chloroplast data, we sampled the entire plastome for the three species sampled in each three-taxon phylogeny. We used the same alignments as Muñoz-Rodríguez et al. (2018). 
In all these analyses, a GTR + G + I model of sequence evolution was used. We used either a strict clock or a UCLN relaxed clock. We used a point calibration for the root node that was equal to the age estimate for the equivalent node in the nuclear time-calibrated phylogeny for the entire genus inferred with the optimum smoothing value in treePL. For the time-calibrated phylogenies containing all sampled accessions, a birth-death branching process was used as the tree prior. For the three-taxon time-calibrated phylogenies, a Yule process was used as the tree prior. This was parameterised such that the prior probability for the age of the internal node - the single unknown divergence time - was uniformly distributed between the root age and the present. In all analyses, the topologies were fixed such that they were consistent with those inferred by Muñoz-Rodríguez et al. 2018. For each time-calibrated phylogeny, two independent runs were performed. Sufficient mixing and convergence was assessed in Tracer v1.6.0. A 25\% burn-in was used prior to calculating MAP trees, MPEs and 95\% HPDs.

\subsection{A comprehensive analysis of uncertainty in our understanding of the temporal} dynamics of evolutionary diversification in Ipomoea

The purpose of the large array of analyses presented here is to comprehensively evaluate and account for the different sources of uncertainty when inferring divergence times, and subsequently, diversification parameters. These different sources of uncertainty are highly interlinked and complex. In Figure 1, we summarise these different sources of uncertainty, and the ways in which we have accounted for them in this study.

\section{RESULTS}

\subsection{Time-calibrated phylogenies for Solanaceae and Convolvulaceae, and an age estimate for Ipomoea}

Here, we summarise results when a UCLN relaxed clock is used with different calibration strategies for Convolvulaceae and Solanaceae, followed by results when using a strict clock.

Calibration Strategy 1 resulted in the oldest divergence time estimates throughout Convolvulaceae and Solanaceae (Fig. 2a). The 95\% HPD for the age of the divergence between Convolvulaceae and Solanaceae was 97-130 Myr, the 95\% HPD for the age of the Convolvulaceae crown node was 94-129 Myr, the 95\% HPD for the age of the Solanaceae crown node was 67-99 Myr, and the 95\% HPD for the age of the Ipomoea crown node was 18-43 Myr. There was a 20.3-fold difference between the maximum inferred substitution rate (MPE) of $2.9^{*} 10^{-3}$ substitutions $\mathrm{Myr}^{-1}$ (on the Solanaceae stem branch), and the minimum inferred substitution rate of $1.4^{*} 10^{-4}$ substitutions $\mathrm{Myr}^{-1}$ (Fig. 2a and S1a). However, apart 
464

465

466

467

468

469

470

471

472

473

474

475

476

477

478

479

480

481

482

483

484

485

486

487

488

489

490

491

492

493

494

495

496

497

from the Solanaceae stem branch, inferred rates are within a range between $1.4^{*} 10^{-4}$ and $1.4 * 10^{-3}$ substitutions $\mathrm{Myr}^{-1}$, a 10-fold difference.

Calibration Strategy 2 resulted in younger divergence time estimates (Fig. 2b). The 95\% HPD for the age of the divergence between Convolvulaceae and Solanaceae was 89-129 Myr, the 95\% HPD for the age of the Convolvulaceae crown node was 86-126 Myr, the 95\% HPD for the age of the Solanaceae crown node was 64-94 Myr, and the 95\% HPD for the age of the Ipomoea crown node was 17-41 Myr. There was a 21.5-fold difference between the maximum inferred substitution rate of $3.2 * 10^{-3}$ substitutions $\mathrm{Myr}^{-1}$ (on the Solanaceae stem branch), and the minimum inferred substitution rate of $1.5^{*} 10^{-4}$ substitutions $\mathrm{Myr}^{-1}$ (Fig. $2 \mathrm{~b}$ and S1b). However, apart from the Solanaceae stem branch, inferred rates fall within a range between $1.5^{*} 10^{-4}$ and $1.5^{*} 10^{-3}$ substitutions $\mathrm{Myr}^{-1}$, a 10 -fold difference.

Calibration Strategy 3 resulted in the youngest divergence time estimates (Fig. 2). The 95\% HPD for the age of the divergence between Convolvulaceae and Solanaceae was 57-58 Myr, the 95\% HPD for the age of the Convolvulaceae crown node was 46-56 Myr, the 95\% HPD for the age of the Solanaceae crown node was 56-58 Myr, and the 95\% HPD for the age of the Ipomoea crown node was 10-21 Myr. There was a 59-fold difference between the maximum inferred substitution rate of $1.3 * 10^{-2}$ substitutions $\mathrm{Myr}^{-1}$ (on the Convolvulaceae stem branch), and the minimum inferred substitution rate of $2.1 * 10^{-4}$ substitutions $\mathrm{Myr}^{-1}$ (Fig. 2c and S1c). However, apart from the Convolvulaceae stem branch, inferred rates fall within a range between $1.5^{*} 10^{-4}$ and $3.5^{*} 10^{-3}$ substitutions $\mathrm{Myr}^{-1}$, a 16-fold difference.

Calibration Strategy 4 also resulted in younger divergence time estimates than Calibration Strategy 1 and 2 (Fig. 2). The 95\% HPD for the age of the divergence between Convolvulaceae and Solanaceae was 41-83 Myr, the 95\% HPD for the age of the Convolvulaceae crown node was 40-81 Myr, the 95\% HPD for the age of the Solanaceae crown node was 26-59 Myr, and the 95\% HPD for the age of the Ipomoea crown node was 8$25 \mathrm{Ma}$. There was a 17.9-fold difference between the maximum inferred substitution rate of $4.8^{*} 10^{-3}$ substitutions $\mathrm{Myr}^{-1}$ (on the Solanaceae stem branch), and the minimum inferred substitution rate of $2.7 * 10^{-4}$ substitutions $\mathrm{Myr}^{-1}$ (Fig. $2 \mathrm{~d}$ and S1d). However, apart from the Solanaceae stem branch, inferred rates fall within a range between $1.5^{*} 10^{-4}$ and $2.5^{*} 10^{-3}$ substitutions $\mathrm{Myr}^{-1}$, a 9.2-fold difference.

With a strict clock, Calibration Strategies 1-3 resulted in divergence time estimates that were very similar to each other, and to divergence time estimates with Calibration Strategy 1 and a UCLN relaxed clock (Fig. S2a-c; Fig. 2a). Inferred substitution rates were also very similar for Calibration Strategies 1-3 with a strict clock (Fig. S2a-c). With a strict 
clock, Calibration Strategy 4 resulted in markedly younger divergence time estimates than Calibration Strategies 1-3 with a strict clock and calibration Strategy 4 with a UCLN relaxed clock (Fig. S2d, Fig. 2d). For all four calibration strategies, 95\% HPDs were far narrower with a strict clock (Fig. 2, Fig. S2).

\subsection{Divergence time estimates within Ipomoea}

\subsubsection{Divergence time estimates within Ipomoea inferred in treePL with the optimum} smoothing value

When the optimum smoothing value of 0.01 was used to infer a nuclear timecalibrated phylogeny in treePL, the inferred crown ages for the New World and Old World Clades were approximately $29 \mathrm{Myr}$ and $28 \mathrm{Myr}$ respectively (Fig. 3a). The major clades A-E, and Astripomoea and close relatives, are inferred to have originated 16-23Ma, throughout the Early Miocene (Fig 3a). The Parana Clade and Coriaceous Sepal Clade, and the Batatas group are inferred to have originated 7.5-12.5 Ma, during the Late Miocene. In this timecalibrated phylogeny, there was a 3.279-fold difference between the minimum inferred substitution rate of $6.21 * 10^{-4}$ substitutions $\mathrm{Myr}^{-1}$, and the maximum inferred substitution rate of $2.036 * 10^{-3}$ substitutions Myr $^{-1}$ (Fig. 3a, Fig. S3a).

When the optimum smoothing value of 10000 was used to infer a chloroplast timecalibrated phylogeny in treePL, the inferred crown ages for the New World and Old World Clades were approximately 25.6 Myr and 21 Myr respectively (Fig. 3b). Clades A, B, D, E, and Astripomoea and close relatives, are inferred to have originated 13.5-19.1 Ma, throughout the Early and Middle Miocene. Clade $\mathrm{C}$ is inferred to have originated during the Late Miocene, 8.9 Ma (Fig. 3b). In this time-calibrated phylogeny, the same substitution rate of $1.81 * 10^{-4}$ substitutions $\mathrm{Myr}^{-1}$ was inferred for every branch (Fig. 3b).

These results from the nuclear and chloroplast time-calibrated phylogenies indicate that much of the morphological and species diversity within Ipomoea, briefly summarised in Figure $3 c-j$, originated before the end of the Miocene. However, the limited taxon sampling means that it is difficult to construct detailed or precise hypotheses about when and how frequently specific morphological traits evolved.

\subsubsection{Alternative divergence time estimates for the Ipomoea nuclear phylogeny}

Divergence times inferred in treePL with alternative smoothing values were similar to divergence times inferred with the optimum value, although in some cases they were somewhat younger (Fig. 4a, Fig. S4a-c). With higher smoothing values, the magnitude of among-branch-substitution-rate-variation decreased (Fig. S3a-c, Fig. S4a-c). 
For analyses in RevBayes, in the skeletal time-calibrated phylogeny inferred with a

532

533

534

535

536

537

538

539

540

541

542

543

544

545

546

547

548

549

550

551

552

553

554

555

556

557

558

559

560

561

562

563

564

strict clock, MPEs for the ages of major clades were similar to age estimates inferred in treePL (Fig. 4a, Fig. S5a). 95\% HPD intervals were on average 10.9\% of the MPE for the age each clade (Fig. 4a, Table S2), and the inferred substitution rate was similar to those inferred in treePL (Fig. S5a). In the three-taxon time-calibrated phylogenies inferred with a strict clock, MPEs for the ages of major clades were somewhat older than in the skeletal timecalibrated phylogeny and 95\% HPDs were on average 4.7\% of the MPE for the age of each clade (Fig. 4a, Table S2).

In the skeletal time-calibrated phylogenies inferred with a relaxed clock, MPEs for the ages of major clades were similar to age estimates inferred in treePL and to the skeletal timecalibrated phylogeny inferred with a strict clock. However, there was more variation in the relationship between these age estimates and other estimates, compared to those previously discussed, with inferred ages being either older or younger depending on the clade (Fig. 4a, Fig. S5b). 95\% HPD intervals were markedly wider than in RevBayes analyses with a strict clock, on average they were $60 \%$ of the MPE for the age of each clade (Fig. 4a, Table S2). The substitution rate and magnitude of among-branch-substitution-rate-variation was similar to that inferred in treePL with a lower smoothing value (Fig. S5b, Fig. S6a). In three-taxon time-calibrated phylogenies inferred with a relaxed clock, MPEs tended to be slightly older than MPEs inferred in either of the skeletal time-calibrated phylogenies. However, for the two oldest clades in the phylogeny, the New Word and Old World clades, MPEs were markedly younger than other age estimates inferred in treePL or RevBayes (Fig. 4a). 95\% HPD intervals were considerably wider compared to the skeletal time-calibrated phylogeny inferred with a relaxed clock. On average they were $114.1 \%$ of the MPE for the age of each clade (Fig. 4a, Table S2).

\subsubsection{Alternative divergence time estimates for the Ipomoea chloroplast phylogeny}

Divergence times inferred in treePL with a smoothing value of 100 were very similar to divergence times inferred with a smoothing value of 10000 (Fig. 4b, Fig. S4f). With a smoothing value of 0.01 or 1 , inferred divergence times were significantly older. This was especially the case for clades A-E, the majority of which were inferred to have originated during the earliest Miocene or Late Oligocene (Fig. 4b, Fig. S4d and e). With lower smoothing values, the magnitude of among-branch-substitution-rate-variation increased (Fig. S3d-f, Fig S4d-f). With the lowest smoothing value of 0.01, there was a 193-fold difference between the maximum inferred substitution rate of $3.86 * 10^{-4}$ substitutions $\mathrm{Myr}^{-1}$, and the minimum inferred substitution rate of $2 * 10^{-6}$ substitutions $\mathrm{Myr}^{-1}$. 

strict clock, MPEs for the ages of major clades were most similar to age estimates inferred in treePL with a smoothing value of 10000 (Fig. 4b, Fig. S5c). 95\% HPD intervals were

568

569

570

571

572

573

574

575

576

577

578

579

580

581

582

583

584

585

586

587

588

589

590

591

592

593

594

595

596

597

598 approximately $16 \%$ of the MPE for the age of each clade (Fig. 4b, Table S2). As with divergence times, the inferred substitution rate was also similar to when a smoothing value of 10000 was used in treePL (Fig. S5c). In three-taxon time calibrated phylogenies inferred with a strict clock, MPEs for the ages of major clades were similar to those inferred in the skeletal time-calibrated phylogeny (Fig. 4b). 95\% HPDs were slightly wider than in the skeletal timecalibrated phylogeny, approximately $19 \%$ of the MPE for each clade (Fig. 4b, Table S2).

In skeletal time-calibrated phylogenies inferred with a relaxed clock, MPEs for the ages of major clades tended to be slightly older than in either of the two types of timecalibrated phylogeny inferred with a strict clock (Fig. 4b, Fig. S5d). They were therefore also slightly closer to age estimates inferred in treePL with smoothing values of 0.01 and $1.95 \%$ HPDs were also markedly wider than RevBayes analyses with a strict clock, approximately $72 \%$ of the MPE for the age of each clade (Fig. 4b, Table S2). The substitution rate, and magnitude of among-branch-substitution-rate-variation was similar to the chloroplast timecalibrated phylogeny inferred in treePL with a smoothing value of 0.01 (Fig. S5d, Fig. S6b). In three-taxon time-calibrated phylogenies inferred with a relaxed clock, MPEs for the ages of major clades tended to be slightly older than MPEs inferred according to alternative methods in RevBayes (Fig. 4b). 95\% HPD intervals were also markedly wider, approximately $132.4 \%$ of the MPE for each clade (Fig. 4b, Table S2).

\subsection{Diversification parameter estimates within Ipomoea}

\subsubsection{Nuclear Phylogeny}

Inferred diversification parameters were similar, regardless of the smoothing value that was used to infer the time-calibrated phylogeny (Fig. 3a, Fig. S4a-c, Table 1). With the time-calibrated phylogeny inferred with the optimum smoothing value of 0.01 , a single shift in diversification parameters near the origin of a clade that contains the Parana Clade and Coriaceous Sepal Clade, has by far the highest posterior probability ( $p=0.9)$ (Fig. 3a). This diversification parameter shift leads to a 5.9-fold increase in the net diversification rate to 0.802 species $\mathrm{Myr}^{-1}$, compared to the background rate of 0.136 species $\mathrm{Myr}^{-1}$. This net diversification rate increase is caused by an increase in the inferred speciation rate from 0.147 , to 0.820 species $\mathrm{Myr}^{-1}$ (Table 1). The finding that there is a single shift in diversification parameters is supported by comparison of Bayes factors, the primary method of model comparison in a Bayesian framework. These comparisons provided strong support 
599

600

601

602

603

604

605

606

607

608

609

610

611

612

613

614

615

616

617

618

619

620

621

for a model with a single shift in diversification parameters, regardless of the smoothing value that was used to infer the time-calibrated phylogeny (Table S3).

\subsubsection{Chloroplast Phylogeny}

In the time-calibrated phylogeny inferred with the optimum smoothing value of 10000, a single shift in diversification parameters near the origin of the Parana Clade has the highest posterior probability ( $\mathrm{p}=0.79)$ (Fig. 3b). Two alternative patterns, in which there are shifts in diversification parameters on immediately ancestral branches, have significantly lower posterior probabilities (Fig. 3b). The most probable diversification parameter shift is inferred to lead to a 3.2-fold increase in net the diversification rate to 0.641 species $\mathrm{Myr}^{-1}$, compared to the background rate of 0.199 species $\mathrm{Myr}^{-1}$ (Table 1). This net diversification rate increase is caused by an increase in the speciation rate from 0.212 to 0.768 species $\mathrm{Myr}^{-1}$. With the time-calibrated phylogeny inferred with a smoothing value of 100 , inferred diversification parameters were very similar to when a smoothing value of 10000 was used (Fig. S4f, Table 1). With the time-calibrated phylogenies inferred with smoothing values of 1 and 0.01, no discrete shifts in diversification parameters were inferred (Fig. S5d and e, Table 1). These findings were supported by comparison of Bayes factors. For time-calibrated phylogenies inferred with smoothing values of 10000 and 100, there was strong support for a model with a single shift in diversification parameters. For time-calibrated phylogenies inferred with smoothing values of 1 and 0.01 , there was strong support for a model in which there were no discrete shifts in diversification parameters (Table S3).

Table 1. Diversification parameter estimates for Ipomoea inferred in BAMM using different time-calibrated phylogenies as input trees.

\begin{tabular}{|c|c|c|c|c|}
\hline Time-calibrated phylogeny & $\begin{array}{c}\text { Background } \\
\text { speciation rate } \\
\left(\text { species } \mathrm{Myr}^{-1}\right)\end{array}$ & $\begin{array}{c}\text { Background } \\
\text { extinction rate } \\
\left(\text { species } \mathrm{Myr}^{-1}\right)\end{array}$ & $\begin{array}{c}\text { Shift } \\
\text { speciation } \\
\text { rate* }^{*}(\text { species } \\
\left.\text { Myr }^{-1}\right)\end{array}$ & $\begin{array}{c}\text { Shift } \\
\text { extinction } \\
\text { rate* }^{*}(\text { species } \\
\left.\mathrm{Myr}^{-1}\right)\end{array}$ \\
\hline Nuclear, smoothing $=0.01$ & 0.147 & 0.0108 & 0.820 & 0.0176 \\
\hline Nuclear, smoothing $=1$ & 0.150 & 0.00942 & 0.878 & 0.0184 \\
\hline Nuclear, smoothing $=100$ & 0.150 & 0.00950 & 0.895 & 0.0182 \\
\hline Nuclear, smoothing $=10000$ & 0.150 & 0.00954 & 0.898 & 0.0190 \\
\hline Chloroplast, smoothing $=0.01$ & 0.153 & 0.0136 & --- & --- \\
\hline Chloroplast, smoothing $=1$ & 0.150 & 0.0123 & --- & --- \\
\hline Chloroplast, smoothing $=100$ & 0.212 & 0.0135 & 0.775 & 0.131 \\
\hline Chloroplast, smoothing $=10000$ & 0.212 & 0.0131 & 0.768 & 0.126 \\
\hline
\end{tabular}

622

623

624

625

626

* Shift speciation rate and Shift extinction rate refer to rates for clades within which there are significant shifts in diversification parameters compared to the background rate. The phylogenetic position of these rate shifts is indicated in Figure 2 and Figure S5.

\subsection{When did the sweet potato evolve?}




\subsubsection{When did the sweet potato diverge from its closest relative?}

In time-calibrated phylogenies for the Batatas group inferred in RevBayes with a UCLN relaxed clock, the divergence between I. batatas and I. trifida was inferred to have occurred at least 1.9Ma in the nuclear time-calibrated phylogeny (Fig. 5a), and at least 0.89Ma in the chloroplast time-calibrated phylogeny (Fig. 5b). In nuclear and chloroplast time-calibrated phylogenies inferred with alternative methodologies, the inferred divergence time between I. batatas and I. trifida was always inferred to have occurred at least $0.8 \mathrm{Ma}$ (Fig. S7, Fig. S8). These alternative methodologies make different assumptions about the magnitude and nature of among-branch-substitution-rate-variation. As such, each analysis makes different inferences about substitution rates on individual branches (Fig. 5, Fig. S7, Fig. S8). Regardless of this, in all cases the divergence between I. batatas and I. trifida is inferred to have occurred in pre-human times.

We performed two further analyses with our chloroplast dataset. Where we constrained the divergence between I. batatas and I. trifida to have occurred no more than 15 $\mathrm{Ka}$, but retained the point calibration of $6.2 \mathrm{Myr}$ at the root node - we inferred a greater than 200-fold increase in substitution rates for branches leading to I. batatas and I. trifida. As a result, substitution rates in this part of the time-calibrated phylogeny exceed $2.2 * 10^{-2}$ substitutions $\mathrm{Myr}^{-1}$ (Fig. 6a, Fig. S9). This value far exceeds any previously inferred substitution or mutation rate for chloroplast DNA. This result was consistent regardless of whether we used a UCLN relaxed clock (Fig. 6a) or a random local clock (Fig. S9).

Where we constrained the divergence between I. batatas and I. trifida to occur $15 \mathrm{Ka}$, but removed the point calibration at the root node, we inferred a substitution rate of $2.1 * 10^{-2}$ substitutions $\mathrm{Myr}^{-1}$ for the Batatas group (Fig. 6b). As before, this rate is far higher than any previously inferred substitution or mutation rate for chloroplast DNA. Taken together, these two additional analyses demonstrate that in order to infer a divergence time between $I$. batatas and I. trifida that occurred in human times, and that is consistent with the storage root of sweet potato having originated as a result of human domestication, a substitution rate would be required that far exceeds any known substitution or mutation rate for chloroplast DNA.

From our time-calibrated phylogenies for the entire of Ipomoea, we were also able to show that storage roots are likely to have evolved at least 30 times independently throughout Ipomoea, and that in each case the origin of the storage root occurred in pre-human times. This was the case regardless of the smoothing value used to infer the time-calibrated 
phylogeny (Table S4). This highlights the capacity for storage roots to evolve within

661 Ipomoea independently of human actions.

662

663

664

665

666

667

668

669

670

671

672

673

674

675

676

677

678

679

680

681

682

683

684

685

686

687

688

689

690

691

692

693

\subsubsection{When did diversity within the sweet potato evolve?}

With the time-calibrated phylogenies that contained all sampled accessions of $I$.

batatas and I. trifida, the divergences between most of the lineages within I. batatas occurred in pre-human times (Fig. 7). We interpret this as strong evidence that most of the diversity within I. batatas evolved in pre-human times. This result was consistent with both the nuclear (Fig. 7a) and chloroplast datasets (Fig. 7b), and for analyses inferred in treePL with both the optimum and alternative smoothing values, or in RevBayes with a strict clock or UCLN relaxed clock (Fig. S10, Fig. S11). These alternative methodologies make different assumptions about the magnitude and nature of among-branch-substitution-rate-variation. As such, each analysis makes different inferences about substitution rates on individual branches (Fig. 7, Fig. S10-12). Regardless of this, in all cases the divergences between different accessions of I. batatas and I. trifida are inferred to have occurred in pre-human times.

In RevBayes, we also inferred divergence times in three-taxon time-calibrated phylogenies. The divergence time estimates in these three-taxon time-calibrated phylogenies differed somewhat from those in which all taxa were sampled and HPDs were considerably wider. However, as before, the divergences between different accessions of I. batatas and $I$. trifida predominantly occurred in pre-human times (Table S5).

\section{DISCUSSION}

\subsection{Calibration uncertainty provides an uncertain basis for inferring the temporal} dynamics evolution in Ipomoea

Different combinations and interpretations of fossil calibrations provide an uncertain and conflicting framework for calibrating the phylogeny of Convolvulaceae and Solanaceae. As such, different calibration strategies led to major differences in inferred divergence times within the two families, including for the crown node of Ipomoea (Fig. 2). With respect to fossils within Solanaceae, this study focussed on the recently discovered Physalis infinemundi, and the implications of different assumptions about the maximum possible age of Solanaceae. We showed that making stronger assumptions that the Solanaceae stem node is significantly younger than $130 \mathrm{Myr}$ - a commonly used hard maximum for the origin of eudicots - led to significantly younger divergence time estimates. This was the case even when Physalis infinemundi was retained as a minimum constraint of 52Myr for the Solanoideae stem node. In Calibration Strategy 3, where we made the strongest assumption that the Solanaceae stem node was significantly younger than $130 \mathrm{Myr}$, age estimates were 
694

695

696

697

698

699

700

701

702

703

704

705

706

707

708

709

710

711

712

713

714

715

716

717

718

719

720

721

722

723

724

725

726

similar to those obtained in previous studies where significantly younger fossil calibrations were used (Särkinen et al. 2013; Magallón et al. 2015; Mitchell et al. 2016). As well as evaluating fossil calibrations within Solanaceae, we also constructed a time-calibrated phylogeny with a secondary calibration from Magallón et al. (2015). This led to younger divergence time estimates that were similar to Calibration Strategy 3.

It is impossible to make a definitive judgement about which calibration strategy is the most robust. Assuming its phylogenetic placement is correct, Physalis infinemundi does provide a minimum constraint for the Solanoideae stem node. However, it would be misleading to suggest we know a most probable age for the Solanoideae stem node in relation to the age of this fossil. Alternatively, it may be reasonable to suggest that the Solanaceae stem node must be younger than $130 \mathrm{Myr}$ - but the likely extent to which it is younger is also difficult to quantify. Further, the maximum constraint of $130 \mathrm{Ma}$ is derived from the observation that there is an absence of eudicot fossils in sediments older than this age, but a sudden appearance in younger sediments (Doyle et al. 1977). This maximum constraint is therefore based on the absence of evidence - a problematic basis upon which to construct assumptions.

The implications of different calibration strategies are also influenced by assumptions about among-branch-substitution-rate-variation. Note, for example, how the same calibration strategy can lead to significantly different divergence time estimates, depending on whether a UCLN relaxed clock or a strict clock is used (Fig. 2 and Fig. S2). Further, for the same molecular clock model, different calibration strategies lead to marked differences in inferred substitution rates (Fig. 2, Fig. S1, Fig. S2). Taken together, this highlights the tight interaction between fossil calibrations, assumptions about among-branch-substitution-ratevariation, inferred substitution rates, and inferred divergence times.

Despite the uncertainty presented here, we suggest that presenting our results in this way provides the most robust basis for interpreting divergence time estimates, and accounting for factors that may affect inferences in the future. We do not, by contrast, present a single time-calibrated phylogeny, because no single time-calibrated phylogeny fully accounts for all the uncertainty that is represented here. Despite this complexity, with careful interpretation we suggest these results can provide a basis for discussing the temporal dynamics of evolution in Ipomoea, and answering specific questions about the origin of I. batatas.

\subsection{Uncertainty about substitution rate variation within Ipomoea leads to further} uncertainty in divergence time estimates 
We did not implement any fossil calibrations at internal nodes within Ipomoea.

728 Therefore, whilst uncertainty about the absolute age of the entire of Ipomoea can result from

729

730

731

732

733

734

735

736

737

738

739

740

741

742

743

744

745

746

747

748

749

750

751

752

753

754

755

756

757

758

759

760 calibration uncertainty (summarised in Figure 2), uncertainty about the relative ages of different clades within Ipomoea cannot result from calibration uncertainty. Instead, it results predominantly from assumptions about substitution rates, and in some cases additional assumptions about relative clade ages (expressed by the birth-death branching process in Bayesian analyses). In our analyses of divergence times within Ipomoea, we therefore experimented with different assumptions about substitution rates, and in Bayesian analyses, with the assumptions of the birth-death branching process.

In nuclear time-calibrated phylogenies inferred in treePL, we showed that different smoothing values have a limited effect on inferred divergence times (Fig. 3a, Fig. 4a, Fig. S4a-c). Regardless of the smoothing value, the majority of major clades are inferred to have originated in the Late Oligocene and throughout the Early Miocene, and the Parana Clade, Coriaceous Sepal Clade, and the Batatas group are inferred to have originated in the Late Miocene. This implies that inferred divergence times within Ipomoea are fairly robust to assumptions about among-branch-substitution-rate-variation. This result is likely to be explained by the fact that regardless of the smoothing value, the magnitude of inferred among-branch-substitution-rate-variation was always fairly low (Fig. 3a, Fig. S3a-c, Fig. $\mathrm{S} 4 \mathrm{a}-\mathrm{c})$. Nonetheless, any conclusion about the robustness of these divergence time estimates assumes that the model of rate variation used in treePL - whereby substitution rates are correlated between ancestral and descendant branches (Sanderson 1997; Smith and O’Meara 2012) - is valid.

By contrast, in chloroplast time-calibrated phylogenies inferred in treePL, different smoothing values had a more profound effect on inferred divergence times. Specifically, lower smoothing values (0.01 and 1$)$ caused basal branches to be considerably shorter - and by consequence have considerably higher substitution rates - compared to higher smoothing values. This in turn meant that with lower smoothing values, inferred divergence times were considerably older such that the majority of major clades originated in the Late Oligocene or very early Miocene (Fig. 3b, Fig. 4b, Fig. S4d-f). Clearly, this result highlights that there is considerable uncertainty in divergence time estimates within Ipomoea. However, when experimenting with different smoothing values, we performed extensive cross-validation analyses to determine which smoothing values are most appropriate. For the chloroplast data, these suggested that higher smoothing values are more appropriate. Further, we suggest that divergence times inferred with higher smoothing values are more congruent with the nuclear 
time-calibrated phylogeny, with major clades originating throughout the Early Miocene, rather than exclusively in the very early Miocene. We therefore tentatively suggest that divergence times inferred with higher smoothing values are more accurate (Fig. 3b).

For analyses performed with nuclear data in RevBayes, the skeletal and three-taxon time-calibrated phylogenies were broadly congruent with those inferred in treePL (Fig. 4a, Fig. S5a and b). This provides further evidence that divergence time estimates with this dataset are relatively insensitive to assumptions about among-branch-substitution-ratevariation. However, we note that 95\% HPDs are considerably wider when a UCLN relaxed clock is used compared to a strict clock (Fig. 4a, Table S2). This highlights how uncertainty about substitution rates for individual branches - a source of uncertainty that is explicitly accounted for with a UCLN relaxed clock but not with a strict clock - leads to uncertainty in divergence time estimates. We also note that when a UCLN relaxed clock is used, 95\% HPD widths are considerably wider in three-taxon time calibrated phylogenies compared to skeletal time-calibrated phylogenies (Fig. 4a, Table S2). We suggest this reflects the fact that in the skeletal time-calibrated phylogenies, the birth-death branching process - which makes assumptions about divergence times - causes greater precision in divergence time estimates. This contrasts to the three-taxon time-calibrated phylogenies, where the birth-death branching process does make any assumptions about divergence times. This result has concerning implications because it suggests that the precision of divergence time estimates is sensitive to the assumption of the birth-death branching process that diversification parameters are constant. Given that this assumption is often likely to be violated, this precision may be misleading. Nonetheless, we tentatively suggest that the uncertainty expressed in the threetaxon time-calibrated phylogenies may be excessive given that all the time-calibrated phylogenies inferred in treePL corroborate with each other, and with the skeletal timecalibrated phylogenies inferred in RevBayes. However, we make this assertion whilst also considering the potential sources of error outlined above.

For analyses performed with chloroplast data in RevBayes, the skeletal and threetaxon time-calibrated phylogenies were broadly congruent with those inferred in treePL with smoothing values of 100 and 10000 (Fig. 4b, Fig. S5c and d). Given that smoothing values of 100 and 10000 are likely to be more appropriate for this dataset, and corroborate inferences derived from nuclear data, this result supports our previous inferences about when major clades within Ipomoea evolved (Fig. 3). Like with the nuclear data, 95\% HPDs were considerably wider when a UCLN relaxed clock was used, and when divergence times were 
inferred in three-taxon time-calibrated phylogenies (Fig. 4b, Table S2). We suggest the same explanations as in the discussion of nuclear time-calibrated phylogenies.

Taken together, these results provide tentative support that the majority of major clades within Ipomoea evolved in the Late Oligocene and throughout the Early Miocene, and the Parana Clade, Coriaceous Sepal Clade, and the Batatas group evolved in the Late Miocene. However, these conclusions are dependent on several important variables. These include the Calibration Strategy used to infer the time-calibrated phylogeny for Convolvulaceae and Solanaceae, the assumptions of different methods that account for among-branch-substitution-rate-variation, and - in Bayesian analyses - the assumption of a constant rate of speciation and extinction. It is important to consider the effect of these variables when interpreting the divergence time estimates presented here. Specifically, the calibration strategy will affect inferences of the absolute age of the entire of Ipomoea, whilst assumptions about the magnitude and nature of among-branch-substitution-rate-variation and the constancy of speciation and extinction rates, will affect inferences of the relative ages of different clades.

\subsection{Significant diversification parameter variation within Ipomoea is highly likely}

Inferences of diversification parameters are sensitive to uncertainty and error in divergence time estimates. In order to account for this, we inferred diversification parameters on time-calibrated phylogenies inferred with different smoothing values in treePL. With the nuclear time-calibrated phylogenies, we inferred a significant net diversification rate increase for the clade that contains both the Parana Clade and Coriaceous Sepal Clade. This net diversification rate increase was strongly supported, regardless of the smoothing value (Fig. 3a, Fig. S4a-c, Table 1). We were not surprised to infer a net diversification rate increase for this clade. Species within the Parana Clade and Coriaceous Sepal Clade often have very localised geographical ranges, contain groups of species whose morphologies overlap significantly, but overall, exhibit a variety of different growth forms and occur in a range of different habitats. These are characteristic attributes of rapid evolutionary radiations (Hughes and Eastwood. 2006; Givnish et al. 2009; Nevado et al. 2016).

With the chloroplast time-calibrated phylogenies inferred with smoothing values of 100 and 10000, we inferred a significant net diversification rate increase in the Parana Clade but not the Coriaceous Sepal Clade. By contrast, we did not infer any significant shifts in diversification parameters when a smoothing value of 0.01 or 1 was used (Fig. 3b, Fig. S4d-f, Table 1). However, given our cross-validation analyses suggest smoothing values of 100 and 10000 are more appropriate, we suggest the chloroplast dataset supports a significant net 
diversification rate increase at the origin of the Parana Clade. Taken together, the nuclear and chloroplast time-calibrated phylogenies provide strong support for a significant net diversification rate increase at either the origin of the clade containing the Parana Clade and Coriaceous Sepal Clade, or at the origin of the Parana Clade.

Despite the likely presence of net diversification rate shifts within Ipomoea, there is considerable uncertainty about the nature of these shifts. First, the possibility of erroneous divergence time estimates means there is considerable uncertainty about when the net diversification rate shifts actually occurred. Although we accounted for divergence time estimation error to an extent, by inferring diversification parameters on time-calibrated phylogenies inferred with different smoothing values, we did not account for other factors. For example, calibration uncertainty may mean that the entire phylogeny is calibrated to an incorrect timescale. A further factor that will likely affect the inferred timing and number of net diversification rate shifts, is the limited sampling within the phylogeny. For example, in the Parana Clade, only around 33\% of the species are sampled. Although we corrected for this in BAMM by specifying sampling proportions, a robust and well-resolved phylogeny that includes these missing species may result in the inference of several nested net diversification rate shifts that could not be recovered in the analyses presented here.

A further aspect of these net diversification rate shifts for which considerable uncertainty remains, is whether they reflect changes in the speciation rate or extinction rate. Our analyses in BAMM suggest they result from significant increases in the speciation rate (Table 1). However, in many cases it is likely to be very difficult to determine the relative contribution of speciation rate changes and extinction rate changes on inferred diversification dynamics (Rabosky 2010, 2014; Sanderson and Donoghue 2015; Moore et al. 2016). This is primarily because extinct branches are not sampled in a molecular phylogeny. Uncertainty about the relative importance of changes in speciation rates and changes in extinction rates will have profound implications for how hypothesis are constructed to explain the net diversification rate shifts discussed here. Further, a failure to sample extinct branches may mean that other aspects of the diversification history of Ipomoea have been entirely overlooked in this study.

\subsection{The sweet potato evolved in pre-human times}

We inferred time-calibrated phylogenies for Ipomoea in treePL using a point calibration for the root node that was equal to the MPE for the age of Ipomoea from Calibration Strategy 4. We suggest this calibration leads to significant underestimation of divergence times within Ipomoea, because the calibration strategy from which it is derived is 
862

863

864

865

866

867

868

869

870

871

872

873

874

875

876

877

878

879

880

881

882

883

884

885

886

887

888

889

890

891

892

893

894

895

likely to bias estimates to younger ages. Biasing our analysis in this way represents the most robust test of whether the storage root of I. batatas evolved in pre-human times. In these time-calibrated phylogenies, the divergence between $I$. batatas and its closest wild relative $I$. trifida was always in pre-human times. This was the case regardless of the smoothing value, or whether the phylogeny was inferred from nuclear or chloroplast data (Fig. S7a-d, Fig. S8ad). When we also inferred divergence times between $I$. batatas and its closest wild relatives in a Bayesian framework, the inferred divergence between I. batatas and I. trifida also significantly predated human times. This was the case regardless of whether a strict clock or a UCLN relaxed clock was used, or whether the phylogeny was inferred with nuclear or chloroplast data (Fig. 5, Fig. S7f, Fig. S8f). I. batatas is the only species amongst its close relatives that possesses a storage root, and together these results indicate that the storage root of I. batatas evolved in pre-human times.

We queried the conclusion that the divergence between I. batatas and I. trifida occurred in pre-human times by performing analyses with the chloroplast dataset where the divergence between I. batatas and I. trifida was constrained such that it occurred $15 \mathrm{Ka}$. In order to satisfy this calibration, we showed that the substitution rate for the chloroplast would be $2 * 10^{-2}$ substitutions $\mathrm{Myr}^{-1}$ (Fig. 6, Fig. S9). This is far higher than any previously recorded substitution or mutation rate for chloroplast DNA. Although there might be an expectation of elevated substitution rates for some genes in association with domestication, we would not expect such large rate differences to be observed across the entire plastome. Further, the 15 Ka constraint that we used is likely to represent an upper limit of when humans began domesticating crops, with most estimates for the origin of crop domestication being closer to $10 \mathrm{Ka}$ (Feldman 2001; Hillman et al. 2001; Zohary et al. 2006; Kingsbury 2009). To satisfy this younger timescale, such that the divergence between I. batatas and I trifida was constrained to have occurred $10 \mathrm{Ka}$, even higher substitution rates would be required than are recorded here.

The conclusion that the storage root of I. batatas evolved in pre-human times is consistent with the observation that storage roots are likely to have evolved repeatedly throughout Ipomoea, and each origin of the storage root in Ipomoea occurred in pre-human times (Table S4; Munoz et al. 2019). This indicates the capacity for storage roots to evolve in Ipomoea independently of human action.

We also showed that the majority of diversity within I. batatas is likely to have evolved in pre-human times. In our time-calibrated phylogenies constructed from all sampled accessions of $I$. batatas and I. trifida, divergence events between different accessions 
896

897

898

899

900

901

902

903

904

905

906

907

908

909

910

911

912

913

914

915

916

917

918

919

920

921

922

923

924

925

926

927

928

significantly predated human times (Fig. 7, Fig. S10-11, Table S5). This was the case regardless of the method used. These different methods included using treePL with different smoothing values, Bayesian analyses with a strict clock and UCLN relaxed clock, and Bayesian analyses with three-taxon time-calibrated phylogenies that removed temporal assumptions associated with the birth-death tree prior.

\subsection{Assimilating uncertainty and making conclusions}

It is challenging to assimilate the uncertainty in the results presented here. By highlighting the assumptions that underpin different methods, the different possibilities of potential uncertainty can appear immense. In most studies performed in a Bayesian framework, the $95 \%$ HPD is presented as an effective way of accounting for uncertainty. However, as demonstrated here, the 95\% HPD is entirely dependent on the assumptions that are made when performing an analysis. When the validity of different sets of assumptions is difficult to distinguish, as is often the case when inferring divergence times and diversification rates, no single 95\% HPD is therefore capable of reflecting the uncertainty inherent in these different assumptions. Thus, a more valuable approach for future studies, as followed here, may be to comprehensively account for the different types of assumptions that underlie inferences about the temporal dynamics of evolutionary diversification and openly discuss the implications of these. This may not provide simple answers to questions, but may provide a more robust foundation upon which future studies of macro-evolution can rest.

Despite the uncertainty we present here, we stress that when questions are carefully framed, and results carefully interpreted, novel and robust inferences about the temporal dynamics of evolutionary history can be made. In the analyses we present here, this includes the inference that the storage root of I. batatas evolved in pre-human times and was not a result of human domestication, and the inference that there is likely to be at least one significant shift in diversification rates within Ipomoea.

Regardless, in some cases it remains difficult to perform analyses from which robust inferences and confident conclusions about the temporal dynamics of evolutionary history can be made. Even when this is the case, for example with respect to the age of Ipomoea or major clades within Ipomoea, or the timing and frequency of diversification rate shifts, exploring the uncertainty in these inferences is invaluable. It can provide a basis upon which to develop new hypotheses that can be meaningfully tested with available data in the future, as well as providing a framework for prioritising the collection of new data. Further, it can stimulate theoretical and methodological studies that aim to characterise the extent to which 
929

930

931

932

933

934

935

936

937

938

939

940

941

942

943

944

945

946

947

948

949

950

951

952

953

954

955

956

957

958

959

960

961

962

963

964

965

966

967

968

969

970

971

972

inferences about macroevolutionary history can be made from analysing molecular phylogenies.

\section{FUNDING}

This work was supported by a NERC scholarship granted through the Environmental

Research DTP programme to TC.

\section{REFERENCES}

Baldwin BG and Sanderson MJ. 1998. Age and rate of diversification of the Hawaiian silversword alliance (Compositae). Proc Natl Acad Sci USA. 95:9402-9406.

Britton T. 2005. Estimating Divergence Times in Phylogenetic Trees Without a Molecular Clock. Syst. Biol. 54:500-507.

Bromham L. 2006. Molecular dates for the Cambrian Explosion: is the light at the end of the tunnel an oncoming train? Palaeontol Electron. 9:2004-2006.

Cardillo M, Weston PH, Reynolds ZKM, Olde PM, Mast AR, Lemmon EM, Lemmon AR, Bromham L. 2017. The phylogeny and biogeography of Hakea (Proteaceae) reveals the role of biome shifts in a continental plant radiation. Evolution. 71:1928-1943.

Castresana J. 2000. Selection of conserved blocks from multiple alignments for their use in phylogenetic analysis. Mol Biol Evol. 17:540-552.

Crisp MD, Arroyo MTK, Cook LG, Gandolfo MA, Jordan GJ, McGlone MS, Weston PH, Westoby M, Wilf P, Linder HP. 2009. Phylogenetic biome conservatism on a global scale. Nature. 458:754756.

Dexter KG, Lavin M, Torke BM, Twyford AD, Kursar TA, Coley PD, Drake C, Hollands R, Pennington RT. 2017. Dispersal assembly of rain forest tree communities across the Amazon basin. Proc. Natl. Acad. Sci. USA. 114:2645-2650.

Donoghue MJ. 2008. A phylogenetic perspective on the distribution of plant diversity. Proc Natl Acad Sci USA. 105:11549-11555.

Donoghue MJ and Sanderson MJ. 2015. Confluence, synnovation, and depauperons in plant diversification. New Phytol. 207:260-274.

Doyle J. A., Biens P., Dorenkamp A., Jardiné S. 1977. Angiosperm pollen from the pre-Albian Cretaceous of Equatorial Africa. Bull. Cent. Rech. Explor. 1:451-473.

Drummond AJ, Ho SYW, Phillips MJ, Rambaut A. 2006. Relaxed Phylogenetics and Dating with Confidence. PLOS Biol. 4:e88.

Drummond AJ and Suchard MA. 2010. Bayesian random local clocks, or one rate to rule them all. BMC Biol. 8:114.

Feldman M. 2001. Origin of Cultivated Wheat. pp. 3-58. in Bonjean AP. and Angus WJ. eds. The World Wheat Book: A History of Wheat Breeding. Lavoisier: Paris.

Fitch WM. 1976. Molecular evolutionary clocks. pp. 160-178 in Ayala FJ eds. Molecular Evolution. Sinauer: Sunderland. 
Folk RA, Stubbs RL, Mort ME, Cellinese N, Allen J, Soltis PS, Soltis DE, Guralnick RP. 2019. Rates of niche and phenotype evolution lag behind diversification in a temperate radiation. Proc Natl Acad Sci USA. 116: 10874-10882

977

Gillespie J. H. 1991. The Causes of Molecular Evolution. Oxford: Oxford University Press.

979 Givnish TJ, MIllam KC, Mast AR, Paterson TB, Theim TJ, Hipp AL, Henss JM, Smith JF, Wood

980 KR, Sytsma KJ. 2009. Origin, adaptive radiation and diversification of the Hawaiian lobeliads

981 (Asterales: Campanulaceae). Proc. R. Soc. B. 276:407-416.

982 Govindarajulu R, Hughes CE, Bailey CD. 2011. Phylogenetic and population genetic analyses of diploid Leucaena (Leguminosae; Mimosoideae) reveal cryptic species diversity and patterns of divergent allopatric speciation. Am. J. Bot. 98:2049-2063.

Hillman G, Hedges R, Moore A, Colledge S, Pettitt P. 2001. New evidence of Lateglacial ceresl cultivation in Abu Hureyra on the Euphrates. Holocene. 11:383-393.

987 Hohna S, Heath TH, Boussau B, Landis MJ, Ronquist F, Huelsenbeck JP. 2014. Probabilistic

988 Graphical Model Representation in Phylogenetics. Syst Biol. 63:753-771.

989 Hohna S, Landis MJ, Heath TA, Boussau B, Lartillot N, Moore BR, Huelsenbeck JP, Ronquist F.

2016. RevBayes: Bayesian Phylogenetic Inference Using Graphical Models and an Interactive ModelSpecification Language. Syst Biol. 65:726-736.

992 Huelsenbeck JP and Ronquist F. 2001. MRBAYES: Bayesian inference of phylogenetic trees.

993 Bioinformatics. 17:754-755.

994 Hughes E. and Eastwood R. 2006. Island radiation on a continental scale: Exceptional rates of plant 995 diversification after uplift of the Andes. Proc Natl Acad Sci USA. 103:10334-10339.

996 Hughes CE, Pennington RT, Antonelli A. 2013. Neotropical Plant Evolution: Assembling the Big 997 Picture. Bot. J. Linn. Soc. 171:1-18.

998 Katoh K. 2002. MAFFT: a novel method for rapid multiple sequence alignment based on fast Fourier 999 transform. Nucleic Acids Res. 30:3059-3066.

1000 Katoh K and Standley DM. 2013. MAFFT Multiple Sequence Alignment Software Version 7: 1001 Improvements in Performance and Usability. Mol Biol Evol. 30:772-780.

1002 Kingsbury N. 2009. Hybrid. The University of Chicago Press: Chicago.

1003 Koenan EJM, de Vos JM, Aitchison GW, Simon MF, Schrire BD, de Sousa ER, de Queiroz LP, 1004 Hughes CE. 2013. Exploring the tempo of species diversification in legumes. S. Afr. J. Bot. 89:19-30.

Lavin M and Pennington RT. 2015. The contrasting nature of woody plant species in different

1007 Lavin M, Schrire BP, Lewis G, Pennington RT, Delgado-Salinas A, Thulin M, Hughes CE, Matos explains geographically structured phylogenies in legumes. Philos. Trans. Royal Soc. 359:1509-1522.

1010 Magallón S, Gómez-Acevedo S, Sánchez-Reyes LL, Hernández-Hernández T. 2015. A Phytol. 207:437-453.

Magallón S, Hilu KW, Quandt D. 2013. Land plant evolutionary timeline: Gene effects are secondary to fossil constraints in relaxed clock estimation of age and substitution rates. Am J Bot. 100:556-573. 
Mitchell TC, Williams BRM, Wood JRI, Harris DJ, Scotland RW, Carine MA. 2016. How the temperate world was colonised by bindweeds: biogeography of the Convolvuleae (Convolvulaceae). BMC Evol. Biol. 16:16.

Moore BR, Höhna S, May MR, Rannala B, Huelsenbeck JP. 2016. Critically evaluating the theory and performance of Bayesian analysis of macroevolutionary mixtures. Proc. Natl. Acad. Sci. USA. 113:9569-9574.

Morris JL, Puttick MN, Clark JW, Edwards D, Kenrick P, Pressel S, Wellman CH, Yang Z, Schneider H, Donoghue PCJ. 2018. The timescale of early land plant evolution. Proc Natl Acad Sci USA. 115:E2274-E2283.

Muñoz-Rodríguez P, Carruthers T, Wood JRI, Williams BRM, Weitemier K, Kronmiller B, Ellis D, Anglin NL, Longway L, Harris SA, Rausher MD, Kelly S, Liston A, Scotland RW. 2018. Reconciling Conflicting Phylogenies in the Origin of Sweet Potato and Dispersal to Polynesia. 28:1246-1256.

Muñoz-Rodríguez P, Carruthers T, Wood JRI, Williams BRM, Weitemier K, Kronmiller B, Goodwin Z, Sumadijaya A, Anglin NL, Filer D, Harris D, Rausher MD, Kelly S, Liston A, Scotland RW. 2019. Assessing the biodiversity of morning glories at different scales using global natural history collections. Nat Plants, in press.

Nevado B, Atchison GW, Hughes CE, Filatov DA. 2016. Widespread adaptive evolution during repeated evolutionary radiations in New World lupins. Nat. Commun. 7:1-9.

Pennington RT, Lavin M, Oliveira-Filho A. 2009. Woody Plant Diversity, Evolution, and Ecology in the Tropics: Perspectives from Seasonally Dry Tropical Forests. 40:437-457. Annu. Rev. Ecol. Evol. Syst. 40:437-457.

Rabosky DL. 2014. Automatic detection of key innovations, rate shifts, and diversity-dependence on phylogenetic trees. PLOS ONE 9:e89543.

Rabosky DL, Chang J, Title OP, Cowmann PF, Sallan L, Friedman M, Kaschner K, Garilao C, Near TJ, Coll M, Alfaro ME. 2018. An inverse latitudinal gradient in speciation rate for marine fishes. Nature. 559:392-395.

Rabosky DL, Lovette IJ. 2008. Explosive Evolutionary Radiations: Decreasing Speciation or Increasing Extinction Through Time? Evolution. 62:1866-1875.

Rambaut A, Suchard MA, Xie D, Drummond AJ. 2014. Tracer v1.6 Available at: http://beast.bio.ed.ac.uk/Tracer

dos Reis M. and Yang Z. 2013. The unbearable uncertainty of Bayesian divergence time estimation. $J$. Syst. Evol. 51:30-43.

Ronquist F, Teslenko M, Van Der Mark P, Ayres DL, Darling A, Höhna S, Larget B, Liang L, Suchard MA, Huelsenbeck JP. 2012. MrBayes 3.2: Efficient Bayesian Phylogenetic Inference and Model Choice Across a Large Model Space. Syst Biol. 61:539-542.

Sanderson MJ. 1997. A Nonparametric Approach to Estimating Divergence Times in the Absence of Rate Constancy. Mol Biol Evol. 14:1218-1231.

Sanderson MJ and Doyle JA. 2001. Sources of error and confidence intervals in estimating the age of angiosperms from $r b c L$ and 18S rDNA data. Am. J. Bot. 88:1499-1516.

Särkinen T, Pennington RT, Lavin M, Simon MF, Hughes CE. 2012. Evolutionary islands in the Andes: persistence and isolation explain high endemism in Andean dry tropical forests. J Biogeogr. 39:884-900. 
Särkinen T, Bohs L, Olmstead RG, Knapp S. 2013. A phylogenetic framework for evoluitonary study of the nightshades (Solanaceae): a dated 1000 tip tree. BMC Evol Biol. 13:214.

1067 Simon MF, Grether R, de Queiroz LP, Skema C, Pennington RT, Hughes CE. 2009. Recent assembly 1068 of the Cerrado, a neotropical plant diversity hotspot, by in situ evolution of adaptations to fire. Proc.

1069 Natl. Acad. Sci. USA. 106:20359-20364.

1070 Simoes AR, Culham A, Carine M. 2015. Resolving the unresolved tribe: a molecular phylogenetic framework for the Merremieae (Convolvulaceae). Bot. J. Linn. Soc. 179:374-387.

1072 Smith SA and O'Meara BC. 2012. treePL: divergence time estimation using penalized likelihood for 1073 large phylogenies. Bioinformatics. 28:2689-2690.

1074 Srivastava G, Mehrotra RC, Dilcher DL. 2018. Paleocene Ipomoea (Convolvulaceae) from India with 1075 implications for an East Gondwana origin of Convolvulaceae. Proc Natl Acad Sci USA. 115:602810766033.

Stefanović S, Austin DF, Olmstead RG. 2003. Classification of Convolvulaceae: A Phylogenetic Approach. Syst. Biol. 28:791-806.

1080 Talavera G and Castresana J. 2007. Improvement of Phylogenies after Removing Divergent and 1081 Ambiguously Aligned Blocks from Protein Sequence Alignments. Syst Biol. 56:564-577.

1082 Thorne JL, Kishino H, Painter IS. 1998. Estimating the rate of evolution of the rate of molecular 1083 evolution. Mol Biol Evol. 15:1647-1657.

1092 Wilf P. and Escapa IH. 2015. Green Web or megabiased clock? Plant fossils from Gondwanan

Vaidya G, Lohman DJ, Meier R. 2011. SequenceMatrix: concatenation software for the fast assembly of multi-gene datasets with character set and codon information. Cladistics. 27:171-180.

Warnock RCM, Parham JF, Joyce WG, Tyler R, Donoghue PCJ. 2015. Calibration uncertainty in molecular dating analyses: there is no substitute for the prior evaluation of time priors. Proc $R$ Sol B. 282:20141013.

Wilf P, Carvalho MR, Gandalfo MA, Ruben Cuneo N. 2017. Eocene lantern fruits from Gondwanan Patagonia and the early origins of Solanaceae. Science. 355:71-75.

1094 Zohary D, Hopf M, Weiss E. 2006. Domestication of Plants in the Old World. Oxford University 1095 Press: Oxford.

1096 Zuckerkandl E. and Pauling LB. 1962. Molecular disease, evolution, and genetic heterogeneity. pp. 1097 189-225. in Kasha M. and Pullman B. eds. Horizons in biochemistry. Academic Press: New York.

1098 Zuckerkandl E. and Pauling L. 1965. Evolutionary Divergence and Convergence. pp. 97-166 in 1099 Bryson V. and Vogel HJ. eds. Evolving genes and proteins. Academic Press: New York.

1100 Zhu T., dos Reis M., Yang Z. 2015. Characterization of the Uncertainty of Divergence Time 1101 Estimation under Relaxed Molecular Clock Models Using Multiple Loci. Syst. Biol. 64:267-280.

\section{FIGURE CAPTIONS}

Figure 1. A summary of analyses performed in this study. The dark grey boxes in the centre of the figure illustrate the progression from a sequence alignment, to a phylogeny, to a timecalibrated phylogeny, to inferring diversification parameters. The light grey boxes summarise the assumptions and issues associated with each step, and the way in which they were accounted for in this study. 
1112 Figure 2. Time-calibrated phylogenies for Convolvulaceae and Solanaceae inferred with different calibration strategies and a UCLN relaxed clock. Branch colours refer to the inferred substitution rate (MPE). Black circles refer to calibrated nodes. The grey " 1 " refers to the Solanaceae stem node (divergence between Solanaceae and Convolvulaceae), the grey " 2 " refers to the Convolvulaceae crown node, the grey " 3 " refers to the Solanaceae crown node, the grey " 4 " refers to the Ipomoea crown node. Grey bars refer to 95\% HPD intervals for the ages of these nodes. The time axis includes the geological period: $\mathrm{K}=$ Cretaceous, $\mathrm{P}=$ Paleogene, $\mathrm{Ng}=$ Neogene, $\mathrm{Q}=$ Quaternary. a) Calibration Strategy 1 - the Solanaceae stem node has a maximum age constraint of 130Myr, and the Solanoideae stem node has a minimum age constraint of 52Myr. b) Calibration Strategy 2 - the Solanaceae stem node is calibrated with a lognormal distribution with offset $=52, m=10.4, v=10.4$. The Solanoideae stem node has a minimum age constraint of 52Myr. c) Calibration Strategy $3-$ the Solanaceae stem node is calibrated with a lognormal distribution with offset $=52, m=5.2, v$ $=2.1$. The Solanoideae stem node has a minimum age constraint of 52Myr. d) The Solanaceae stem node is calibrated with a secondary calibration from Magallon et al. (2015). This is implemented as a normal distribution with $\mu=67.34, \sigma=9.980$.

Figure 3. Time-calibrated phylogenies for Ipomoea inferred in treePL with the optimum smoothing value, and images of species from different clades. A point calibration of 30.3 Myr is used at the root node in both a) and b). This is the MPE for the age of Ipomoea from Calibration Strategy 1. Where rates differ between branches, branch colours refer to the inferred substitution rate. Labelled nodes refer to nodes defining major clades. A - E refer to clades A - E from Muñoz-Rodríguez et al. (2019), IA refers to Astripmoea and close relatives, NW and OW refer to the New World and Old World clades (see Muñoz-Rodríguez et al. 2019), par refers to the Parana Clade, cs refers to the Coriaceous Sepal Clade, and bat refers to the Batatas group. Labelled arrows refer to the positions of shifts in diversification parameters inferred in BAMM. The number next to each arrow refers to the posterior probability of the shift occurring at that position in the phylogeny. Only shifts with a posterior probability of $>0.05$ are indicated. The time axis includes the geological epoch: $\mathrm{Ol}$ = Oligocene, $\mathrm{M}=$ Miocene, $\mathrm{P}=$ Pliocene, $\mathrm{Ps}=$ Pleistocene, and $\mathrm{H}=$ Holocene . a) shows a nuclear time-calibrated phylogeny, and b) shows a chloroplast time-calibrated phylogeny. $\mathbf{c}$ ) I. cordatotriloba, a trailing and twining species in the Batatas group. d) I. nil, a twining species in clade B. e) I. cairica, a trailing and twining species in clade E. f) I. bonariensis, a trailing and twining species in the Coriaceous Sepal Clade. g) I. paraguariensis, an erect species in the Parana clade. h) I. paludosa, an erect species in the Parana clade. i. $I$. psammophila, a trailing species in the Parana clade. j) I. megapotamica, a trailing and climbing species in the Parana clade.

Figure 4. Inferred ages for major clades within Ipomoea in nuclear a) and chloroplast b) time-calibrated phylogenies. Major clades and geological periods are labelled as set out in Figure 3. Different coloured points refer to age estimates derived from different inference methods (see key). For treePL these age estimates refer to point age estimates, for RevBayes these age estimates refer to MPEs. Where RevBayes has been used, the grey bar refers to the 95\% HPD.

Figure 5. Time-calibrated phylogenies for the Batatas group inferred in RevBayes with a UCLN relaxed clock. Branch colours refer to the inferred substitution rate. The grey bar refers to the 95\% HPD for the divergence between I. batatas and I. trifida. a) shows the nuclear time-calibrated phylogeny, b) shows the chloroplast time-calibrated phylogeny. 
1162 Figure 6. Alternative chloroplast time-calibrated phylogenies for the Batatas group inferred in RevBayes. Where rates differ between branches, branch colours refer to the inferred substitution rate. In a) the divergence between I. batatas and I. trifida is calibrated to $15 \mathrm{Kyr}$ and the root node has a point calibration of $6.18 \mathrm{Myr}$. This is the same root calibration as was used in all the previous time-calibrated phylogenies for the Batatas group inferred in RevBayes. A UCLN relaxed clock is used to infer substitution rates. In b) the divergence between I. batatas and I. trifida is constrained to have occurred $15 \mathrm{Ka}$ and there is no calibration at the root node. A strict clock is used to infer substitution rates.

Figure 7. Time-calibrated phylogenies for I. batatas and I. trifida inferred in treePL with the optimum smoothing value. All sampled accessions of the two species are included. Branch colours refer to the inferred substitution rate. a) shows the nuclear time-calibrated phylogeny, b) shows the chloroplast time-calibrated phylogeny. 
1 The temporal dynamics of evolutionary diversification in Ipomoea

2 Tom Carruthers ${ }^{1}$, Pablo Muñoz-Rodríguez ${ }^{1}$, John R. I. Wood ${ }^{1}$, and Robert W.

3 Scotland $^{1 *}$

4 Affiliations:

$5{ }^{1}$ Department of Plant Sciences, University of Oxford, South Parks Road, Oxford, OX1 3RB,

6 United Kingdom

$7 \quad$ * Correspondence to be sent to: Department of Plant Sciences, University of Oxford, South

8 Parks Road, Oxford, OX1 3RB, United Kingdom; E-mail: robert.scotland@plants.ox.ac.uk

9

10

11

12

13

14

15

16

17

18

19

20

21

22

23

24

25

26

27

28 


\section{Abstract}

30 Molecular phylogenies are used as a basis for making inferences about macroevolutionary history. However, a robust phylogeny does not contain the information that is necessary to make many of these inferences. Complex methodologies that incorporate important assumptions about the nature of evolutionary history are therefore required. Here, we explore the implications of these assumptions for making inferences about the macroevolutionary history of Ipomoea - a large pantropical genus of flowering plants that contains the sweet potato (Ipomoea batatas), a crop of global economic importance. We focus on assumptions that underlie inferences of divergence times, and diversification parameters (speciation rates, extinction rates, and net diversification rates). These are among the most fundamental variables in macroevolutionary research. We use a series of novel approaches to explore the implications of these assumptions for inferring the age of Ipomoea, the ages of major clades within Ipomoea, whether there are significant differences in diversification parameters among clades within Ipomoea, and whether the storage root of I. batatas evolved in pre-human times. We show that inferring an age estimate for Ipomoea and major clades within Ipomoea is highly problematic. Inferred divergence times are sensitive to uncertain fossil calibrations and differing assumptions about among-branch-substitution-rate-variation. Despite this uncertainty, we are able to make robust inferences about patterns of variation in diversification parameters within Ipomoea, and that the storage root of I. batatas evolved in pre-human times. Taken together, this study presents novel and generalizable insights into the implications of methodological assumptions for making inferences about macroevolutionary history. Further, by presenting novel findings relating to the temporal dynamics of evolution in Ipomoea, as well as more specifically to I. batatas, this study makes a valuable contribution to our understanding of tropical plant evolution, and the evolutionary context in which economically important crops evolve.

Key words: phylogenetics, evolution, divergence times, diversification, Ipomoea, Sweet Potato 


\section{INTRODUCTION}

Molecular phylogenies are of central importance to macroevolutionary research. Our understanding of evolutionary radiations, and more generally, the factors that affect when, how, and why diversity accumulates in different clades, is underpinned by the interpretation of molecular phylogenies in an evolutionary context (Baldwin and Sanderson 1998; Hughes and Eastwood 2006; Givnish et al. 2009; Rabosky et al. 2008; Koenan et al. 2013; Donoghue and Sanderson 2015; Nevado et al. 2016; Rabosky et al. 2018). So too is our understanding of when and how the earth's major biomes were assembled (Pennington et al. 2009; Simon et al. 2009; Govindarajulu et al. 2011; Särkinen et al. 2012; Hughes et al. 2013; Pennington and Lavin 2015; Dexter et al. 2017) and the historical processes that have shaped distribution patterns such as long-distance-dispersal and geological vicariance (Lavin et al. 2004; Donoghue 2008; Crisp et al. 2011; Muñoz-Rodríguez et al. 2018). Because of their importance to macroevolutionary research, the rapid increase in availability of robust molecular phylogenies for many groups has enabled macroevolutionary questions to be investigated in greater detail and a greater variety of different contexts.

There are fundamental differences between a robust molecular phylogeny and the evolutionary process that it is used as a basis for making inferences about. These differences mean that even if a robust phylogeny is known for a group, parameters of interest in macroevolutionary research such as divergence times, net diversification rates, speciation rates, and extinction rates can be inferred with significant error (Sanderson and Doyle 2001; Britton 2005; Rabosky 2010; Moore et al. 2016; Wilf and Escapa 2015; Carruthers et al. 2019). It is crucial to account for this error, especially when using inferences as a basis for constructing narratives or explanations about when, how and why a group evolved. If error is not adequately accounted for, complex narratives may be constructed that are not supported by available evidence (Wilf and Escapa 2015).

Here, we use the recently developed phylogenetic framework for Ipomoea (MuñozRodríguez et al. 2019) to investigate the temporal dynamics of its evolutionary diversification. Ipomoea is a pantropical genus of over 800 species that exhibit a range of growth forms including climbers, trailers, erect shrubs and trees. They also occur in a range of habitats including cerrado, dry forest, and disturbed wasteland (Wood et al. 2015). Further, the genus contains the sweet potato (I. batatas), a crop of global economic importance (Muñoz-Rodríguez et al. 2018). Investigating the temporal dynamics of evolutionary diversification in Ipomoea can therefore provide critical insights into the nature of 
evolutionary diversification in large tropical groups, and when, how and why important crops evolve from their wild relatives.

In this study, we specifically focus on the inference of divergence times, net diversification rates, speciation rates, and extinction rates. Net diversification rates, speciation rates, and extinction rates are subsequently referred to collectively as diversification parameters. We focus on divergence times and diversification parameters because these two variables are the fundamental basis upon which other aspects of macroevolutionary research depend. When inferring these two variables, we explicitly consider different sources of uncertainty. With respect to divergence times, one of the primary sources of uncertainty are fossil calibrations (Sanderson and Doyle 2001; dos Reis and Yang 2013; Magallón et al 2013; Warnock et al. 2015; Morris et al. 2018). These are likely to be a particularly important source of uncertainty for Ipomoea, and many other angiosperm genera, because their fossil records are extremely fragmentary (Särkinen et al. 2013; Mitchel et al. 2016; Cardillo et al. 2017; Wilf et al. 2017; Folk et al 2019). It is therefore difficult to make robust interpretations of the temporal signal in the fossil record, and to use it as a basis to calibrate phylogenies. For divergence times, we also consider the effect of different assumptions about substitution rates and the magnitude of among-branchsubstitution-rate-variation (Zuckerkandl and Pauling 1962, 1965; Fitch 1976; Gillespie 1991; Sanderson 1997; Thorne et al. 1998; Britton 2005; Bromham 2006; Drummond et al. 2006; Zhu et al. 2015; Carruthers et al. 2019), as well as the effect of assuming constant diversification parameters when performing divergence time analyses in a Bayesian framework. For the inference of diversification parameters, we explore the effect of divergence time estimation error on inferred diversification parameters. We also discuss the effects of limited taxon sampling, and the effects of extinct branches that are un-sampled in a molecular phylogeny.

When exploring these sources of uncertainty in the context of Ipomoea, we investigate several questions that relate to the timing and tempo of evolutionary diversification in the entire genus. These include the age of Ipomoea, the ages of major clades within Ipomoea, diversification parameters within Ipomoea, and the extent to which diversification parameters vary among clades within Ipomoea. Given the recently developed phylogenetic framework for Ipomoea resolves the relationships between I. batatas and its closest wild relatives (Muñoz-Rodríguez et al. 2018), we also investigate questions relating to the origin of I. batatas. This includes when I. batatas and its storage root evolved, and 
specifically, whether it evolved in pre-human times, or whether it evolved more recently as a likely result of human domestication.

\section{MATERIALS AND METHODS}

\subsection{Estimating the age of Ipomoea}

To estimate the age of Ipomoea, we inferred a time-calibrated phylogeny for Convolvulaceae, which contains Ipomoea, and its sister family Solanaceae, which has a richer fossil record (Särkinen et al. 2013; Wilf et al 2017). This time-calibrated phylogeny was based on a molecular dataset of 4 chloroplast genes $(a t p B, m a t K, n d h F, r b c L)$, the chloroplast intergenic spacer $\operatorname{trnL-trnF}$, and the nuclear ITS marker. Apart from sampled species of Ipomoea - for which we used sequences from Muñoz-Rodríguez et al. 2019, sampled sequences were downloaded from GenBank (Table S1). A total of 56 taxa were sampled to represent the phylogenetic breadth of the two families. We purposefully sampled a low proportion of taxa, and an equal number of taxa from each sister family. The rationale for this was to reduce the effect of violating the assumption of the constant rate birth-death branching process that the diversification parameters are constant - an underlying assumption of most Bayesian methods of divergence time estimation. This is because sampling an equal number of taxa from each sister clade will likely cause diversification parameters to be constant amongst sampled taxa at broad sales across the phylogeny, whilst sampling a small number of taxa will mean that any violation of the assumption of constant diversification parameters amongst sampled taxa will have a statistically weaker effect on divergence time estimates.

We aligned sequences in MAFFT v7.271 (Katoh 2002; Katoh and Standley 2013) using the L-INS-I setting and a gap opening penalty of 1.53. Ambiguously aligned regions were removed using default settings in Gblocks (Castresana 2000; Talavera and Castresana 2007), and Sequence Matrix v1.8 (Vaidya et al. 2011) was used to concatenate aligned sequences. The final concatenated matrix had 5,166 sites for the 56 taxa. We used MrBayes v3.2.6 (Huelesenbeck and Ronquist 2001; Ronquist et al. 2012) to infer the tree topology for subsequent divergence time analyses. A GTR + G + I model was used. This analysis was run for 2,000,000 generations and the final split frequency between runs was $<0.01$. The topology we recovered is consistent with previous phylogenetic studies of these two families (Stefanović et al. 2003; Särkinen et al. 2013; Simoes et al. 2015; Mitchell et al. 2016).

The validity of different temporal calibrations for Convolvulaceae and Solanaceae is unclear. We therefore experimented with different calibration strategies. However, in no case did we use any fossil calibrations within Ipomoea because there are no fossils that can 
reliably be assigned to the genus. This includes the recently published leaf fossils of Ipomoea meghalayensis (Srivastava et al. 2018). This is because the short thick petioles of these fossils are not typical of Ipomoea; their well-preserved gland dots, leaf venation, and cordate leaves are characteristic of several other genera; and from the fossils alone it cannot be determined that the plant has alternate leaves, as would be expected for Ipomoea (Hawthorne pers. comm.)

In Calibration Strategy 1, 2 and 3, the recently discovered fossil, Physalis infinemundi (Wilf et al. 2017) was used as a basis for implementing fossil calibrations. This fossil - dated at around 52 million years (Myr) - is far older than fossil calibrations previously used in Convolvulaceae and Solanaceae, and is also considerably older than previous age estimates for either of these two families (Särkinen et al. 2013; Magallón et al. 2015; Mitchell et al. 2016). Because of this, it places previous assumptions about the timing of evolution within these clades, and the relationship between fossil ages and clade ages, into considerable doubt.

In Calibration Strategy 1, both the Solanoideae stem node and Solanaceae stem node (root node) are calibrated with a uniform distribution $(\mathrm{min}=52$ million years ago $(\mathrm{Ma}), \max$ $=130 \mathrm{Ma}$ ). The minimum corresponds to the age of Physalis infinemundi, and the maximum corresponds to the time that eudicot pollen suddenly appears in the fossil record (Doyle et al 1977). This calibration strategy assumes the Solanoideae stem node is older than $52 \mathrm{Myr}$, and the Solanaceae stem node is younger than $130 \mathrm{Myr}$. Apart from this, these calibrations do not make explicit assumptions about where the most probable clade ages lie between the minimum and maximum constraints.

In Calibration Strategy 2 and 3, we accounted for the fact that $130 \mathrm{Myr}$ is most probably an overestimate of the Solanaceae stem node age - at least in the context of current understanding of the temporal dynamics of plant diversification (Särkinen et al. 2013; Magallón et al. 2015; Mitchell et al. 2016). We therefore calibrated the Solanaceae stem node with a lognormal calibration density parametrised as either: offset $=52, m=10.4, v=10.4$ (Calibration Strategy 2); or offset $=52, m=5.2, v=2.1$ (Calibration Strategy 3). In Calibration Strategies 2 and 3, the minimum constraint of $52 \mathrm{Ma}$ is retained at the Solanoideae stem node. The effect of Calibration Strategies 2 and 3 is therefore to shift the calibration density at the Solanaceae stem node to ages that are significantly younger than $130 \mathrm{Myr}$, with Calibration Strategy 3 shifting the distribution to younger ages than

\section{Calibration Strategy 2.}



most realistic, given that there is no evidence upon which to derive assumptions about how much younger than $130 \mathrm{Myr}$ the Solanaceae stem node is. Comparing these three Calibration Strategies therefore provides a basis to evaluate the implications of different assumptions about the likely age of the Solanaceae stem node.

In Calibration Strategy 4, we calibrated the Solanaceae stem node with a normal distribution $(\mu=67.34, \sigma=9.980)$. This is a secondary calibration from Magallón et al. (2015) in which 132 fossil calibrations were used throughout Spermatophyta. This calibration strategy is therefore not entirely dependent on the interpretation of fossil calibrations within Convolvulaceae and Solanaceae. Characteristics of the analysis from which Calibration Strategy 4 is derived (Magallón et al. 2015) are likely to bias estimates to younger ages. For example, this study implements an extremely young maximum constraint on the angiosperm crown age, and employs calibration densities throughout the phylogeny which assume that fossil ages are very close to clade ages. Therefore, age estimates derived from Calibration Strategy 4 were primarily used with respect to the evolution of the sweet potato. This is because biasing estimates to younger ages provided the most robust test of whether the storage root of the sweet potato evolved in pre-human times - one of the main hypotheses that we set out to examine.

We used RevBayes v1.0.4 (Hohna et al. 2014; Hohna, et al. 2016) to infer timecalibrated phylogenies according to the four calibration strategies. In all analyses, a GTR + G + I model was used with a birth-death branching process as the tree prior. The topology was fixed to that inferred in MrBayes, and starting trees consistent with the 4 different calibration strategies were generated in treePL (Smith and O’Meara 2012). In one set of analyses, a strict clock was used where the substitution rate was assumed to be the same for every branch. In a second set of analyses, an uncorrelated lognormal (UCLN) relaxed clock (Drummond et al. 2006) was used. For each analysis, two independent runs were performed. Sufficient mixing and convergence was assessed in Tracer v1.6.0 (Rambaut et al. 2014). A 25\% burn-in was used prior to calculating maximum a posteriori (MAP) trees, mean posterior estimates (MPEs), and 95\% highest posterior density intervals (HPDs).

\subsection{Inferring the ages of major clades within Ipomoea}

We used the mean posterior estimate (MPE) for the age of Ipomoea from Calibration Strategy 1 as a point calibration for the root node of more densely sampled phylogenies for the genus. We used this MPE because the calibration strategy from which it is derived does not make any explicit assumptions about probable clade ages in relation to minimum or 
maximum constraints. It can therefore act as a basis from which to discuss the implications of calibration strategies that make explicit assumptions about probable clade ages in relation to minimum or maximum constraints.

Because we do not use further fossil calibrations within Ipomoea, we can easily interpret the implications of root node calibrations for Ipomoea derived from different calibration strategies - the relative ages of nodes within the time-calibrated phylogeny will not change, only the absolute timescale over which the time-calibrated phylogeny occurs. This also explains why we use a point calibration for the root node, rather than a calibration density. We can easily compare the effects of root node calibrations that derive from different calibration strategies. By contrast, it would be difficult to construct a single calibration density that adequately accounts for uncertainty in root node estimates that derive from each of the calibration strategies outlined above.

For divergence time analyses within Ipomoea, we were interested in estimating the ages of major clades from Muñoz-Rodríguez et al. (2019); and also the Parana Clade - a diverse clade centred in central South America; the Coriaceous Sepal Clade - a diverse clade centred in the Caribbean; and the Batatas group - the clade of I. batatas and its closest wild relatives. We were also interested in constructing a temporal framework for subsequent analyses of diversification parameters.

For these divergence time analyses, we used the molecular datasets and phylogenies from Muñoz-Rodríguez et al. 2019. This includes a nuclear dataset of 434 single copy genes for 211 species of Ipomoea, and a plastome dataset of 206 species of Ipomoea. Within these more densely-sampled datasets, there may be significant differences in diversification parameters among clades. This violates a key assumption of Bayesian methods of divergence time estimation that use a constant rate birth-death branching process as the tree prior. We therefore used treePL to infer divergence times - a penalised likelihood method that does not use a constant rate birth-death branching process (Smith and O'Meara 2012). In treePL, we performed analyses with the nuclear and chloroplast phylogenies from Muñoz-Rodríguez et al. (2019) as input trees. Following extensive cross-validation, we used a smoothing value of 0.01 for the nuclear phylogeny, and 10000 for the chloroplast phylogeny (lower smoothing values assume more among-branch-substitution-rate-variation than higher smoothing values). For both phylogenies, we also inferred time-calibrated phylogenies with alternative smoothing values (1, 100 and 10000 for nuclear, and 0.01, 1 and 100 for chloroplast). By experimenting with different smoothing values, we were able to determine the sensitivity of 
divergence time estimates to different assumptions about the magnitude of among-branchsubstitution-rate-variation.

Inferred divergence times within Ipomoea may be sensitive to the unique characteristics of different methods - especially given there are no calibrations within Ipomoea with which to constrain age estimates. We therefore also used RevBayes to estimate divergence times within Ipomoea in two sets of analyses.

In one set of analyses, we constructed skeletal time-calibrated phylogenies for Ipomoea with the taxon-sampling designed such that the ages of clades of interest (set out above) were estimated. For the nuclear data, we sampled all genes that were sampled in every taxon in the skeletal phylogeny (36 taxa) and for which the aligned sequence length was at least 4,000 sites. For the chloroplast data, we sampled the entire plastomes for 37 taxa.

Divergence time estimates in the skeletal time-calibrated phylogenies may be biased by the tree prior which assumes constant diversification parameters when inferring divergence times. In the second set of analyses performed in RevBayes, a series of three taxon time-calibrated phylogenies was therefore inferred, the divergence times within which would not be affected by the assumptions of the tree prior. The taxa sampled within these three-taxon phylogenies was such that the root node of Ipomoea was always sampled, along with one further node from within Ipomoea that uniquely corresponded to a node within the skeletal phylogenies. Thus, for each node in the skeletal phylogenies, we generated two age estimates - one derived from the skeletal phylogeny itself, and one from the three-taxon phylogeny that sampled that node. For three-taxon phylogenies constructed from nuclear data, we sampled all genes sampled for all three species in each three-taxon phylogeny. For the chloroplast data, we sampled the entire plastomes for the three species sampled in each three-taxon phylogeny.

In both the skeletal and three-taxon time-calibrated phylogenies, the MPE for the age of Ipomoea from Calibration Strategy 1 was used as a point calibration for the root node. In both sets of analyses, we also used the same alignments as Muñoz-Rodríguez et al. (2019). For the skeletal phylogenies, the total aligned sequence length was 39,495 sites for the nuclear data, and 103,921 sites for the chloroplast data. We used a GTR + G + I model of sequence evolution, and both a strict clock and a UCLN relaxed clock. A birth-death process was used as the tree prior for the skeletal phylogenies. A Yule process was used as the tree prior for the three-taxon phylogenies. The Yule process was parameterised such that the prior probability for the age of the internal node - the single unknown divergence time - was uniformly distributed between the root age and the present. Divergence time estimates from 
the three-taxon phylogenies are not therefore biased by the birth-death branching process. In all analyses, the topologies were fixed such that they were congruent with those inferred by Muñoz-Rodríguez et al. (2019). Two independent runs were performed. Sufficient mixing and convergence was assessed in Tracer v1.6.0. A 25\% burn-in was used prior to calculating MAP trees, MPEs and 95\% HPDs.

\subsection{Inferring diversification parameters within Ipomoea}

We inferred diversification parameters in BAMM (Rabosky 2014). BAMM is a Bayesian method that determines the probability of different patterns of discrete shifts in diversification parameters occurring in a time-calibrated phylogeny. Current knowledge of diversification parameters within Ipomoea is very limited, whilst understanding of habit preferences, geographical distributions, and relationships amongst species is in some cases far from complete. Analyses of diversification parameters within Ipomoea were therefore exploratory. Nonetheless, one particular hypothesis we were interested in testing was whether the Parana Clade, which is very diverse and possesses species with overlapping morphologies and narrow distribution ranges, has significantly increased speciation rates.

We used the 8 time-calibrated phylogenies inferred in treePL (smoothing values of $0.01,1,100,10000$ with nuclear and chloroplast phylogenies) as input trees. We could therefore evaluate how different assumptions about among-branch-substitution-rate-variation (and by extension differences in divergence time estimates), as well as topological differences between the nuclear and chloroplast phylogenies, affect inferred diversification parameters. Following consultation with John Wood, who has carried out the majority of the taxonomic work in this project, we specified approximate sampling proportions for different clades. We used the set_priors.R script - downloaded with BAMM and requiring the $\mathrm{R}$ package BAMMtools - to select the appropriate priors. Four independent runs were performed for each analysis. For the chloroplast time-calibrated phylogeny inferred with a smoothing value of 0.01 , the analysis was run for 4,000,000 generations. For the chloroplast time-calibrated phylogeny inferred with a smoothing value of 1 , and the nuclear timecalibrated phylogeny inferred with a smoothing value of 0.01 , the analyses were run for $2,000,000$ generations. In all other cases, the analyses were run for 1,000,000 generations. A $10 \%$ burnin was used. Convergence was assessed using a custom R script that required the package BAMMtools. We compared Bayes factors between different models to identify the number of shifts in diversification parameters that occurred within our time-calibrated phylogenies. We then extracted the credible shift set - which represents the set of trees with 
different diversification parameter shift patterns that account for $95 \%$ of the posterior probability.

\subsection{When did the sweet potato evolve?}

To answer questions relating to the age of I. batatas, we used the MPE for the age of Ipomoea from Calibration Strategy 4 as a point calibration for the root node of timecalibrated phylogenies for Ipomoea. Calibration Strategy 4 does not account for the recently discovered and surprisingly old Physalis infinemundi. Further, several characteristics of the analysis (Magallón et al. 2015) from which Calibration Strategy 4 is derived are likely to mean that age estimates from this analysis are biased to younger ages. Age estimates derived from this calibration strategy are therefore likely to be considerably younger than those derived from Calibration Strategies 1 and 2 (and potentially 3). With respect to I. batatas, we are especially interested in determining whether its storage root evolved in pre-human times. Biasing our analysis to younger ages - by using Calibration Strategy 4 - is one of the most robust ways to do this, especially if, despite biasing analyses to younger ages, the recovered divergence times are pre-human.

We used this point calibration to calibrate the nuclear and chloroplast phylogenies for Ipomoea from Muñoz-Rodríguez et al. (2019). The time-calibrated phylogenies were inferred in treePL. The same optimum and alternative smoothing values were used as outlined previously.

The taxon sampling within these time-calibrated phylogenies is sufficient to estimate a divergence time between I. batatas and its closest wild relative I. trifida. Given I. trifida does not possess a storage root, the estimated divergence time between these two species can provide a basis for understanding when the storage root of I. batatas is likely to have evolved.

To scrutinise these divergence time estimates, we inferred additional time-calibrated phylogenies for the Batatas group in RevBayes. We could therefore explore the sensitivity of divergence time estimates in different methodological frameworks.

In an initial set of analyses in RevBayes, we used a point calibration for the root node (of the Batatas group) that was equal to the age estimate for the equivalent node in the nuclear time-calibrated phylogeny (for the entire genus) inferred with the optimum smoothing value in treePL. We inferred these phylogenies in RevBayes with both nuclear and chloroplast data from Muñoz-Rodríguez et al. (2018). For the nuclear data, we sampled all genes that were sampled in every species in the Batatas group. For the chloroplast data, we sampled the entire plastomes of every species in the Batatas group, except I. lactifera. The 
plastome of I. lactifera is highly reduced, and several problems resulted from its inclusion in preliminary analyses. We used the same alignments as Muñoz-Rodríguez et al. (2018). The total aligned sequence length was 375,628 sites for the nuclear data, and 160,435 sites for the chloroplast data. For both datasets, we used a GTR + G + I model of sequence evolution, and both a strict clock and a UCLN relaxed clock. We used a constant rate birth-death branching process as the tree prior. The topologies were fixed such that they were consistent with those inferred by Muñoz-Rodríguez et al. (2018). For each time-calibrated phylogeny, two independent runs were performed. Sufficient mixing and convergence was assessed in Tracer v1.6.0. A 25\% burn-in was used prior to calculating MAP trees, MPEs, and 95\% HPDs.

In a further set of analyses in RevBayes, the divergence between I. batatas and $I$. trifida was constrained such that it occurred $15 \mathrm{Ka}$. This is consistent with a timeframe that could allow for the storage root of I. batatas to be a product of domestication, given that the earliest evidence for crop domestication is a little over $10 \mathrm{Ka}$ (Feldman 2001; Hillman et al. 2001; Zohary et al. 2006; Kingsbury 2009). By using this constraint, we could infer the substitution rates required for the storage root of I. batatas to have evolved in human times as a result of domestication.

We performed two variants of this analysis. In the first, the root node of the Batatas group had the same point calibration as other analyses in this section. We therefore assume that the temporal framework inferred for the entire genus is broadly accurate. In the second, the root node did not have a point calibration. We therefore make no assumptions about the accuracy of the temporal framework inferred for the entire genus. This second variant is instead entirely independent of this temporal framework.

Where the root node had a point calibration, we expect our analysis may infer major differences in substitution rates between branches. As such we performed this analysis with both a UCLN relaxed clock - where substitution rates for each branch are drawn from a single continuous distribution, and a random local clock - where discrete (and potentially very large) changes in substitution rates between different branches are explicitly modelled (Drummond and Suchard 2011). Where the root node was not fixed, we used a strict clock. This additional set of analyses was performed with only the chloroplast data. As before, we used a GTR + G + I model of sequence evolution and a birth-death branching process as the tree prior. The topologies were fixed such that they were consistent with those inferred by Muñoz-Rodríguez et al. (2018). For each time-calibrated phylogeny, two independent runs were performed. Sufficient mixing and convergence was assessed in Tracer v1.6.0. A 25\% burn-in was used prior to calculating MAP trees, MPEs, 95\% HPDs. 

possess storage roots (Munoz et al. 2019). To provide further context for understanding the temporal dynamics of storage root evolution within Ipomoea, and determine whether there is

400

401

402

403

404

405

406

407

408

409

410

411

412

413

414

415

416

417

418

419

420

421

422

423

424

425

426

427

428

429

the capacity for them to evolve in pre-human times, we estimated when Ipomoea taxa with storage roots are likely to have diverged from relatives that do not possess storage roots. This provides a basis for understanding when storage roots are likely to have evolved throughout Ipomoea. These estimates were based on the nuclear time-calibrated phylogenies inferred in treePL with different smoothing values.

As well as estimating the divergence time between I. batatas and I. trifida, we also estimated the age of diversity within I. batatas. To do this, we inferred time-calibrated phylogenies with multiple accessions of I. batatas (71 accessions) and I. trifida (22 accessions). We inferred these time-calibrated phylogenies in treePL and RevBayes.

For analyses in treePL, we used nuclear and chloroplast phylogenies that contain multiple accessions of I. batatas and I. trifida (from Muñoz-Rodríguez et al. 2018) as input trees. We used a point calibration for the root node that was equal to the age estimate for the equivalent node in the nuclear time-calibrated phylogeny for the entire genus inferred with the optimum smoothing value in treePL. Following extensive cross-validation, we used a smoothing value of 0.01 for the nuclear and chloroplast phylogenies. We also inferred timecalibrated phylogenies with alternative smoothing values $(1,100,10000)$ to determine the sensitivity of divergence time estimates to different assumptions about the magnitude of among-branch-substitution-rate-variation.

In RevBayes, we performed two sets of analyses. First, we inferred time-calibrated phylogenies for all sampled accessions with either the nuclear or chloroplast data. For the nuclear data, we sampled the 40 genes that were sampled in every accession with the longest aligned sequence length. For the chloroplast data, we sampled entire plastomes. Second, we inferred a series of three-taxon time calibrated phylogenies with either the nuclear or chloroplast data. The motivation for this was the same as set out for the three-taxon timecalibrated phylogenies inferred across the whole of Ipomoea. The taxon sampling was such that in each three-taxon phylogeny, the root node of the phylogeny containing all sampled accessions was always sampled, along with one further node. For the nuclear data, we sampled all genes that were sampled for all three species in each three-taxon phylogeny. For the chloroplast data, we sampled the entire plastome for the three species sampled in each three-taxon phylogeny. We used the same alignments as Muñoz-Rodríguez et al. (2018). 
In all these analyses, a GTR + G + I model of sequence evolution was used. We used either a strict clock or a UCLN relaxed clock. We used a point calibration for the root node that was equal to the age estimate for the equivalent node in the nuclear time-calibrated phylogeny for the entire genus inferred with the optimum smoothing value in treePL. For the time-calibrated phylogenies containing all sampled accessions, a birth-death branching process was used as the tree prior. For the three-taxon time-calibrated phylogenies, a Yule process was used as the tree prior. This was parameterised such that the prior probability for the age of the internal node - the single unknown divergence time - was uniformly distributed between the root age and the present. In all analyses, the topologies were fixed such that they were consistent with those inferred by Muñoz-Rodríguez et al. 2018. For each time-calibrated phylogeny, two independent runs were performed. Sufficient mixing and convergence was assessed in Tracer v1.6.0. A 25\% burn-in was used prior to calculating MAP trees, MPEs and 95\% HPDs.

\subsection{A comprehensive analysis of uncertainty in our understanding of the temporal} dynamics of evolutionary diversification in Ipomoea

The purpose of the large array of analyses presented here is to comprehensively evaluate and account for the different sources of uncertainty when inferring divergence times, and subsequently, diversification parameters. These different sources of uncertainty are highly interlinked and complex. In Figure 1, we summarise these different sources of uncertainty, and the ways in which we have accounted for them in this study.

\section{RESULTS}

\subsection{Time-calibrated phylogenies for Solanaceae and Convolvulaceae, and an age} estimate for Ipomoea

Here, we summarise results when a UCLN relaxed clock is used with different calibration strategies for Convolvulaceae and Solanaceae, followed by results when using a strict clock.

Calibration Strategy 1 resulted in the oldest divergence time estimates throughout Convolvulaceae and Solanaceae (Fig. 2a). The 95\% HPD for the age of the divergence between Convolvulaceae and Solanaceae was 97-130 Myr, the 95\% HPD for the age of the Convolvulaceae crown node was 94-129 Myr, the 95\% HPD for the age of the Solanaceae crown node was 67-99 Myr, and the 95\% HPD for the age of the Ipomoea crown node was 18-43 Myr. There was a 20.3-fold difference between the maximum inferred substitution rate (MPE) of $2.9 * 10^{-3}$ substitutions $\mathrm{Myr}^{-1}$ (on the Solanaceae stem branch), and the minimum inferred substitution rate of $1.4^{*} 10^{-4}$ substitutions $\mathrm{Myr}^{-1}$ (Fig. 2a and S1a). However, apart 
464

465

466

467

468

469

470

471

472

473

474

475

476

477

478

479

480

481

482

483

484

485

486

487

488

489

490

491

492

493

494

495

496

497

from the Solanaceae stem branch, inferred rates are within a range between $1.4^{*} 10^{-4}$ and $1.4 * 10^{-3}$ substitutions $\mathrm{Myr}^{-1}$, a 10-fold difference.

Calibration Strategy 2 resulted in younger divergence time estimates (Fig. 2b). The 95\% HPD for the age of the divergence between Convolvulaceae and Solanaceae was 89-129 Myr, the 95\% HPD for the age of the Convolvulaceae crown node was 86-126 Myr, the 95\% HPD for the age of the Solanaceae crown node was 64-94 Myr, and the 95\% HPD for the age of the Ipomoea crown node was 17-41 Myr. There was a 21.5-fold difference between the maximum inferred substitution rate of $3.2 * 10^{-3}$ substitutions $\mathrm{Myr}^{-1}$ (on the Solanaceae stem branch), and the minimum inferred substitution rate of $1.5^{*} 10^{-4}$ substitutions $\mathrm{Myr}^{-1}$ (Fig. $2 \mathrm{~b}$ and S1b). However, apart from the Solanaceae stem branch, inferred rates fall within a range between $1.5^{*} 10^{-4}$ and $1.5^{*} 10^{-3}$ substitutions $\mathrm{Myr}^{-1}$, a 10 -fold difference.

Calibration Strategy 3 resulted in the youngest divergence time estimates (Fig. 2). The 95\% HPD for the age of the divergence between Convolvulaceae and Solanaceae was 57-58 Myr, the 95\% HPD for the age of the Convolvulaceae crown node was 46-56 Myr, the 95\% HPD for the age of the Solanaceae crown node was 56-58 Myr, and the 95\% HPD for the age of the Ipomoea crown node was 10-21 Myr. There was a 59-fold difference between the maximum inferred substitution rate of $1.3 * 10^{-2}$ substitutions $\mathrm{Myr}^{-1}$ (on the Convolvulaceae stem branch), and the minimum inferred substitution rate of $2.1 * 10^{-4}$ substitutions $\mathrm{Myr}^{-1}$ (Fig. 2c and S1c). However, apart from the Convolvulaceae stem branch, inferred rates fall within a range between $1.5^{*} 10^{-4}$ and $3.5^{*} 10^{-3}$ substitutions $\mathrm{Myr}^{-1}$, a 16-fold difference.

Calibration Strategy 4 also resulted in younger divergence time estimates than Calibration Strategy 1 and 2 (Fig. 2). The 95\% HPD for the age of the divergence between Convolvulaceae and Solanaceae was 41-83 Myr, the 95\% HPD for the age of the Convolvulaceae crown node was 40-81 Myr, the 95\% HPD for the age of the Solanaceae crown node was 26-59 Myr, and the 95\% HPD for the age of the Ipomoea crown node was 8$25 \mathrm{Ma}$. There was a 17.9-fold difference between the maximum inferred substitution rate of $4.8^{*} 10^{-3}$ substitutions $\mathrm{Myr}^{-1}$ (on the Solanaceae stem branch), and the minimum inferred substitution rate of $2.7 * 10^{-4}$ substitutions $\mathrm{Myr}^{-1}$ (Fig. $2 \mathrm{~d}$ and S1d). However, apart from the Solanaceae stem branch, inferred rates fall within a range between $1.5^{*} 10^{-4}$ and $2.5^{*} 10^{-3}$ substitutions $\mathrm{Myr}^{-1}$, a 9.2-fold difference.

With a strict clock, Calibration Strategies 1-3 resulted in divergence time estimates that were very similar to each other, and to divergence time estimates with Calibration Strategy 1 and a UCLN relaxed clock (Fig. S2a-c; Fig. 2a). Inferred substitution rates were also very similar for Calibration Strategies 1-3 with a strict clock (Fig. S2a-c). With a strict 
clock, Calibration Strategy 4 resulted in markedly younger divergence time estimates than Calibration Strategies 1-3 with a strict clock and calibration Strategy 4 with a UCLN relaxed clock (Fig. S2d, Fig. 2d). For all four calibration strategies, 95\% HPDs were far narrower with a strict clock (Fig. 2, Fig. S2).

\subsection{Divergence time estimates within Ipomoea}

\subsubsection{Divergence time estimates within Ipomoea inferred in treePL with the optimum} smoothing value

When the optimum smoothing value of 0.01 was used to infer a nuclear timecalibrated phylogeny in treePL, the inferred crown ages for the New World and Old World Clades were approximately $29 \mathrm{Myr}$ and $28 \mathrm{Myr}$ respectively (Fig. 3a). The major clades A-E, and Astripomoea and close relatives, are inferred to have originated 16-23Ma, throughout the Early Miocene (Fig 3a). The Parana Clade and Coriaceous Sepal Clade, and the Batatas group are inferred to have originated 7.5-12.5 Ma, during the Late Miocene. In this timecalibrated phylogeny, there was a 3.279-fold difference between the minimum inferred substitution rate of $6.21 * 10^{-4}$ substitutions $\mathrm{Myr}^{-1}$, and the maximum inferred substitution rate of $2.036 * 10^{-3}$ substitutions Myr $^{-1}$ (Fig. 3a, Fig. S3a).

When the optimum smoothing value of 10000 was used to infer a chloroplast timecalibrated phylogeny in treePL, the inferred crown ages for the New World and Old World Clades were approximately 25.6 Myr and 21 Myr respectively (Fig. 3b). Clades A, B, D, E, and Astripomoea and close relatives, are inferred to have originated 13.5-19.1 Ma, throughout the Early and Middle Miocene. Clade $\mathrm{C}$ is inferred to have originated during the Late Miocene, 8.9 Ma (Fig. 3b). In this time-calibrated phylogeny, the same substitution rate of $1.81 * 10^{-4}$ substitutions $\mathrm{Myr}^{-1}$ was inferred for every branch (Fig. 3b).

These results from the nuclear and chloroplast time-calibrated phylogenies indicate that much of the morphological and species diversity within Ipomoea, briefly summarised in Figure $3 c-j$, originated before the end of the Miocene. However, the limited taxon sampling means that it is difficult to construct detailed or precise hypotheses about when and how frequently specific morphological traits evolved.

\subsubsection{Alternative divergence time estimates for the Ipomoea nuclear phylogeny}

Divergence times inferred in treePL with alternative smoothing values were similar to divergence times inferred with the optimum value, although in some cases they were somewhat younger (Fig. 4a, Fig. S4a-c). With higher smoothing values, the magnitude of among-branch-substitution-rate-variation decreased (Fig. S3a-c, Fig. S4a-c). 
For analyses in RevBayes, in the skeletal time-calibrated phylogeny inferred with a

532

533

534

535

536

537

538

539

540

541

542

543

544

545

546

547

548

549

550

551

552

553

554

555

556

557

558

559

560

561

562

563

564

strict clock, MPEs for the ages of major clades were similar to age estimates inferred in treePL (Fig. 4a, Fig. S5a). 95\% HPD intervals were on average 10.9\% of the MPE for the age each clade (Fig. 4a, Table S2), and the inferred substitution rate was similar to those inferred in treePL (Fig. S5a). In the three-taxon time-calibrated phylogenies inferred with a strict clock, MPEs for the ages of major clades were somewhat older than in the skeletal timecalibrated phylogeny and 95\% HPDs were on average 4.7\% of the MPE for the age of each clade (Fig. 4a, Table S2).

In the skeletal time-calibrated phylogenies inferred with a relaxed clock, MPEs for the ages of major clades were similar to age estimates inferred in treePL and to the skeletal timecalibrated phylogeny inferred with a strict clock. However, there was more variation in the relationship between these age estimates and other estimates, compared to those previously discussed, with inferred ages being either older or younger depending on the clade (Fig. 4a, Fig. S5b). 95\% HPD intervals were markedly wider than in RevBayes analyses with a strict clock, on average they were $60 \%$ of the MPE for the age of each clade (Fig. 4a, Table S2). The substitution rate and magnitude of among-branch-substitution-rate-variation was similar to that inferred in treePL with a lower smoothing value (Fig. S5b, Fig. S6a). In three-taxon time-calibrated phylogenies inferred with a relaxed clock, MPEs tended to be slightly older than MPEs inferred in either of the skeletal time-calibrated phylogenies. However, for the two oldest clades in the phylogeny, the New Word and Old World clades, MPEs were markedly younger than other age estimates inferred in treePL or RevBayes (Fig. 4a). 95\% HPD intervals were considerably wider compared to the skeletal time-calibrated phylogeny inferred with a relaxed clock. On average they were $114.1 \%$ of the MPE for the age of each clade (Fig. 4a, Table S2).

\subsubsection{Alternative divergence time estimates for the Ipomoea chloroplast phylogeny}

Divergence times inferred in treePL with a smoothing value of 100 were very similar to divergence times inferred with a smoothing value of 10000 (Fig. 4b, Fig. S4f). With a smoothing value of 0.01 or 1 , inferred divergence times were significantly older. This was especially the case for clades A-E, the majority of which were inferred to have originated during the earliest Miocene or Late Oligocene (Fig. 4b, Fig. S4d and e). With lower smoothing values, the magnitude of among-branch-substitution-rate-variation increased (Fig. S3d-f, Fig S4d-f). With the lowest smoothing value of 0.01, there was a 193-fold difference between the maximum inferred substitution rate of $3.86 * 10^{-4}$ substitutions $\mathrm{Myr}^{-1}$, and the minimum inferred substitution rate of $2 * 10^{-6}$ substitutions $\mathrm{Myr}^{-1}$. 

strict clock, MPEs for the ages of major clades were most similar to age estimates inferred in treePL with a smoothing value of 10000 (Fig. 4b, Fig. S5c). 95\% HPD intervals were approximately $16 \%$ of the MPE for the age of each clade (Fig. 4b, Table S2). As with

569

570 divergence times, the inferred substitution rate was also similar to when a smoothing value of 10000 was used in treePL (Fig. S5c). In three-taxon time calibrated phylogenies inferred with a strict clock, MPEs for the ages of major clades were similar to those inferred in the skeletal time-calibrated phylogeny (Fig. 4b). 95\% HPDs were slightly wider than in the skeletal timecalibrated phylogeny, approximately $19 \%$ of the MPE for each clade (Fig. 4b, Table S2).

In skeletal time-calibrated phylogenies inferred with a relaxed clock, MPEs for the ages of major clades tended to be slightly older than in either of the two types of timecalibrated phylogeny inferred with a strict clock (Fig. 4b, Fig. S5d). They were therefore also slightly closer to age estimates inferred in treePL with smoothing values of 0.01 and $1.95 \%$ HPDs were also markedly wider than RevBayes analyses with a strict clock, approximately $72 \%$ of the MPE for the age of each clade (Fig. 4b, Table S2). The substitution rate, and magnitude of among-branch-substitution-rate-variation was similar to the chloroplast timecalibrated phylogeny inferred in treePL with a smoothing value of 0.01 (Fig. S5d, Fig. S6b). In three-taxon time-calibrated phylogenies inferred with a relaxed clock, MPEs for the ages of major clades tended to be slightly older than MPEs inferred according to alternative methods in RevBayes (Fig. 4b). 95\% HPD intervals were also markedly wider, approximately $132.4 \%$ of the MPE for each clade (Fig. 4b, Table S2).

\subsection{Diversification parameter estimates within Ipomoea}

\subsubsection{Nuclear Phylogeny}

Inferred diversification parameters were similar, regardless of the smoothing value that was used to infer the time-calibrated phylogeny (Fig. 3a, Fig. S4a-c, Table 1). With the time-calibrated phylogeny inferred with the optimum smoothing value of 0.01 , a single shift in diversification parameters near the origin of a clade that contains the Parana Clade and Coriaceous Sepal Clade, has by far the highest posterior probability ( $p=0.9)$ (Fig. 3a). This diversification parameter shift leads to a 5.9-fold increase in the net diversification rate to 0.802 species $\mathrm{Myr}^{-1}$, compared to the background rate of 0.136 species $\mathrm{Myr}^{-1}$. This net diversification rate increase is caused by an increase in the inferred speciation rate from 0.147 , to 0.820 species $\mathrm{Myr}^{-1}$ (Table 1). The finding that there is a single shift in diversification parameters is supported by comparison of Bayes factors, the primary method of model comparison in a Bayesian framework. These comparisons provided strong support 
599

600

601

602

603

604

605

606

607

608

609

610

611

612

613

614

615

616

617

618

619

620

621

for a model with a single shift in diversification parameters, regardless of the smoothing value that was used to infer the time-calibrated phylogeny (Table S3).

\subsubsection{Chloroplast Phylogeny}

In the time-calibrated phylogeny inferred with the optimum smoothing value of 10000, a single shift in diversification parameters near the origin of the Parana Clade has the highest posterior probability ( $\mathrm{p}=0.79)$ (Fig. 3b). Two alternative patterns, in which there are shifts in diversification parameters on immediately ancestral branches, have significantly lower posterior probabilities (Fig. 3b). The most probable diversification parameter shift is inferred to lead to a 3.2-fold increase in net the diversification rate to 0.641 species $\mathrm{Myr}^{-1}$, compared to the background rate of 0.199 species $\mathrm{Myr}^{-1}$ (Table 1). This net diversification rate increase is caused by an increase in the speciation rate from 0.212 to 0.768 species $\mathrm{Myr}^{-1}$. With the time-calibrated phylogeny inferred with a smoothing value of 100 , inferred diversification parameters were very similar to when a smoothing value of 10000 was used (Fig. S4f, Table 1). With the time-calibrated phylogenies inferred with smoothing values of 1 and 0.01, no discrete shifts in diversification parameters were inferred (Fig. S5d and e, Table 1). These findings were supported by comparison of Bayes factors. For time-calibrated phylogenies inferred with smoothing values of 10000 and 100, there was strong support for a model with a single shift in diversification parameters. For time-calibrated phylogenies inferred with smoothing values of 1 and 0.01 , there was strong support for a model in which there were no discrete shifts in diversification parameters (Table S3).

Table 1. Diversification parameter estimates for Ipomoea inferred in BAMM using different time-calibrated phylogenies as input trees.

\begin{tabular}{|c|c|c|c|c|}
\hline Time-calibrated phylogeny & $\begin{array}{c}\text { Background } \\
\text { speciation rate } \\
\left(\text { species } \mathrm{Myr}^{-1}\right)\end{array}$ & $\begin{array}{c}\text { Background } \\
\text { extinction rate } \\
\left(\text { species } \mathrm{Myr}^{-1}\right)\end{array}$ & $\begin{array}{c}\text { Shift } \\
\text { speciation } \\
\text { rate* }^{*}(\text { species } \\
\left.\text { Myr }^{-1}\right)\end{array}$ & $\begin{array}{c}\text { Shift } \\
\text { extinction } \\
\text { rate* }^{*}(\text { species } \\
\left.\mathrm{Myr}^{-1}\right)\end{array}$ \\
\hline Nuclear, smoothing $=0.01$ & 0.147 & 0.0108 & 0.820 & 0.0176 \\
\hline Nuclear, smoothing $=1$ & 0.150 & 0.00942 & 0.878 & 0.0184 \\
\hline Nuclear, smoothing $=100$ & 0.150 & 0.00950 & 0.895 & 0.0182 \\
\hline Nuclear, smoothing $=10000$ & 0.150 & 0.00954 & 0.898 & 0.0190 \\
\hline Chloroplast, smoothing $=0.01$ & 0.153 & 0.0136 & --- & --- \\
\hline Chloroplast, smoothing $=1$ & 0.150 & 0.0123 & --- & --- \\
\hline Chloroplast, smoothing $=100$ & 0.212 & 0.0135 & 0.775 & 0.131 \\
\hline Chloroplast, smoothing $=10000$ & 0.212 & 0.0131 & 0.768 & 0.126 \\
\hline
\end{tabular}

* Shift speciation rate and Shift extinction rate refer to rates for clades within which there are significant shifts in diversification parameters compared to the background rate. The phylogenetic position of these rate shifts is indicated in Figure 2 and Figure S5.

625 


\subsubsection{When did the sweet potato diverge from its closest relative?}

In time-calibrated phylogenies for the Batatas group inferred in RevBayes with a UCLN relaxed clock, the divergence between I. batatas and I. trifida was inferred to have occurred at least 1.9Ma in the nuclear time-calibrated phylogeny (Fig. 5a), and at least 0.89Ma in the chloroplast time-calibrated phylogeny (Fig. 5b). In nuclear and chloroplast time-calibrated phylogenies inferred with alternative methodologies, the inferred divergence time between I. batatas and I. trifida was always inferred to have occurred at least $0.8 \mathrm{Ma}$ (Fig. S7, Fig. S8). These alternative methodologies make different assumptions about the magnitude and nature of among-branch-substitution-rate-variation. As such, each analysis makes different inferences about substitution rates on individual branches (Fig. 5, Fig. S7, Fig. S8). Regardless of this, in all cases the divergence between I. batatas and I. trifida is inferred to have occurred in pre-human times.

We performed two further analyses with our chloroplast dataset. Where we constrained the divergence between I. batatas and I. trifida to have occurred no more than 15 $\mathrm{Ka}$, but retained the point calibration of $6.2 \mathrm{Myr}$ at the root node - we inferred a greater than 200-fold increase in substitution rates for branches leading to I. batatas and I. trifida. As a result, substitution rates in this part of the time-calibrated phylogeny exceed $2.2 * 10^{-2}$ substitutions $\mathrm{Myr}^{-1}$ (Fig. 6a, Fig. S9). This value far exceeds any previously inferred substitution or mutation rate for chloroplast DNA. This result was consistent regardless of whether we used a UCLN relaxed clock (Fig. 6a) or a random local clock (Fig. S9).

Where we constrained the divergence between I. batatas and I. trifida to occur $15 \mathrm{Ka}$, but removed the point calibration at the root node, we inferred a substitution rate of $2.1 * 10^{-2}$ substitutions $\mathrm{Myr}^{-1}$ for the Batatas group (Fig. 6b). As before, this rate is far higher than any previously inferred substitution or mutation rate for chloroplast DNA. Taken together, these two additional analyses demonstrate that in order to infer a divergence time between $I$. batatas and I. trifida that occurred in human times, and that is consistent with the storage root of sweet potato having originated as a result of human domestication, a substitution rate would be required that far exceeds any known substitution or mutation rate for chloroplast DNA.

From our time-calibrated phylogenies for the entire of Ipomoea, we were also able to show that storage roots are likely to have evolved at least 30 times independently throughout Ipomoea, and that in each case the origin of the storage root occurred in pre-human times. This was the case regardless of the smoothing value used to infer the time-calibrated 

Ipomoea independently of human actions.

662

663

664

665

666

667

668

669

670

671

672

673

674

675

676

677

678

679

680

681

682

683

684

685

686

687

688

689

690

691

692

693

\subsubsection{When did diversity within the sweet potato evolve?}

With the time-calibrated phylogenies that contained all sampled accessions of $I$.

batatas and I. trifida, the divergences between most of the lineages within I. batatas occurred in pre-human times (Fig. 7). We interpret this as strong evidence that most of the diversity within I. batatas evolved in pre-human times. This result was consistent with both the nuclear (Fig. 7a) and chloroplast datasets (Fig. 7b), and for analyses inferred in treePL with both the optimum and alternative smoothing values, or in RevBayes with a strict clock or UCLN relaxed clock (Fig. S10, Fig. S11). These alternative methodologies make different assumptions about the magnitude and nature of among-branch-substitution-rate-variation. As such, each analysis makes different inferences about substitution rates on individual branches (Fig. 7, Fig. S10-12). Regardless of this, in all cases the divergences between different accessions of I. batatas and I. trifida are inferred to have occurred in pre-human times.

In RevBayes, we also inferred divergence times in three-taxon time-calibrated phylogenies. The divergence time estimates in these three-taxon time-calibrated phylogenies differed somewhat from those in which all taxa were sampled and HPDs were considerably wider. However, as before, the divergences between different accessions of I. batatas and $I$. trifida predominantly occurred in pre-human times (Table S5).

\section{DISCUSSION}

\subsection{Calibration uncertainty provides an uncertain basis for inferring the temporal} dynamics evolution in Ipomoea

Different combinations and interpretations of fossil calibrations provide an uncertain and conflicting framework for calibrating the phylogeny of Convolvulaceae and Solanaceae. As such, different calibration strategies led to major differences in inferred divergence times within the two families, including for the crown node of Ipomoea (Fig. 2). With respect to fossils within Solanaceae, this study focussed on the recently discovered Physalis infinemundi, and the implications of different assumptions about the maximum possible age of Solanaceae. We showed that making stronger assumptions that the Solanaceae stem node is significantly younger than $130 \mathrm{Myr}$ - a commonly used hard maximum for the origin of eudicots - led to significantly younger divergence time estimates. This was the case even when Physalis infinemundi was retained as a minimum constraint of 52Myr for the Solanoideae stem node. In Calibration Strategy 3, where we made the strongest assumption that the Solanaceae stem node was significantly younger than $130 \mathrm{Myr}$, age estimates were 
694

695

696

697

698

699

700

701

702

703

704

705

706

707

708

709

710

711

712

713

714

715

716

717

718

719

720

721

722

723

724

725

726

similar to those obtained in previous studies where significantly younger fossil calibrations were used (Särkinen et al. 2013; Magallón et al. 2015; Mitchell et al. 2016). As well as evaluating fossil calibrations within Solanaceae, we also constructed a time-calibrated phylogeny with a secondary calibration from Magallón et al. (2015). This led to younger divergence time estimates that were similar to Calibration Strategy 3.

It is impossible to make a definitive judgement about which calibration strategy is the most robust. Assuming its phylogenetic placement is correct, Physalis infinemundi does provide a minimum constraint for the Solanoideae stem node. However, it would be misleading to suggest we know a most probable age for the Solanoideae stem node in relation to the age of this fossil. Alternatively, it may be reasonable to suggest that the Solanaceae stem node must be younger than $130 \mathrm{Myr}$ - but the likely extent to which it is younger is also difficult to quantify. Further, the maximum constraint of $130 \mathrm{Ma}$ is derived from the observation that there is an absence of eudicot fossils in sediments older than this age, but a sudden appearance in younger sediments (Doyle et al. 1977). This maximum constraint is therefore based on the absence of evidence - a problematic basis upon which to construct assumptions.

The implications of different calibration strategies are also influenced by assumptions about among-branch-substitution-rate-variation. Note, for example, how the same calibration strategy can lead to significantly different divergence time estimates, depending on whether a UCLN relaxed clock or a strict clock is used (Fig. 2 and Fig. S2). Further, for the same molecular clock model, different calibration strategies lead to marked differences in inferred substitution rates (Fig. 2, Fig. S1, Fig. S2). Taken together, this highlights the tight interaction between fossil calibrations, assumptions about among-branch-substitution-ratevariation, inferred substitution rates, and inferred divergence times.

Despite the uncertainty presented here, we suggest that presenting our results in this way provides the most robust basis for interpreting divergence time estimates, and accounting for factors that may affect inferences in the future. We do not, by contrast, present a single time-calibrated phylogeny, because no single time-calibrated phylogeny fully accounts for all the uncertainty that is represented here. Despite this complexity, with careful interpretation we suggest these results can provide a basis for discussing the temporal dynamics of evolution in Ipomoea, and answering specific questions about the origin of I. batatas.

\subsection{Uncertainty about substitution rate variation within Ipomoea leads to further} uncertainty in divergence time estimates 
We did not implement any fossil calibrations at internal nodes within Ipomoea.

728 Therefore, whilst uncertainty about the absolute age of the entire of Ipomoea can result from

729

730

731

732

733

734

735

736

737

738

739

740

741

742

743

744

745

746

747

748

749

750

751

752

753

754

755

756

757

758

759

760 calibration uncertainty (summarised in Figure 2), uncertainty about the relative ages of different clades within Ipomoea cannot result from calibration uncertainty. Instead, it results predominantly from assumptions about substitution rates, and in some cases additional assumptions about relative clade ages (expressed by the birth-death branching process in Bayesian analyses). In our analyses of divergence times within Ipomoea, we therefore experimented with different assumptions about substitution rates, and in Bayesian analyses, with the assumptions of the birth-death branching process.

In nuclear time-calibrated phylogenies inferred in treePL, we showed that different smoothing values have a limited effect on inferred divergence times (Fig. 3a, Fig. 4a, Fig. S4a-c). Regardless of the smoothing value, the majority of major clades are inferred to have originated in the Late Oligocene and throughout the Early Miocene, and the Parana Clade, Coriaceous Sepal Clade, and the Batatas group are inferred to have originated in the Late Miocene. This implies that inferred divergence times within Ipomoea are fairly robust to assumptions about among-branch-substitution-rate-variation. This result is likely to be explained by the fact that regardless of the smoothing value, the magnitude of inferred among-branch-substitution-rate-variation was always fairly low (Fig. 3a, Fig. S3a-c, Fig. $\mathrm{S} 4 \mathrm{a}-\mathrm{c})$. Nonetheless, any conclusion about the robustness of these divergence time estimates assumes that the model of rate variation used in treePL - whereby substitution rates are correlated between ancestral and descendant branches (Sanderson 1997; Smith and O’Meara 2012) - is valid.

By contrast, in chloroplast time-calibrated phylogenies inferred in treePL, different smoothing values had a more profound effect on inferred divergence times. Specifically, lower smoothing values (0.01 and 1$)$ caused basal branches to be considerably shorter - and by consequence have considerably higher substitution rates - compared to higher smoothing values. This in turn meant that with lower smoothing values, inferred divergence times were considerably older such that the majority of major clades originated in the Late Oligocene or very early Miocene (Fig. 3b, Fig. 4b, Fig. S4d-f). Clearly, this result highlights that there is considerable uncertainty in divergence time estimates within Ipomoea. However, when experimenting with different smoothing values, we performed extensive cross-validation analyses to determine which smoothing values are most appropriate. For the chloroplast data, these suggested that higher smoothing values are more appropriate. Further, we suggest that divergence times inferred with higher smoothing values are more congruent with the nuclear 
time-calibrated phylogeny, with major clades originating throughout the Early Miocene, rather than exclusively in the very early Miocene. We therefore tentatively suggest that divergence times inferred with higher smoothing values are more accurate (Fig. 3b).

For analyses performed with nuclear data in RevBayes, the skeletal and three-taxon time-calibrated phylogenies were broadly congruent with those inferred in treePL (Fig. 4a, Fig. S5a and b). This provides further evidence that divergence time estimates with this dataset are relatively insensitive to assumptions about among-branch-substitution-ratevariation. However, we note that 95\% HPDs are considerably wider when a UCLN relaxed clock is used compared to a strict clock (Fig. 4a, Table S2). This highlights how uncertainty about substitution rates for individual branches - a source of uncertainty that is explicitly accounted for with a UCLN relaxed clock but not with a strict clock - leads to uncertainty in divergence time estimates. We also note that when a UCLN relaxed clock is used, 95\% HPD widths are considerably wider in three-taxon time calibrated phylogenies compared to skeletal time-calibrated phylogenies (Fig. 4a, Table S2). We suggest this reflects the fact that in the skeletal time-calibrated phylogenies, the birth-death branching process - which makes assumptions about divergence times - causes greater precision in divergence time estimates. This contrasts to the three-taxon time-calibrated phylogenies, where the birth-death branching process does make any assumptions about divergence times. This result has concerning implications because it suggests that the precision of divergence time estimates is sensitive to the assumption of the birth-death branching process that diversification parameters are constant. Given that this assumption is often likely to be violated, this precision may be misleading. Nonetheless, we tentatively suggest that the uncertainty expressed in the threetaxon time-calibrated phylogenies may be excessive given that all the time-calibrated phylogenies inferred in treePL corroborate with each other, and with the skeletal timecalibrated phylogenies inferred in RevBayes. However, we make this assertion whilst also considering the potential sources of error outlined above.

For analyses performed with chloroplast data in RevBayes, the skeletal and threetaxon time-calibrated phylogenies were broadly congruent with those inferred in treePL with smoothing values of 100 and 10000 (Fig. 4b, Fig. S5c and d). Given that smoothing values of 100 and 10000 are likely to be more appropriate for this dataset, and corroborate inferences derived from nuclear data, this result supports our previous inferences about when major clades within Ipomoea evolved (Fig. 3). Like with the nuclear data, 95\% HPDs were considerably wider when a UCLN relaxed clock was used, and when divergence times were 
inferred in three-taxon time-calibrated phylogenies (Fig. 4b, Table S2). We suggest the same explanations as in the discussion of nuclear time-calibrated phylogenies.

Taken together, these results provide tentative support that the majority of major clades within Ipomoea evolved in the Late Oligocene and throughout the Early Miocene, and the Parana Clade, Coriaceous Sepal Clade, and the Batatas group evolved in the Late Miocene. However, these conclusions are dependent on several important variables. These include the Calibration Strategy used to infer the time-calibrated phylogeny for Convolvulaceae and Solanaceae, the assumptions of different methods that account for among-branch-substitution-rate-variation, and - in Bayesian analyses - the assumption of a constant rate of speciation and extinction. It is important to consider the effect of these variables when interpreting the divergence time estimates presented here. Specifically, the calibration strategy will affect inferences of the absolute age of the entire of Ipomoea, whilst assumptions about the magnitude and nature of among-branch-substitution-rate-variation and the constancy of speciation and extinction rates, will affect inferences of the relative ages of different clades.

\subsection{Significant diversification parameter variation within Ipomoea is highly likely}

Inferences of diversification parameters are sensitive to uncertainty and error in divergence time estimates. In order to account for this, we inferred diversification parameters on time-calibrated phylogenies inferred with different smoothing values in treePL. With the nuclear time-calibrated phylogenies, we inferred a significant net diversification rate increase for the clade that contains both the Parana Clade and Coriaceous Sepal Clade. This net diversification rate increase was strongly supported, regardless of the smoothing value (Fig. 3a, Fig. S4a-c, Table 1). We were not surprised to infer a net diversification rate increase for this clade. Species within the Parana Clade and Coriaceous Sepal Clade often have very localised geographical ranges, contain groups of species whose morphologies overlap significantly, but overall, exhibit a variety of different growth forms and occur in a range of different habitats. These are characteristic attributes of rapid evolutionary radiations (Hughes and Eastwood. 2006; Givnish et al. 2009; Nevado et al. 2016).

With the chloroplast time-calibrated phylogenies inferred with smoothing values of 100 and 10000, we inferred a significant net diversification rate increase in the Parana Clade but not the Coriaceous Sepal Clade. By contrast, we did not infer any significant shifts in diversification parameters when a smoothing value of 0.01 or 1 was used (Fig. 3b, Fig. S4d-f, Table 1). However, given our cross-validation analyses suggest smoothing values of 100 and 10000 are more appropriate, we suggest the chloroplast dataset supports a significant net 
diversification rate increase at the origin of the Parana Clade. Taken together, the nuclear and chloroplast time-calibrated phylogenies provide strong support for a significant net diversification rate increase at either the origin of the clade containing the Parana Clade and Coriaceous Sepal Clade, or at the origin of the Parana Clade.

Despite the likely presence of net diversification rate shifts within Ipomoea, there is considerable uncertainty about the nature of these shifts. First, the possibility of erroneous divergence time estimates means there is considerable uncertainty about when the net diversification rate shifts actually occurred. Although we accounted for divergence time estimation error to an extent, by inferring diversification parameters on time-calibrated phylogenies inferred with different smoothing values, we did not account for other factors. For example, calibration uncertainty may mean that the entire phylogeny is calibrated to an incorrect timescale. A further factor that will likely affect the inferred timing and number of net diversification rate shifts, is the limited sampling within the phylogeny. For example, in the Parana Clade, only around 33\% of the species are sampled. Although we corrected for this in BAMM by specifying sampling proportions, a robust and well-resolved phylogeny that includes these missing species may result in the inference of several nested net diversification rate shifts that could not be recovered in the analyses presented here.

A further aspect of these net diversification rate shifts for which considerable uncertainty remains, is whether they reflect changes in the speciation rate or extinction rate. Our analyses in BAMM suggest they result from significant increases in the speciation rate (Table 1). However, in many cases it is likely to be very difficult to determine the relative contribution of speciation rate changes and extinction rate changes on inferred diversification dynamics (Rabosky 2010, 2014; Sanderson and Donoghue 2015; Moore et al. 2016). This is primarily because extinct branches are not sampled in a molecular phylogeny. Uncertainty about the relative importance of changes in speciation rates and changes in extinction rates will have profound implications for how hypothesis are constructed to explain the net diversification rate shifts discussed here. Further, a failure to sample extinct branches may mean that other aspects of the diversification history of Ipomoea have been entirely overlooked in this study.

\subsection{The sweet potato evolved in pre-human times}

We inferred time-calibrated phylogenies for Ipomoea in treePL using a point calibration for the root node that was equal to the MPE for the age of Ipomoea from Calibration Strategy 4. We suggest this calibration leads to significant underestimation of divergence times within Ipomoea, because the calibration strategy from which it is derived is 
862

863

864

865

866

867

868

869

870

871

872

873

874

875

876

877

878

879

880

881

882

883

884

885

886

887

888

889

890

891

892

893

894

895

likely to bias estimates to younger ages. Biasing our analysis in this way represents the most robust test of whether the storage root of I. batatas evolved in pre-human times. In these time-calibrated phylogenies, the divergence between $I$. batatas and its closest wild relative $I$. trifida was always in pre-human times. This was the case regardless of the smoothing value, or whether the phylogeny was inferred from nuclear or chloroplast data (Fig. S7a-d, Fig. S8ad). When we also inferred divergence times between $I$. batatas and its closest wild relatives in a Bayesian framework, the inferred divergence between I. batatas and I. trifida also significantly predated human times. This was the case regardless of whether a strict clock or a UCLN relaxed clock was used, or whether the phylogeny was inferred with nuclear or chloroplast data (Fig. 5, Fig. S7f, Fig. S8f). I. batatas is the only species amongst its close relatives that possesses a storage root, and together these results indicate that the storage root of I. batatas evolved in pre-human times.

We queried the conclusion that the divergence between I. batatas and I. trifida occurred in pre-human times by performing analyses with the chloroplast dataset where the divergence between I. batatas and I. trifida was constrained such that it occurred $15 \mathrm{Ka}$. In order to satisfy this calibration, we showed that the substitution rate for the chloroplast would be $2 * 10^{-2}$ substitutions $\mathrm{Myr}^{-1}$ (Fig. 6, Fig. S9). This is far higher than any previously recorded substitution or mutation rate for chloroplast DNA. Although there might be an expectation of elevated substitution rates for some genes in association with domestication, we would not expect such large rate differences to be observed across the entire plastome. Further, the 15 Ka constraint that we used is likely to represent an upper limit of when humans began domesticating crops, with most estimates for the origin of crop domestication being closer to $10 \mathrm{Ka}$ (Feldman 2001; Hillman et al. 2001; Zohary et al. 2006; Kingsbury 2009). To satisfy this younger timescale, such that the divergence between I. batatas and I trifida was constrained to have occurred $10 \mathrm{Ka}$, even higher substitution rates would be required than are recorded here.

The conclusion that the storage root of I. batatas evolved in pre-human times is consistent with the observation that storage roots are likely to have evolved repeatedly throughout Ipomoea, and each origin of the storage root in Ipomoea occurred in pre-human times (Table S4; Munoz et al. 2019). This indicates the capacity for storage roots to evolve in Ipomoea independently of human action.

We also showed that the majority of diversity within I. batatas is likely to have evolved in pre-human times. In our time-calibrated phylogenies constructed from all sampled accessions of $I$. batatas and I. trifida, divergence events between different accessions 
896

897

898

899

900

901

902

903

904

905

906

907

908

909

910

911

912

913

914

915

916

917

918

919

920

921

922

923

924

925

926

927

928

significantly predated human times (Fig. 7, Fig. S10-11, Table S5). This was the case regardless of the method used. These different methods included using treePL with different smoothing values, Bayesian analyses with a strict clock and UCLN relaxed clock, and Bayesian analyses with three-taxon time-calibrated phylogenies that removed temporal assumptions associated with the birth-death tree prior.

\subsection{Assimilating uncertainty and making conclusions}

It is challenging to assimilate the uncertainty in the results presented here. By highlighting the assumptions that underpin different methods, the different possibilities of potential uncertainty can appear immense. In most studies performed in a Bayesian framework, the 95\% HPD is presented as an effective way of accounting for uncertainty. However, as demonstrated here, the 95\% HPD is entirely dependent on the assumptions that are made when performing an analysis. When the validity of different sets of assumptions is difficult to distinguish, as is often the case when inferring divergence times and diversification rates, no single 95\% HPD is therefore capable of reflecting the uncertainty inherent in these different assumptions. Thus, a more valuable approach for future studies, as followed here, may be to comprehensively account for the different types of assumptions that underlie inferences about the temporal dynamics of evolutionary diversification and openly discuss the implications of these. This may not provide simple answers to questions, but may provide a more robust foundation upon which future studies of macro-evolution can rest.

Despite the uncertainty we present here, we stress that when questions are carefully framed, and results carefully interpreted, novel and robust inferences about the temporal dynamics of evolutionary history can be made. In the analyses we present here, this includes the inference that the storage root of I. batatas evolved in pre-human times and was not a result of human domestication, and the inference that there is likely to be at least one significant shift in diversification rates within Ipomoea.

Regardless, in some cases it remains difficult to perform analyses from which robust inferences and confident conclusions about the temporal dynamics of evolutionary history can be made. Even when this is the case, for example with respect to the age of Ipomoea or major clades within Ipomoea, or the timing and frequency of diversification rate shifts, exploring the uncertainty in these inferences is invaluable. It can provide a basis upon which to develop new hypotheses that can be meaningfully tested with available data in the future, as well as providing a framework for prioritising the collection of new data. Further, it can stimulate theoretical and methodological studies that aim to characterise the extent to which 
929

930

931

932

933

934

935

936

937

938

939

940

941

942

943

944

945

946

947

948

949

950

951

952

953

954

955

956

957

958

959

960

961

962

963

964

965

966

967

968

969

970

971

972

inferences about macroevolutionary history can be made from analysing molecular phylogenies.

\section{FUNDING}

This work was supported by a NERC scholarship granted through the Environmental

Research DTP programme to TC.

\section{REFERENCES}

Baldwin BG and Sanderson MJ. 1998. Age and rate of diversification of the Hawaiian silversword alliance (Compositae). Proc Natl Acad Sci USA. 95:9402-9406.

Britton T. 2005. Estimating Divergence Times in Phylogenetic Trees Without a Molecular Clock. Syst. Biol. 54:500-507.

Bromham L. 2006. Molecular dates for the Cambrian Explosion: is the light at the end of the tunnel an oncoming train? Palaeontol Electron. 9:2004-2006.

Cardillo M, Weston PH, Reynolds ZKM, Olde PM, Mast AR, Lemmon EM, Lemmon AR, Bromham L. 2017. The phylogeny and biogeography of Hakea (Proteaceae) reveals the role of biome shifts in a continental plant radiation. Evolution. 71:1928-1943.

Castresana J. 2000. Selection of conserved blocks from multiple alignments for their use in phylogenetic analysis. Mol Biol Evol. 17:540-552.

Crisp MD, Arroyo MTK, Cook LG, Gandolfo MA, Jordan GJ, McGlone MS, Weston PH, Westoby M, Wilf P, Linder HP. 2009. Phylogenetic biome conservatism on a global scale. Nature. 458:754756.

Dexter KG, Lavin M, Torke BM, Twyford AD, Kursar TA, Coley PD, Drake C, Hollands R, Pennington RT. 2017. Dispersal assembly of rain forest tree communities across the Amazon basin. Proc. Natl. Acad. Sci. USA. 114:2645-2650.

Donoghue MJ. 2008. A phylogenetic perspective on the distribution of plant diversity. Proc Natl Acad Sci USA. 105:11549-11555.

Donoghue MJ and Sanderson MJ. 2015. Confluence, synnovation, and depauperons in plant diversification. New Phytol. 207:260-274.

Doyle J. A., Biens P., Dorenkamp A., Jardiné S. 1977. Angiosperm pollen from the pre-Albian Cretaceous of Equatorial Africa. Bull. Cent. Rech. Explor. 1:451-473.

Drummond AJ, Ho SYW, Phillips MJ, Rambaut A. 2006. Relaxed Phylogenetics and Dating with Confidence. PLOS Biol. 4:e88.

Drummond AJ and Suchard MA. 2010. Bayesian random local clocks, or one rate to rule them all. BMC Biol. 8:114.

Feldman M. 2001. Origin of Cultivated Wheat. pp. 3-58. in Bonjean AP. and Angus WJ. eds. The World Wheat Book: A History of Wheat Breeding. Lavoisier: Paris.

Fitch WM. 1976. Molecular evolutionary clocks. pp. 160-178 in Ayala FJ eds. Molecular Evolution. Sinauer: Sunderland. 
Folk RA, Stubbs RL, Mort ME, Cellinese N, Allen J, Soltis PS, Soltis DE, Guralnick RP. 2019. Rates of niche and phenotype evolution lag behind diversification in a temperate radiation. Proc Natl Acad Sci USA. 116: 10874-10882

977

Gillespie J. H. 1991. The Causes of Molecular Evolution. Oxford: Oxford University Press.

979 Givnish TJ, MIllam KC, Mast AR, Paterson TB, Theim TJ, Hipp AL, Henss JM, Smith JF, Wood

980 KR, Sytsma KJ. 2009. Origin, adaptive radiation and diversification of the Hawaiian lobeliads

981 (Asterales: Campanulaceae). Proc. R. Soc. B. 276:407-416.

982 Govindarajulu R, Hughes CE, Bailey CD. 2011. Phylogenetic and population genetic analyses of diploid Leucaena (Leguminosae; Mimosoideae) reveal cryptic species diversity and patterns of divergent allopatric speciation. Am. J. Bot. 98:2049-2063.

Hillman G, Hedges R, Moore A, Colledge S, Pettitt P. 2001. New evidence of Lateglacial ceresl cultivation in Abu Hureyra on the Euphrates. Holocene. 11:383-393.

987 Hohna S, Heath TH, Boussau B, Landis MJ, Ronquist F, Huelsenbeck JP. 2014. Probabilistic

988 Graphical Model Representation in Phylogenetics. Syst Biol. 63:753-771.

989 Hohna S, Landis MJ, Heath TA, Boussau B, Lartillot N, Moore BR, Huelsenbeck JP, Ronquist F.

2016. RevBayes: Bayesian Phylogenetic Inference Using Graphical Models and an Interactive ModelSpecification Language. Syst Biol. 65:726-736.

992 Huelsenbeck JP and Ronquist F. 2001. MRBAYES: Bayesian inference of phylogenetic trees.

993 Bioinformatics. 17:754-755.

994 Hughes E. and Eastwood R. 2006. Island radiation on a continental scale: Exceptional rates of plant 995 diversification after uplift of the Andes. Proc Natl Acad Sci USA. 103:10334-10339.

996 Hughes CE, Pennington RT, Antonelli A. 2013. Neotropical Plant Evolution: Assembling the Big 997 Picture. Bot. J. Linn. Soc. 171:1-18.

998 Katoh K. 2002. MAFFT: a novel method for rapid multiple sequence alignment based on fast Fourier 999 transform. Nucleic Acids Res. 30:3059-3066.

1000 Katoh K and Standley DM. 2013. MAFFT Multiple Sequence Alignment Software Version 7: 1001 Improvements in Performance and Usability. Mol Biol Evol. 30:772-780.

1002 Kingsbury N. 2009. Hybrid. The University of Chicago Press: Chicago.

1003 Koenan EJM, de Vos JM, Aitchison GW, Simon MF, Schrire BD, de Sousa ER, de Queiroz LP, 1004 Hughes CE. 2013. Exploring the tempo of species diversification in legumes. S. Afr. J. Bot. 89:19-30.

Lavin M and Pennington RT. 2015. The contrasting nature of woody plant species in different

1007 Lavin M, Schrire BP, Lewis G, Pennington RT, Delgado-Salinas A, Thulin M, Hughes CE, Matos AB, Wojciechowski MF. 2004. Metacommunity process rather than continental tectonic history better explains geographically structured phylogenies in legumes. Philos. Trans. Royal Soc. 359:1509-1522.

1010 Magallón S, Gómez-Acevedo S, Sánchez-Reyes LL, Hernández-Hernández T. 2015. A Phytol. 207:437-453.

Magallón S, Hilu KW, Quandt D. 2013. Land plant evolutionary timeline: Gene effects are secondary to fossil constraints in relaxed clock estimation of age and substitution rates. Am J Bot. 100:556-573. 
Mitchell TC, Williams BRM, Wood JRI, Harris DJ, Scotland RW, Carine MA. 2016. How the temperate world was colonised by bindweeds: biogeography of the Convolvuleae (Convolvulaceae). BMC Evol. Biol. 16:16.

Moore BR, Höhna S, May MR, Rannala B, Huelsenbeck JP. 2016. Critically evaluating the theory and performance of Bayesian analysis of macroevolutionary mixtures. Proc. Natl. Acad. Sci. USA. 113:9569-9574.

Morris JL, Puttick MN, Clark JW, Edwards D, Kenrick P, Pressel S, Wellman CH, Yang Z, Schneider H, Donoghue PCJ. 2018. The timescale of early land plant evolution. Proc Natl Acad Sci USA. 115:E2274-E2283.

Muñoz-Rodríguez P, Carruthers T, Wood JRI, Williams BRM, Weitemier K, Kronmiller B, Ellis D, Anglin NL, Longway L, Harris SA, Rausher MD, Kelly S, Liston A, Scotland RW. 2018. Reconciling Conflicting Phylogenies in the Origin of Sweet Potato and Dispersal to Polynesia. 28:1246-1256.

Muñoz-Rodríguez P, Carruthers T, Wood JRI, Williams BRM, Weitemier K, Kronmiller B, Goodwin Z, Sumadijaya A, Anglin NL, Filer D, Harris D, Rausher MD, Kelly S, Liston A, Scotland RW. 2019. Assessing the biodiversity of morning glories at different scales using global natural history collections. Nat Plants, in press.

Nevado B, Atchison GW, Hughes CE, Filatov DA. 2016. Widespread adaptive evolution during repeated evolutionary radiations in New World lupins. Nat. Commun. 7:1-9.

Pennington RT, Lavin M, Oliveira-Filho A. 2009. Woody Plant Diversity, Evolution, and Ecology in the Tropics: Perspectives from Seasonally Dry Tropical Forests. 40:437-457. Annu. Rev. Ecol. Evol. Syst. 40:437-457.

Rabosky DL. 2014. Automatic detection of key innovations, rate shifts, and diversity-dependence on phylogenetic trees. PLOS ONE 9:e89543.

Rabosky DL, Chang J, Title OP, Cowmann PF, Sallan L, Friedman M, Kaschner K, Garilao C, Near TJ, Coll M, Alfaro ME. 2018. An inverse latitudinal gradient in speciation rate for marine fishes. Nature. 559:392-395.

Rabosky DL, Lovette IJ. 2008. Explosive Evolutionary Radiations: Decreasing Speciation or Increasing Extinction Through Time? Evolution. 62:1866-1875.

Rambaut A, Suchard MA, Xie D, Drummond AJ. 2014. Tracer v1.6 Available at: http://beast.bio.ed.ac.uk/Tracer

dos Reis M. and Yang Z. 2013. The unbearable uncertainty of Bayesian divergence time estimation. $J$. Syst. Evol. 51:30-43.

Ronquist F, Teslenko M, Van Der Mark P, Ayres DL, Darling A, Höhna S, Larget B, Liang L, Suchard MA, Huelsenbeck JP. 2012. MrBayes 3.2: Efficient Bayesian Phylogenetic Inference and Model Choice Across a Large Model Space. Syst Biol. 61:539-542.

Sanderson MJ. 1997. A Nonparametric Approach to Estimating Divergence Times in the Absence of Rate Constancy. Mol Biol Evol. 14:1218-1231.

Sanderson MJ and Doyle JA. 2001. Sources of error and confidence intervals in estimating the age of angiosperms from $r b c L$ and 18S rDNA data. Am. J. Bot. 88:1499-1516.

Särkinen T, Pennington RT, Lavin M, Simon MF, Hughes CE. 2012. Evolutionary islands in the Andes: persistence and isolation explain high endemism in Andean dry tropical forests. J Biogeogr. 39:884-900. 
Särkinen T, Bohs L, Olmstead RG, Knapp S. 2013. A phylogenetic framework for evoluitonary study of the nightshades (Solanaceae): a dated 1000 tip tree. BMC Evol Biol. 13:214.

1067 Simon MF, Grether R, de Queiroz LP, Skema C, Pennington RT, Hughes CE. 2009. Recent assembly 1068 of the Cerrado, a neotropical plant diversity hotspot, by in situ evolution of adaptations to fire. Proc.

1069 Natl. Acad. Sci. USA. 106:20359-20364.

1070 Simoes AR, Culham A, Carine M. 2015. Resolving the unresolved tribe: a molecular phylogenetic framework for the Merremieae (Convolvulaceae). Bot. J. Linn. Soc. 179:374-387.

1072 Smith SA and O'Meara BC. 2012. treePL: divergence time estimation using penalized likelihood for 1073 large phylogenies. Bioinformatics. 28:2689-2690.

1074 Srivastava G, Mehrotra RC, Dilcher DL. 2018. Paleocene Ipomoea (Convolvulaceae) from India with 1075 implications for an East Gondwana origin of Convolvulaceae. Proc Natl Acad Sci USA. 115:602810766033.

Stefanović S, Austin DF, Olmstead RG. 2003. Classification of Convolvulaceae: A Phylogenetic Approach. Syst. Biol. 28:791-806.

1080 Talavera G and Castresana J. 2007. Improvement of Phylogenies after Removing Divergent and 1081 Ambiguously Aligned Blocks from Protein Sequence Alignments. Syst Biol. 56:564-577.

1082 Thorne JL, Kishino H, Painter IS. 1998. Estimating the rate of evolution of the rate of molecular 1083 evolution. Mol Biol Evol. 15:1647-1657.

1092 Wilf P. and Escapa IH. 2015. Green Web or megabiased clock? Plant fossils from Gondwanan

Vaidya G, Lohman DJ, Meier R. 2011. SequenceMatrix: concatenation software for the fast assembly of multi-gene datasets with character set and codon information. Cladistics. 27:171-180.

Warnock RCM, Parham JF, Joyce WG, Tyler R, Donoghue PCJ. 2015. Calibration uncertainty in molecular dating analyses: there is no substitute for the prior evaluation of time priors. Proc $R$ Sol B. 282:20141013.

Wilf P, Carvalho MR, Gandalfo MA, Ruben Cuneo N. 2017. Eocene lantern fruits from Gondwanan Patagonia and the early origins of Solanaceae. Science. 355:71-75.

1094 Zohary D, Hopf M, Weiss E. 2006. Domestication of Plants in the Old World. Oxford University 1095 Press: Oxford.

1096 Zuckerkandl E. and Pauling LB. 1962. Molecular disease, evolution, and genetic heterogeneity. pp. 1097 189-225. in Kasha M. and Pullman B. eds. Horizons in biochemistry. Academic Press: New York.

1098 Zuckerkandl E. and Pauling L. 1965. Evolutionary Divergence and Convergence. pp. 97-166 in 1099 Bryson V. and Vogel HJ. eds. Evolving genes and proteins. Academic Press: New York.

Zhu T., dos Reis M., Yang Z. 2015. Characterization of the Uncertainty of Divergence Time Estimation under Relaxed Molecular Clock Models Using Multiple Loci. Syst. Biol. 64:267-280.

\section{FIGURE CAPTIONS}

Figure 1. A summary of analyses performed in this study. The dark grey boxes in the centre of the figure illustrate the progression from a sequence alignment, to a phylogeny, to a timecalibrated phylogeny, to inferring diversification parameters. The light grey boxes summarise the assumptions and issues associated with each step, and the way in which they were accounted for in this study. 
1112 Figure 2. Time-calibrated phylogenies for Convolvulaceae and Solanaceae inferred with different calibration strategies and a UCLN relaxed clock. Branch colours refer to the inferred substitution rate (MPE). Black circles refer to calibrated nodes. The grey " 1 " refers to the Solanaceae stem node (divergence between Solanaceae and Convolvulaceae), the grey " 2 " refers to the Convolvulaceae crown node, the grey " 3 " refers to the Solanaceae crown node, the grey " 4 " refers to the Ipomoea crown node. Grey bars refer to 95\% HPD intervals for the ages of these nodes. The time axis includes the geological period: $\mathrm{K}=$ Cretaceous, $\mathrm{P}=$ Paleogene, $\mathrm{Ng}=$ Neogene, $\mathrm{Q}=$ Quaternary. a) Calibration Strategy 1 - the Solanaceae stem node has a maximum age constraint of 130Myr, and the Solanoideae stem node has a minimum age constraint of 52Myr. b) Calibration Strategy 2 - the Solanaceae stem node is calibrated with a lognormal distribution with offset $=52, m=10.4, v=10.4$. The Solanoideae stem node has a minimum age constraint of 52Myr. c) Calibration Strategy $3-$ the Solanaceae stem node is calibrated with a lognormal distribution with offset $=52, m=5.2, v$ $=2.1$. The Solanoideae stem node has a minimum age constraint of 52Myr. d) The Solanaceae stem node is calibrated with a secondary calibration from Magallon et al. (2015). This is implemented as a normal distribution with $\mu=67.34, \sigma=9.980$.

Figure 3. Time-calibrated phylogenies for Ipomoea inferred in treePL with the optimum smoothing value, and images of species from different clades. A point calibration of 30.3 Myr is used at the root node in both a) and b). This is the MPE for the age of Ipomoea from Calibration Strategy 1. Where rates differ between branches, branch colours refer to the inferred substitution rate. Labelled nodes refer to nodes defining major clades. A - E refer to clades A - E from Muñoz-Rodríguez et al. (2019), IA refers to Astripmoea and close relatives, NW and OW refer to the New World and Old World clades (see Muñoz-Rodríguez et al. 2019), par refers to the Parana Clade, cs refers to the Coriaceous Sepal Clade, and bat refers to the Batatas group. Labelled arrows refer to the positions of shifts in diversification parameters inferred in BAMM. The number next to each arrow refers to the posterior probability of the shift occurring at that position in the phylogeny. Only shifts with a posterior probability of $>0.05$ are indicated. The time axis includes the geological epoch: $\mathrm{Ol}$ = Oligocene, $\mathrm{M}=$ Miocene, $\mathrm{P}=$ Pliocene, $\mathrm{Ps}=$ Pleistocene, and $\mathrm{H}=$ Holocene . a) shows a nuclear time-calibrated phylogeny, and b) shows a chloroplast time-calibrated phylogeny. $\mathbf{c}$ ) I. cordatotriloba, a trailing and twining species in the Batatas group. d) I. nil, a twining species in clade B. e) I. cairica, a trailing and twining species in clade E. f) I. bonariensis, a trailing and twining species in the Coriaceous Sepal Clade. g) I. paraguariensis, an erect species in the Parana clade. h) I. paludosa, an erect species in the Parana clade. i. $I$. psammophila, a trailing species in the Parana clade. j) I. megapotamica, a trailing and climbing species in the Parana clade.

Figure 4. Inferred ages for major clades within Ipomoea in nuclear a) and chloroplast b) time-calibrated phylogenies. Major clades and geological periods are labelled as set out in Figure 3. Different coloured points refer to age estimates derived from different inference methods (see key). For treePL these age estimates refer to point age estimates, for RevBayes these age estimates refer to MPEs. Where RevBayes has been used, the grey bar refers to the 95\% HPD.

Figure 5. Time-calibrated phylogenies for the Batatas group inferred in RevBayes with a UCLN relaxed clock. Branch colours refer to the inferred substitution rate. The grey bar refers to the 95\% HPD for the divergence between I. batatas and I. trifida. a) shows the nuclear time-calibrated phylogeny, b) shows the chloroplast time-calibrated phylogeny. 
1162 Figure 6. Alternative chloroplast time-calibrated phylogenies for the Batatas group inferred in RevBayes. Where rates differ between branches, branch colours refer to the inferred substitution rate. In a) the divergence between I. batatas and I. trifida is calibrated to $15 \mathrm{Kyr}$ and the root node has a point calibration of $6.18 \mathrm{Myr}$. This is the same root calibration as was used in all the previous time-calibrated phylogenies for the Batatas group inferred in RevBayes. A UCLN relaxed clock is used to infer substitution rates. In b) the divergence between I. batatas and I. trifida is constrained to have occurred $15 \mathrm{Ka}$ and there is no calibration at the root node. A strict clock is used to infer substitution rates.

Figure 7. Time-calibrated phylogenies for I. batatas and I. trifida inferred in treePL with the optimum smoothing value. All sampled accessions of the two species are included. Branch colours refer to the inferred substitution rate. a) shows the nuclear time-calibrated phylogeny, b) shows the chloroplast time-calibrated phylogeny. 
a)

Calibration Strategy 1

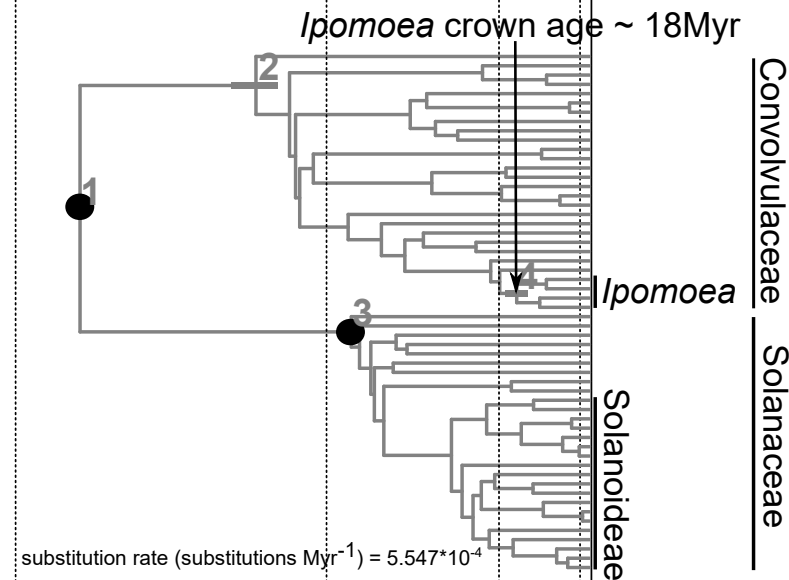

b)

Calibration Strategy 2

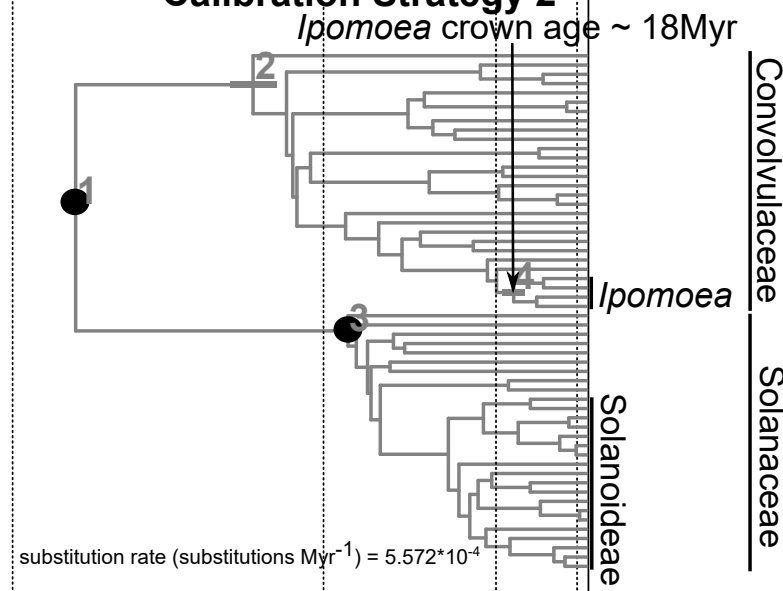

c)

Calibration Strategy 3

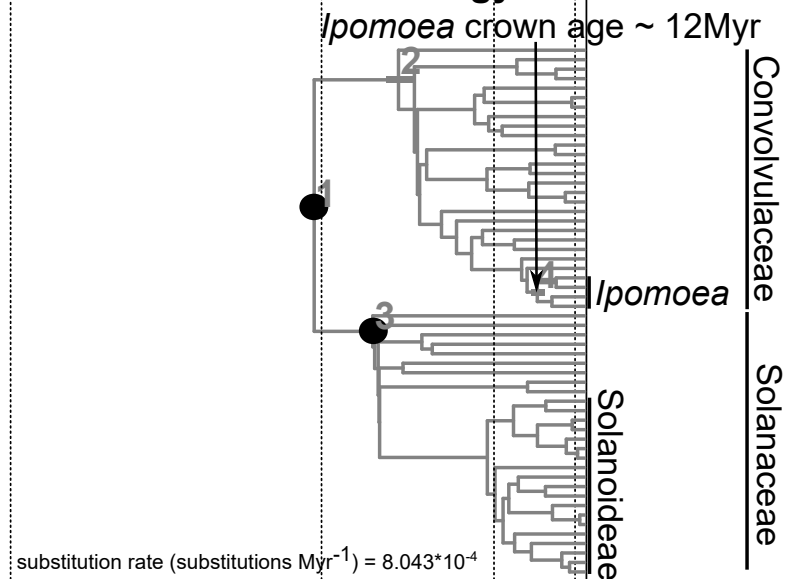

d) Calibration Strategy 4

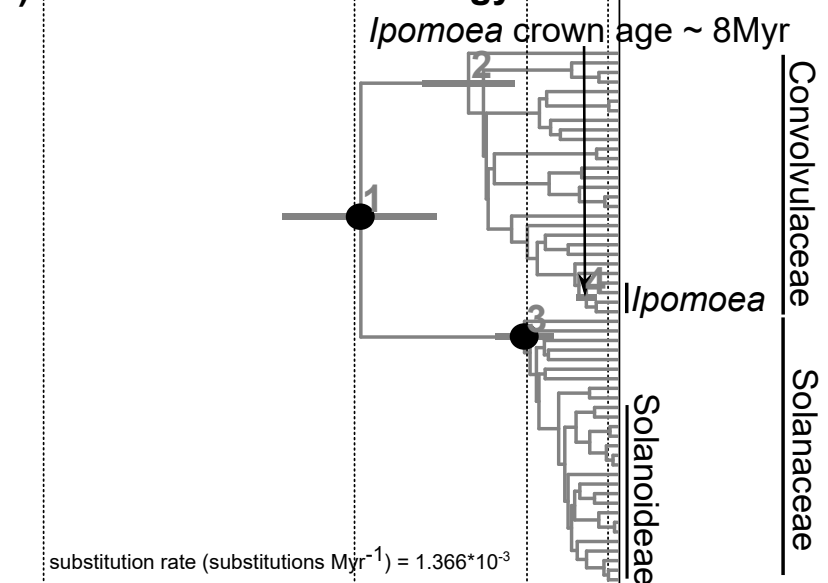

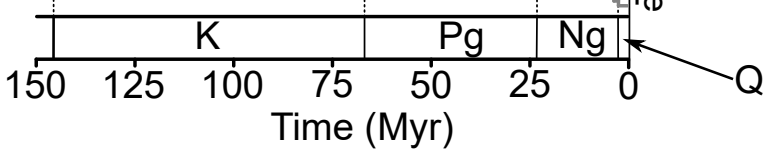




\section{Assumptions about $t$}

\section{Fossil calibrations}

- Used different calibration strategies

- These affect estimates for the age of Ipomoea, but not relative clade ages within Ipomoea, because no fossil calibrations are implemented within Ipomoea

\section{Diversification paramaters}

- Assumptions about diversification paramaters affect expected $t$ for each branch

- Performed analyses in treePL no assumptions about diversification parameters

- Performed analyses in RevBayes with:

1. many taxa - assumption of constant diversification parameters affects expected $t$ for each branch

2. three taxa - assumptions about diversification parameters do not affect expected $t$ for each branch

\section{Divergence time estimation error}

- Erroneous estimates for the age of Ipomoea will affect inferences of diversification parameters throughout Ipomoea

- Took uncertainty in age estimates for the age of Ipomoea into account when discussing inferred diversification parameters

- Error in estimates of relative clade ages within Ipomoea can affect inferences of branch specific diversification parameters

- To account for uncertainty in relative clade ages, diversification parameters inferred from time-calibrated phylogenies inferred with different smoothing values in treePL

\section{Sequence Alignment}
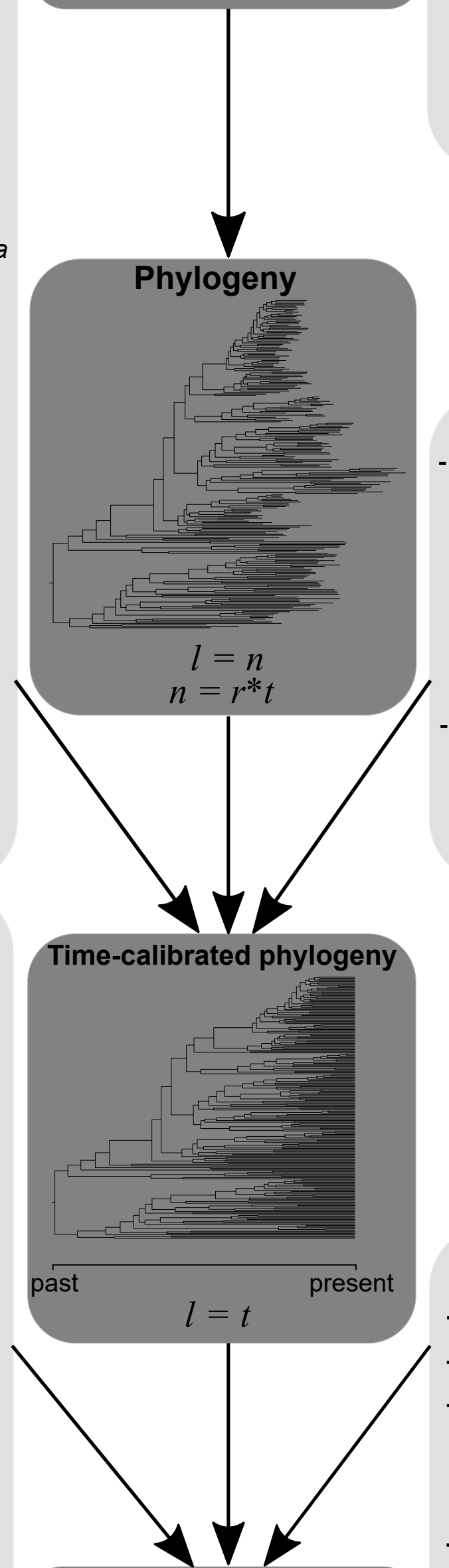

Inferred diversification parameters

\section{Key}

$l=$ branch lengths

$n=$ number of substitutions

$r=$ substitution rate

$t=$ time

\section{Assumptions about $r$}

- Made different assumptions about the magnitude of amongbranch-substitution-rate-variation by using:

1. different smoothing values in treePL

2. either a strict clock or a relaxed clock in RevBayes

- Calculated a value for $r$ to satisfy a timeframe in which $I$. batatas evolved in human times. Could determine whether this value is realistic.

\section{Incomplete sampling}

- Not all extant taxa are sampled

- No extinct taxa are sampled

- Can only partially account for these two forms of incomplete sampling when inferring diversification parameters

- Given incomplete sampling can only partially be accounted for, we discussed its implications in detail in our discussion 

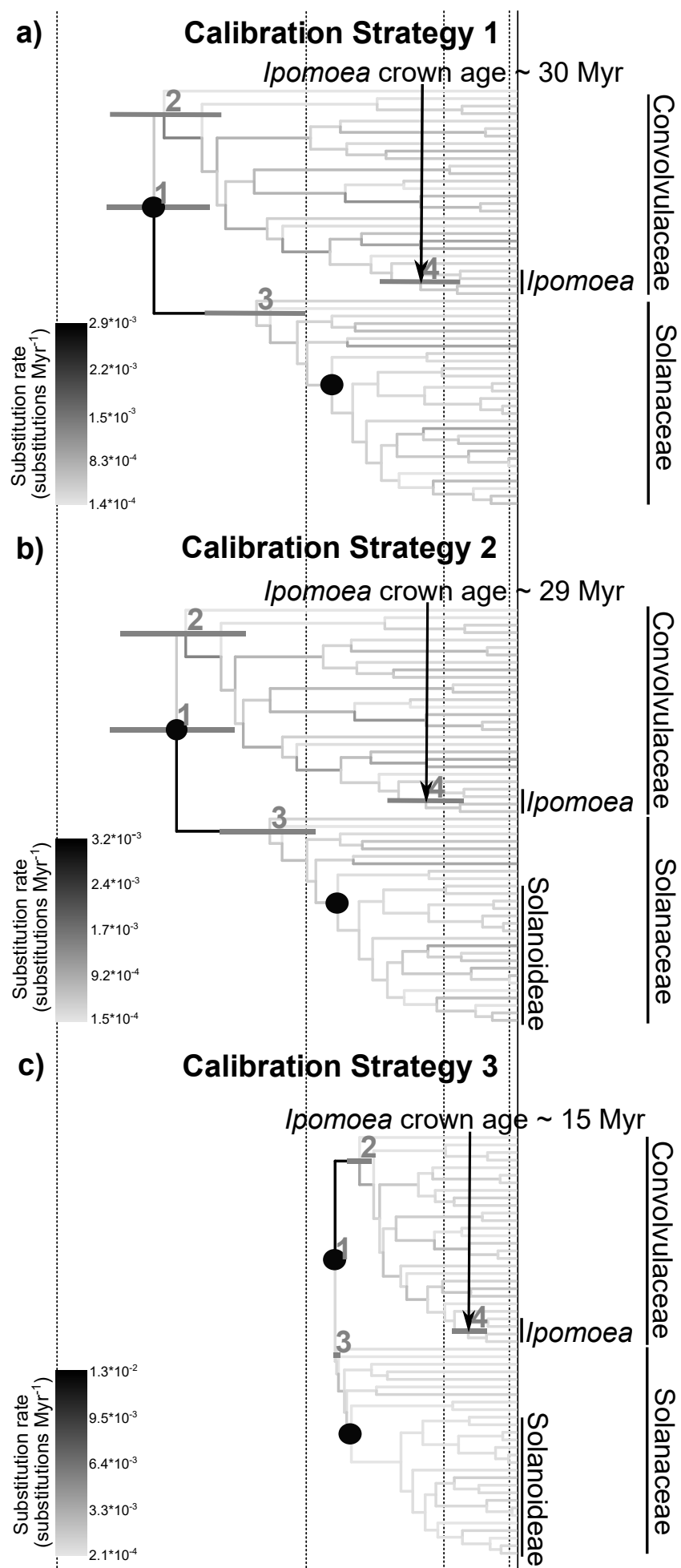

d) Calibration Strategy 4

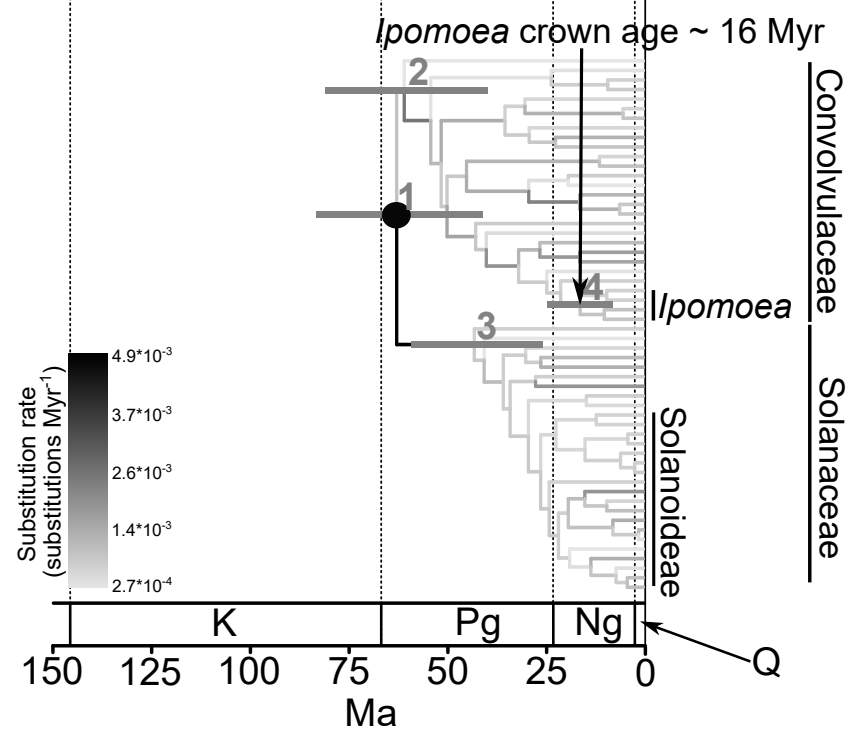



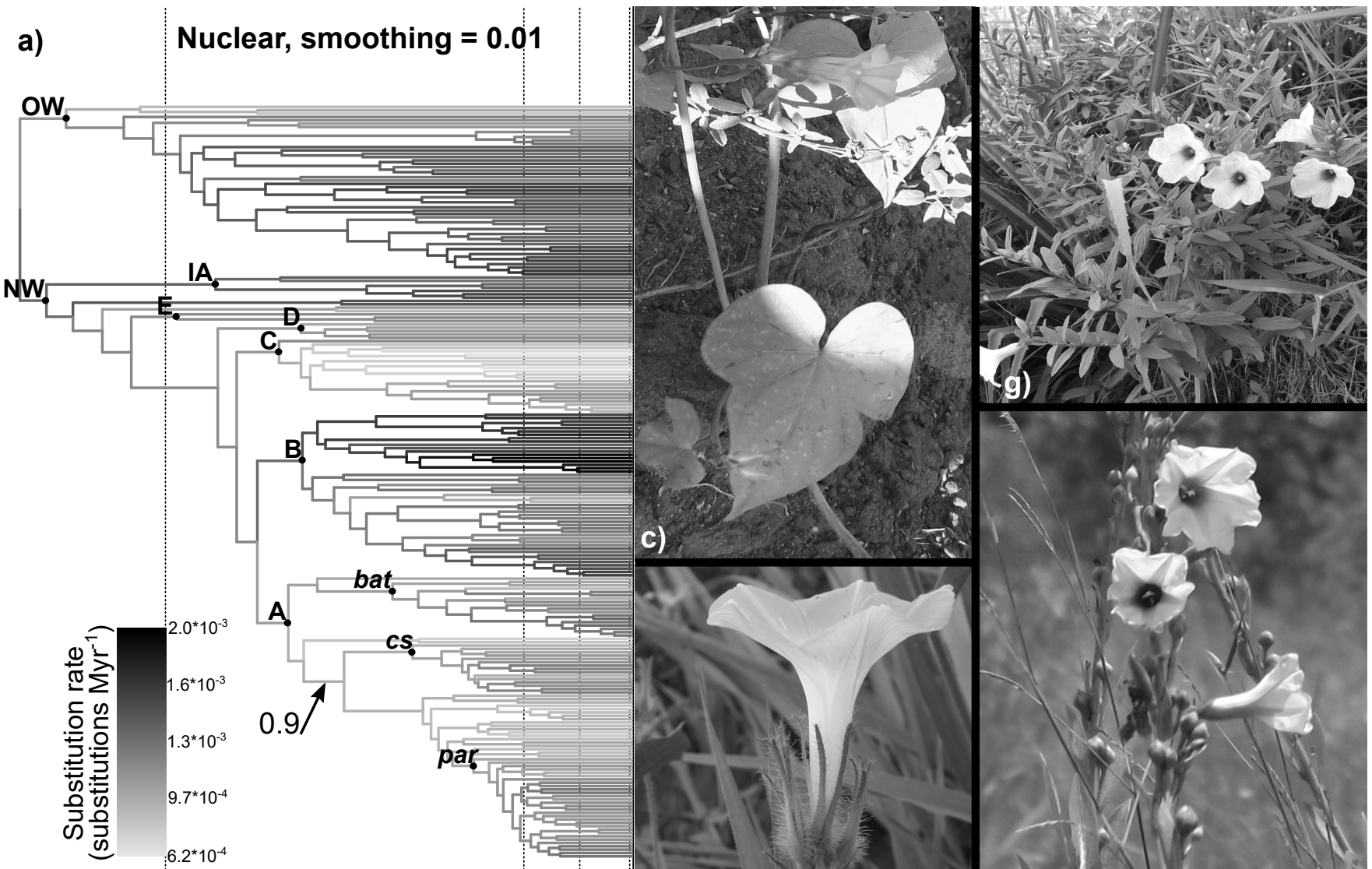

b) Chloroplast, smoothing $=10000$

d)
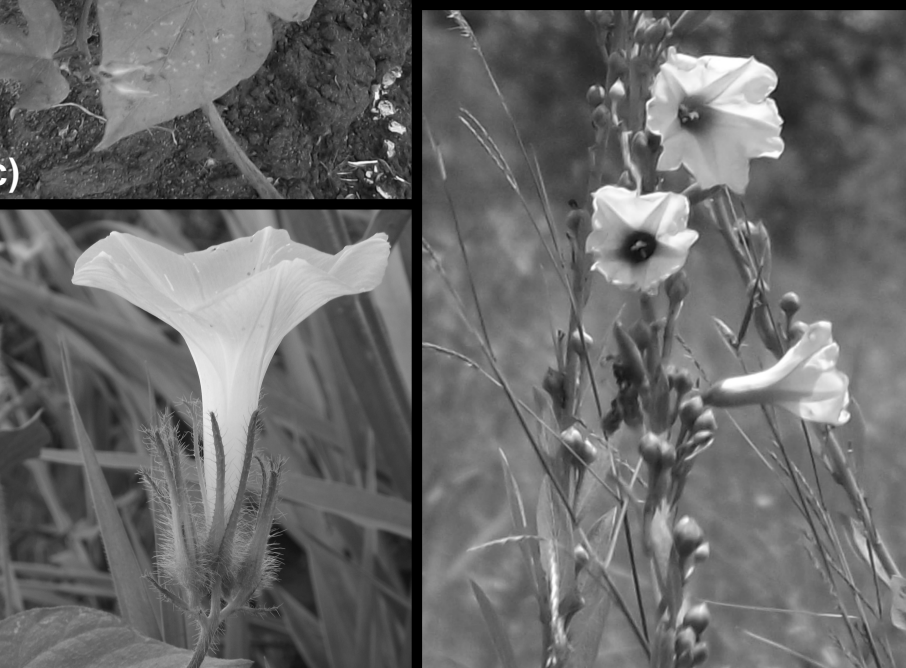

b)
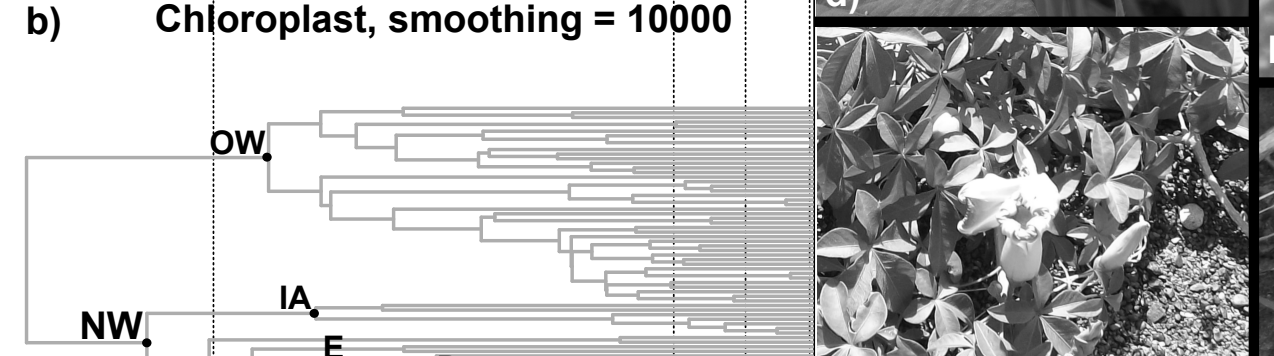

h)
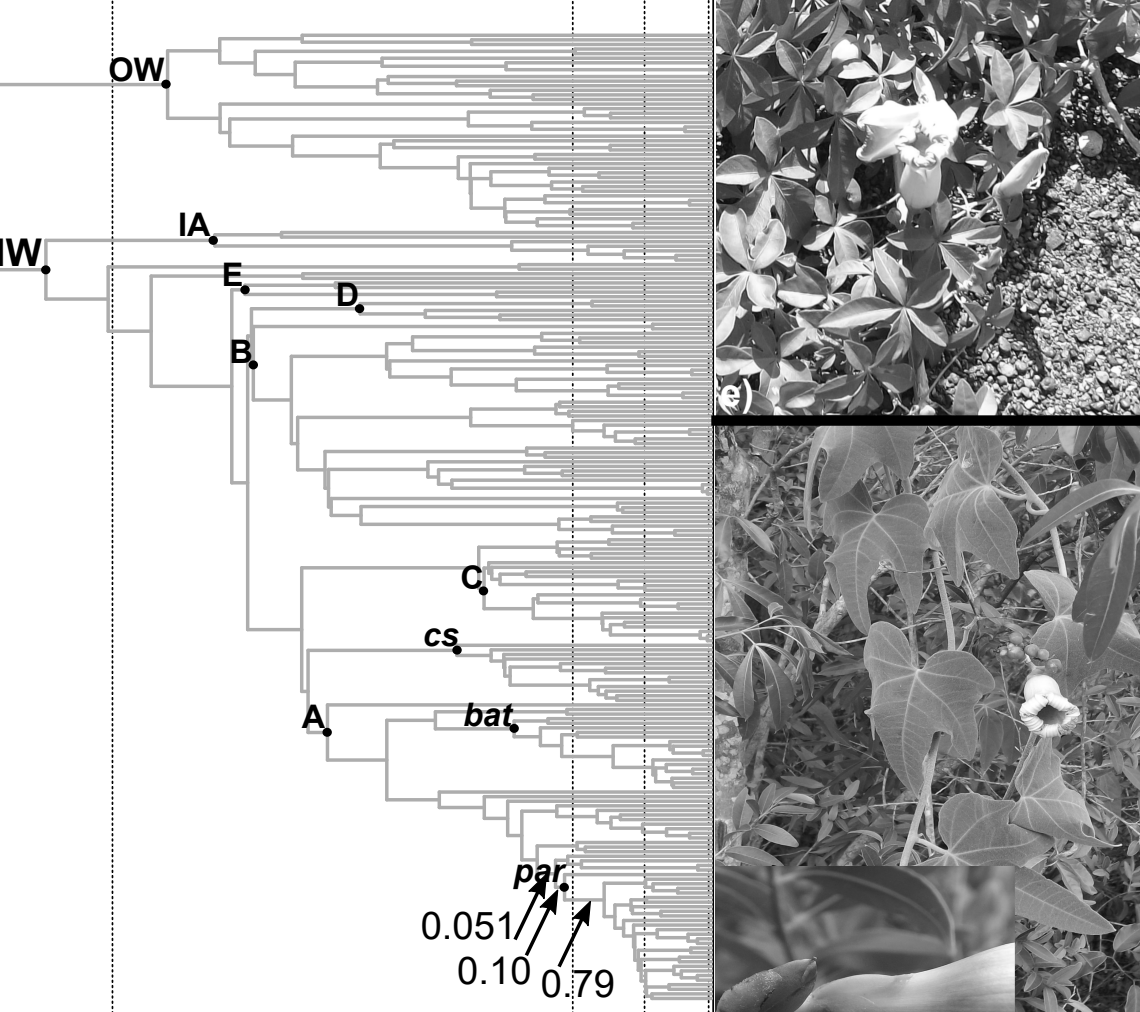

substitution rate (substitutions $\mathrm{Myr}^{-1}$ ) $=1.81 * 10^{-4}$

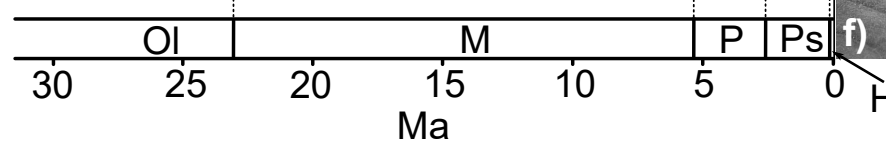


a)

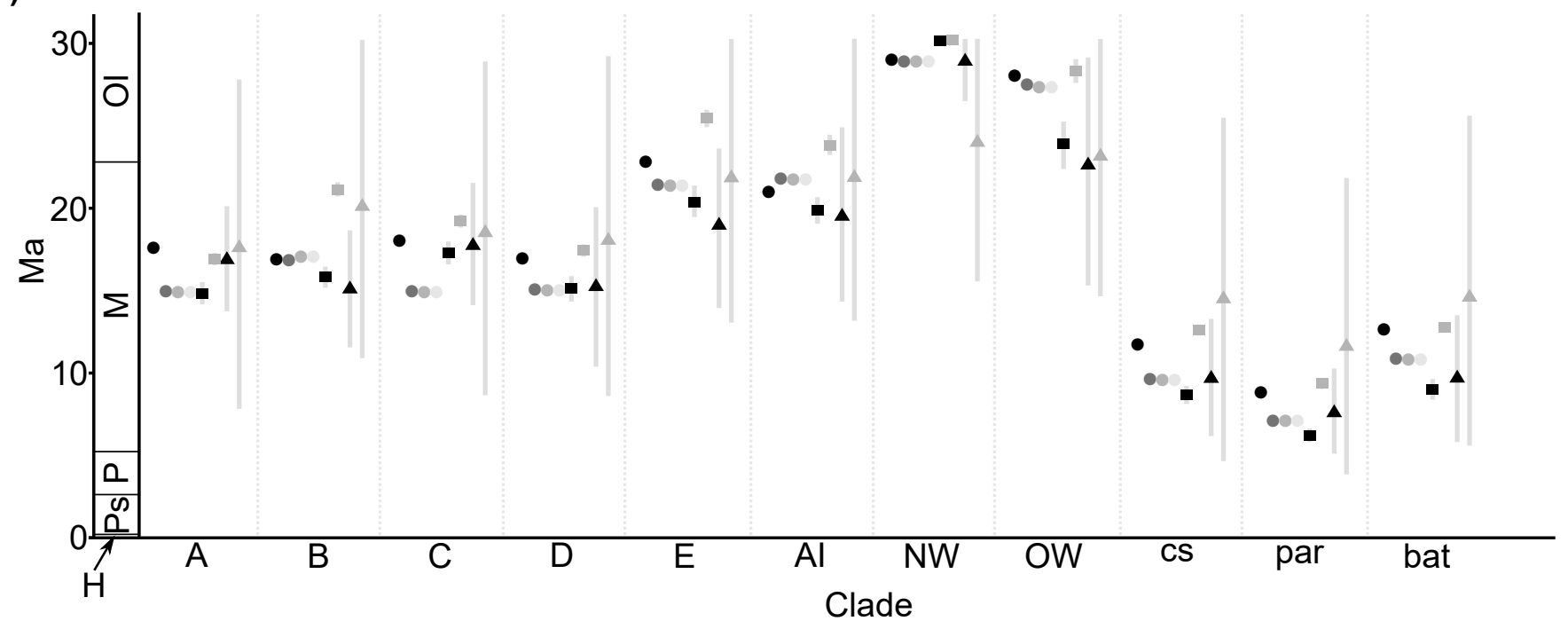

b)

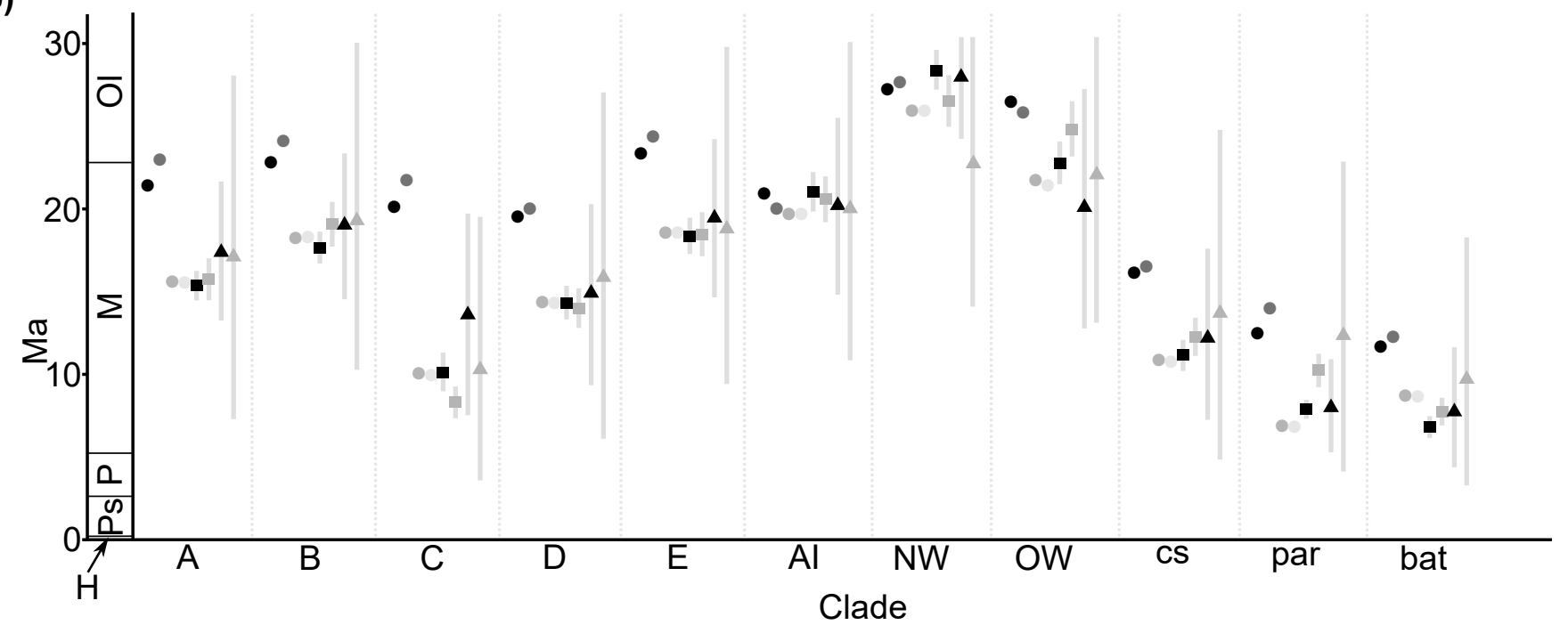

\section{Key}

- treePL, smoothing $=0.01$

- treePL, smoothing $=1$

- treePL, smoothing $=100$ treePL, smoothing $=10000$

- RevBayes, skeletal, strick clock

- RevBayes, three-taxon, strict clock

- RevBayes, skeletal, UCLN relaxed clock

$\triangle$ RevBayes, three-taxon, UCLN relaxed clock 


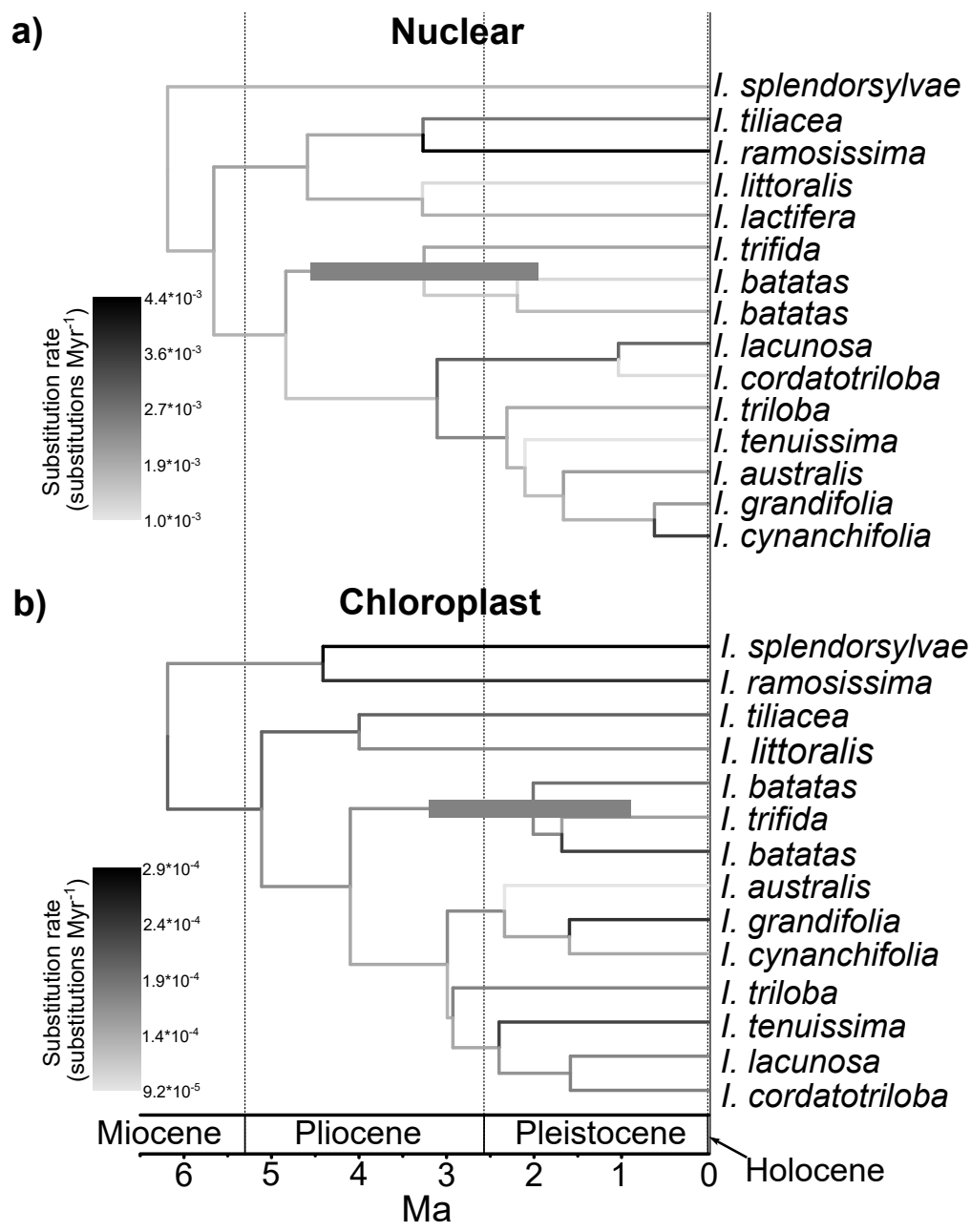


a)

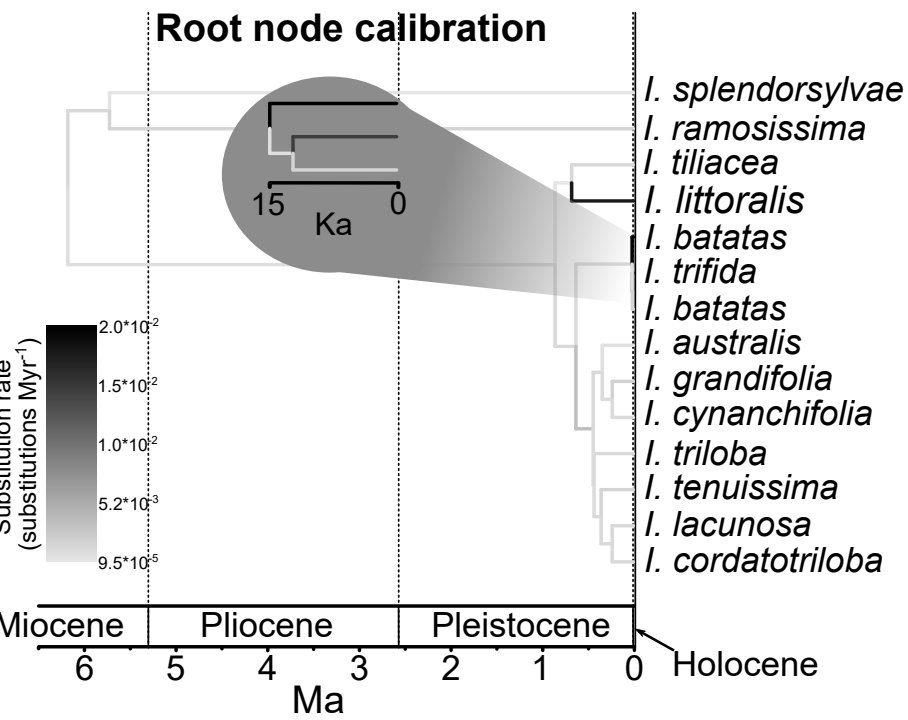

b)

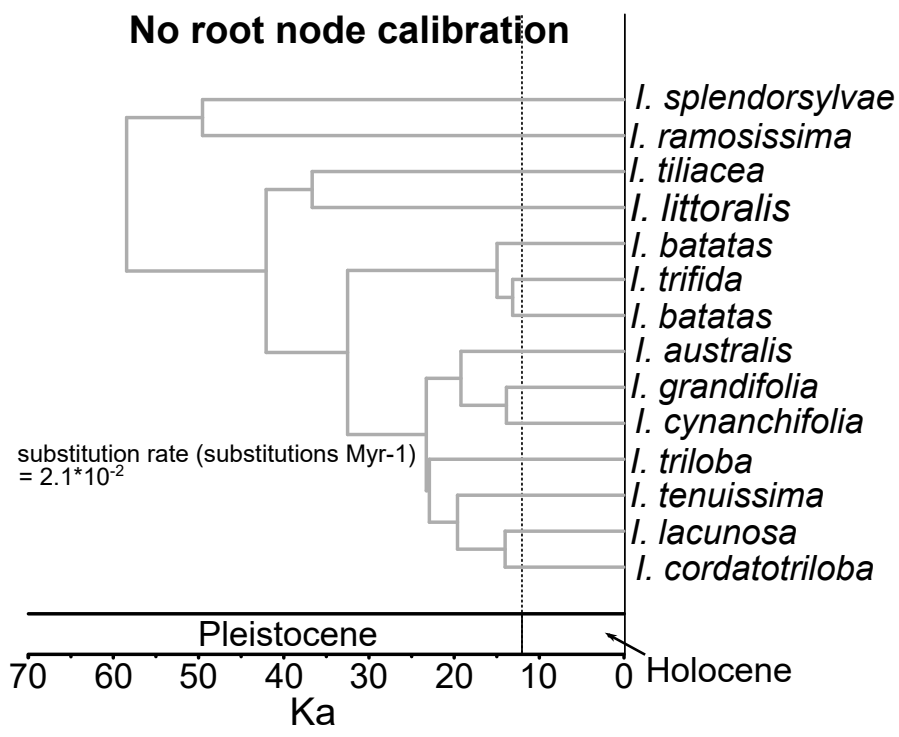




\section{Assumptions about $t$}

\section{Fossil calibrations}

- Used different calibration strategies

- These affect estimates for the age of Ipomoea, but not relative clade ages within Ipomoea, because no fossil calibrations are implemented within Ipomoea

\section{Diversification paramaters}

- Assumptions about diversification paramaters affect expected $t$ for each branch

- Performed analyses in treePL no assumptions about diversification parameters

- Performed analyses in RevBayes with:

1. many taxa - assumption of constant diversification parameters affects expected $t$ for each branch

2. three taxa - assumptions about diversification parameters do not affect expected $t$ for each branch

\section{Divergence time estimation error}

- Erroneous estimates for the age of Ipomoea will affect inferences of diversification parameters throughout Ipomoea

- Took uncertainty in age estimates for the age of Ipomoea into account when discussing inferred diversification parameters

- Error in estimates of relative clade ages within Ipomoea can affect inferences of branch specific diversification parameters

- To account for uncertainty in relative clade ages, diversification parameters inferred from time-calibrated phylogenies inferred with different smoothing values in treePL

\section{Sequence Alignment}
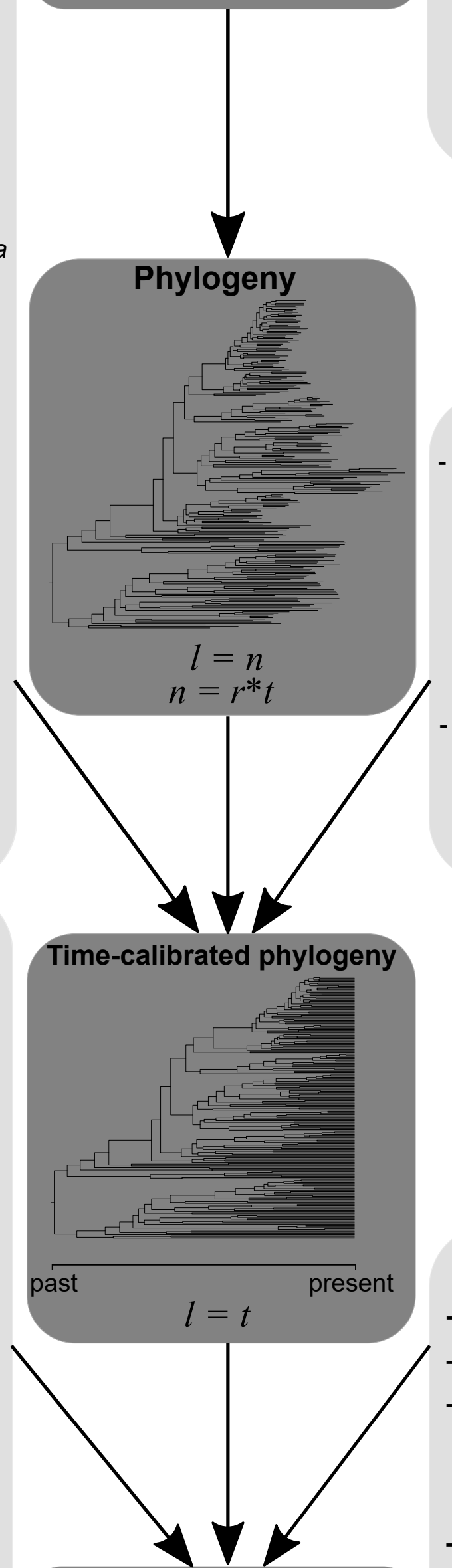

Inferred diversification parameters

\section{Key}

$l=$ branch lengths

$n=$ number of substitutions

$r=$ substitution rate

$t=$ time

\section{Assumptions about $r$}

- Made different assumptions about the magnitude of amongbranch-substitution-rate-variation by using:

1. different smoothing values in treePL

2. either a strict clock or a relaxed clock in RevBayes

- Calculated a value for $r$ to satisfy a timeframe in which $I$. batatas evolved in human times. Could determine whether this value is realistic.

\section{Incomplete sampling}

- Not all extant taxa are sampled

- No extinct taxa are sampled

- Can only partially account for these two forms of incomplete sampling when inferring diversification parameters

- Given incomplete sampling can only partially be accounted for, we discussed its implications in detail in our discussion 
Figure 2
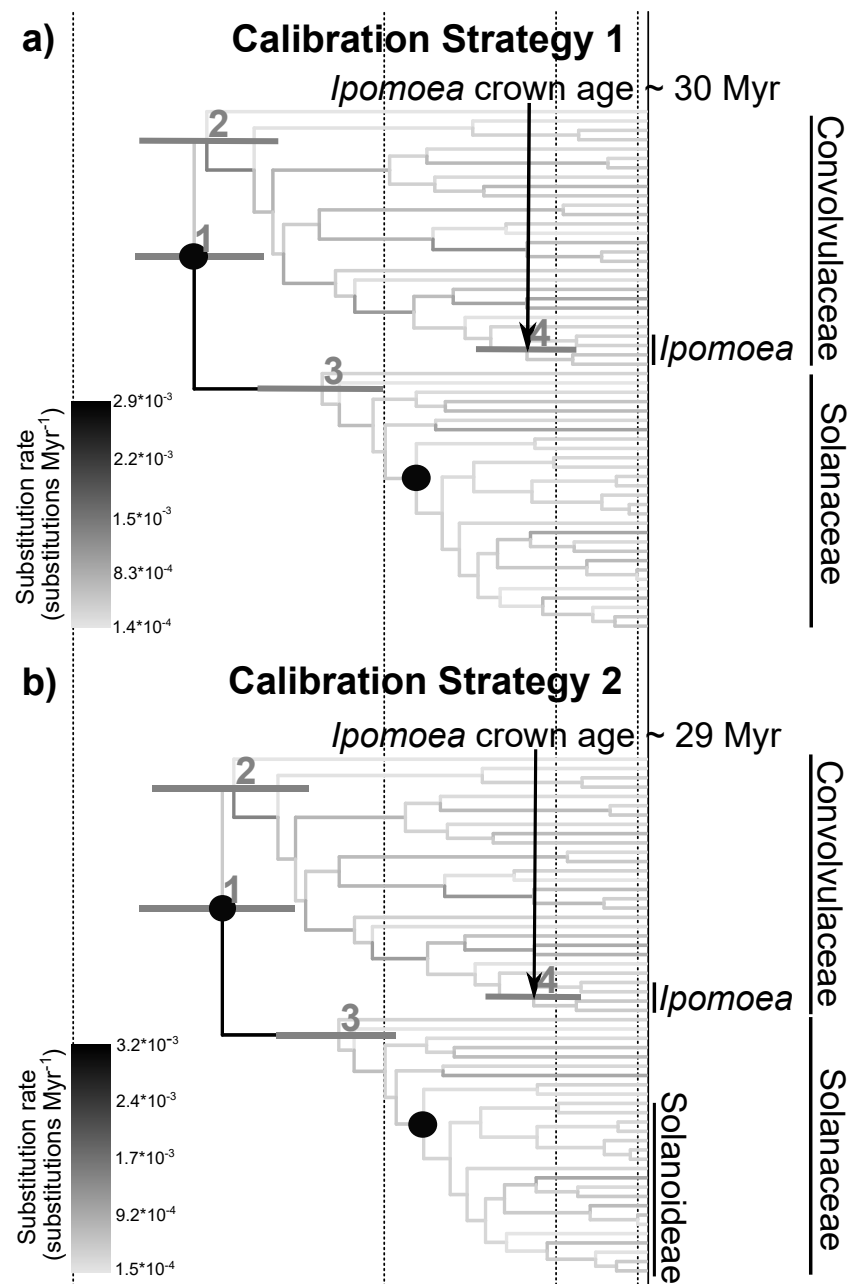

C) Calibration Strategy 3 Ipomoea crown age $\sim 15 \mathrm{Myr}$

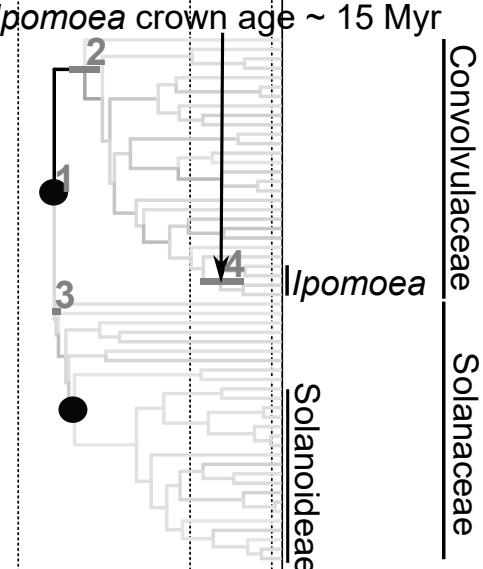

d) Calibration Strategy 4

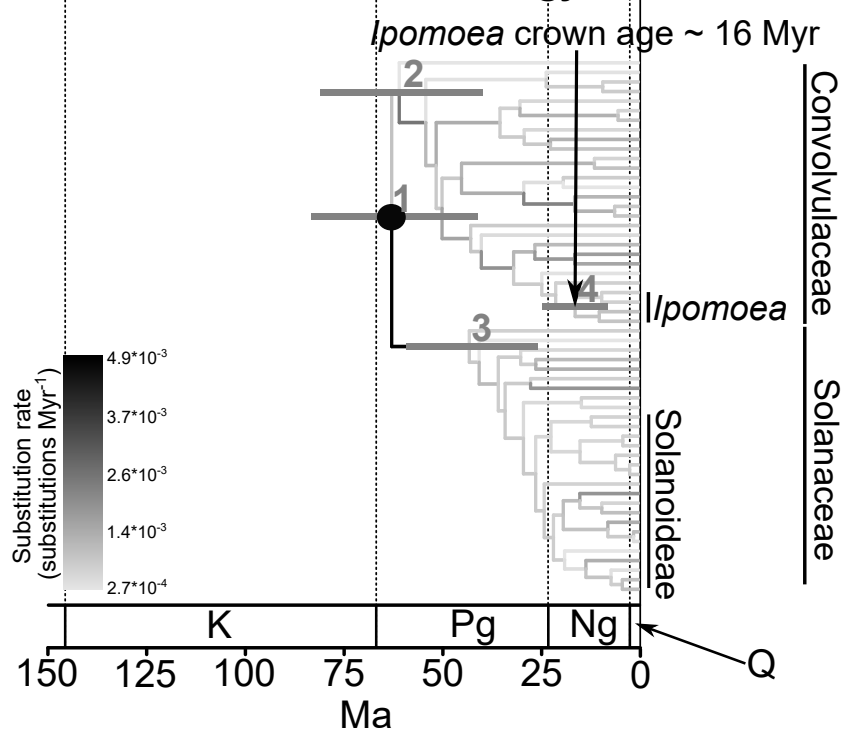



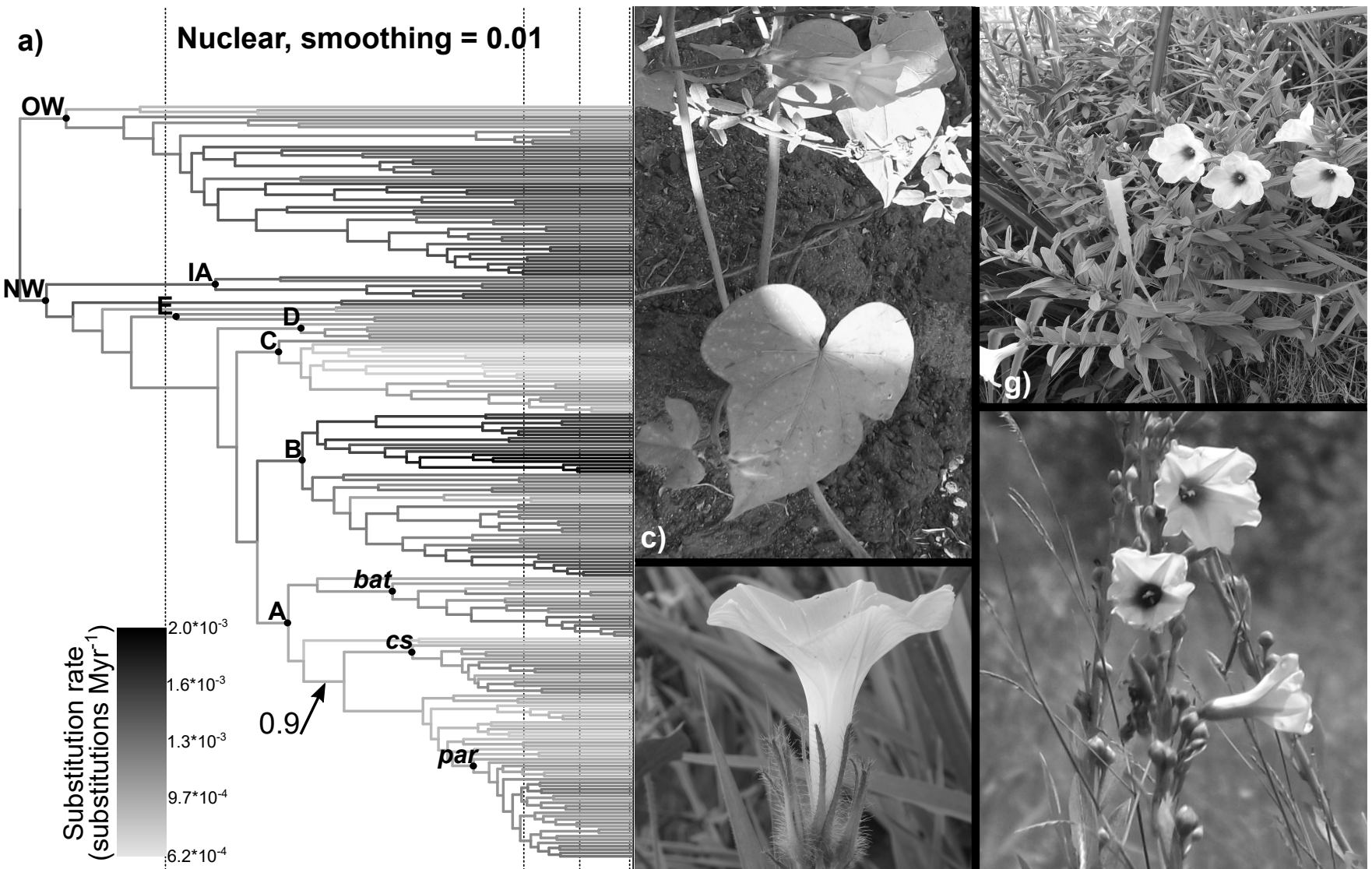

b) Chloroplast, smoothing $=10000$

d)
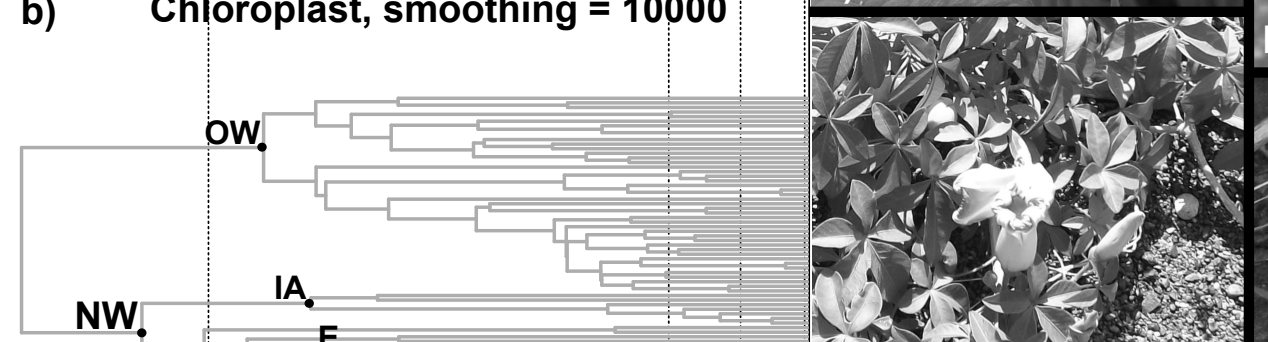

h) 6
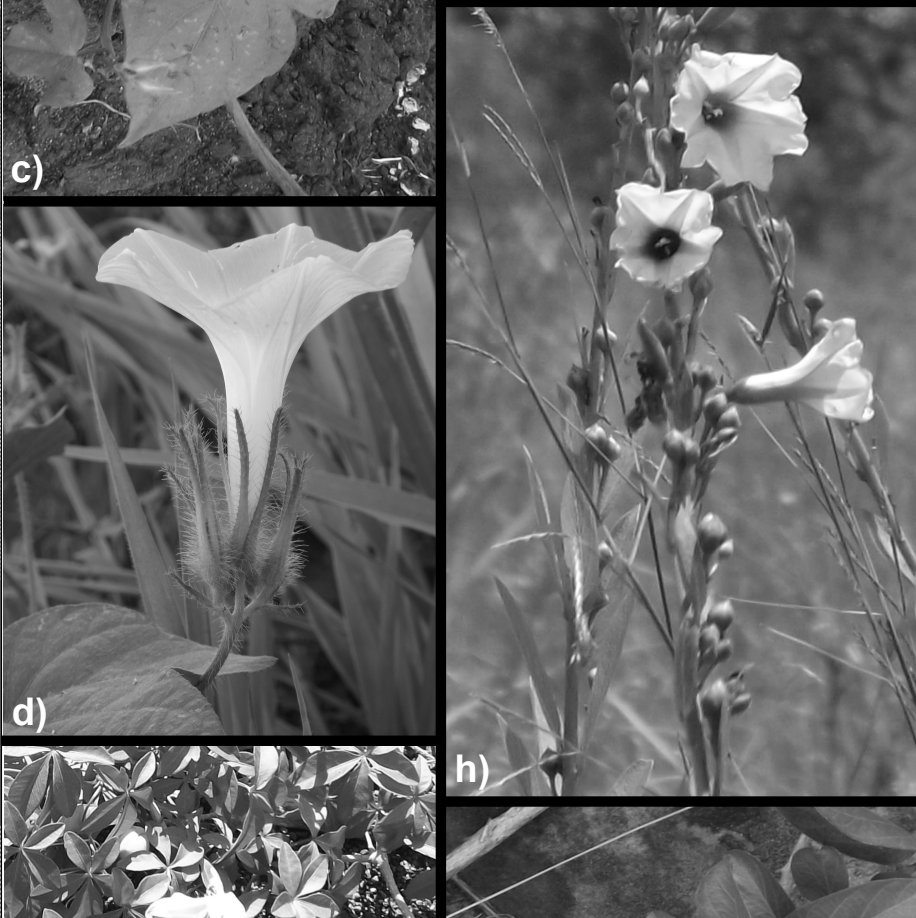

NW
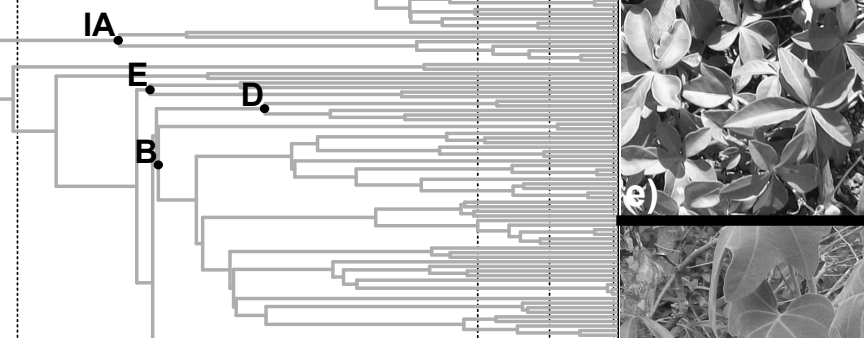
a)
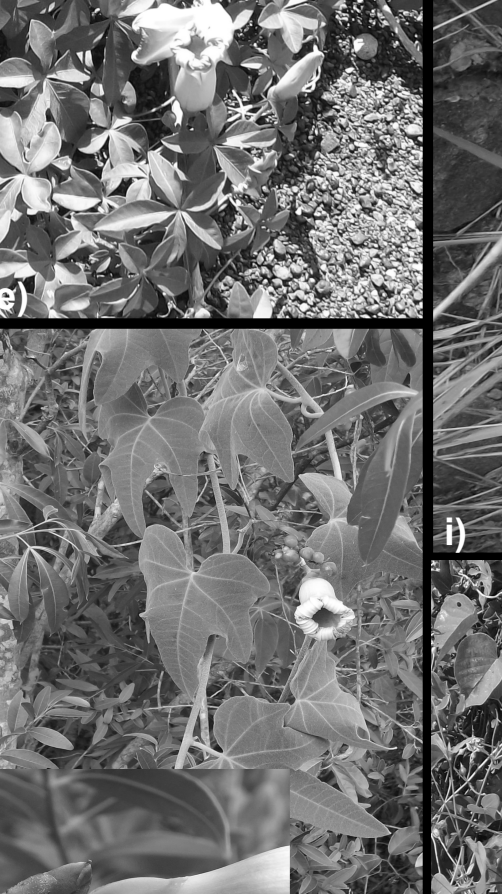

substitution rate (substitutions $\left.\mathrm{Myr}^{-1}\right)=1.81 * 10^{-4}$

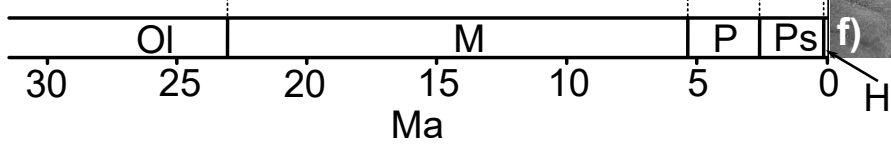


Figuffe 4

a)

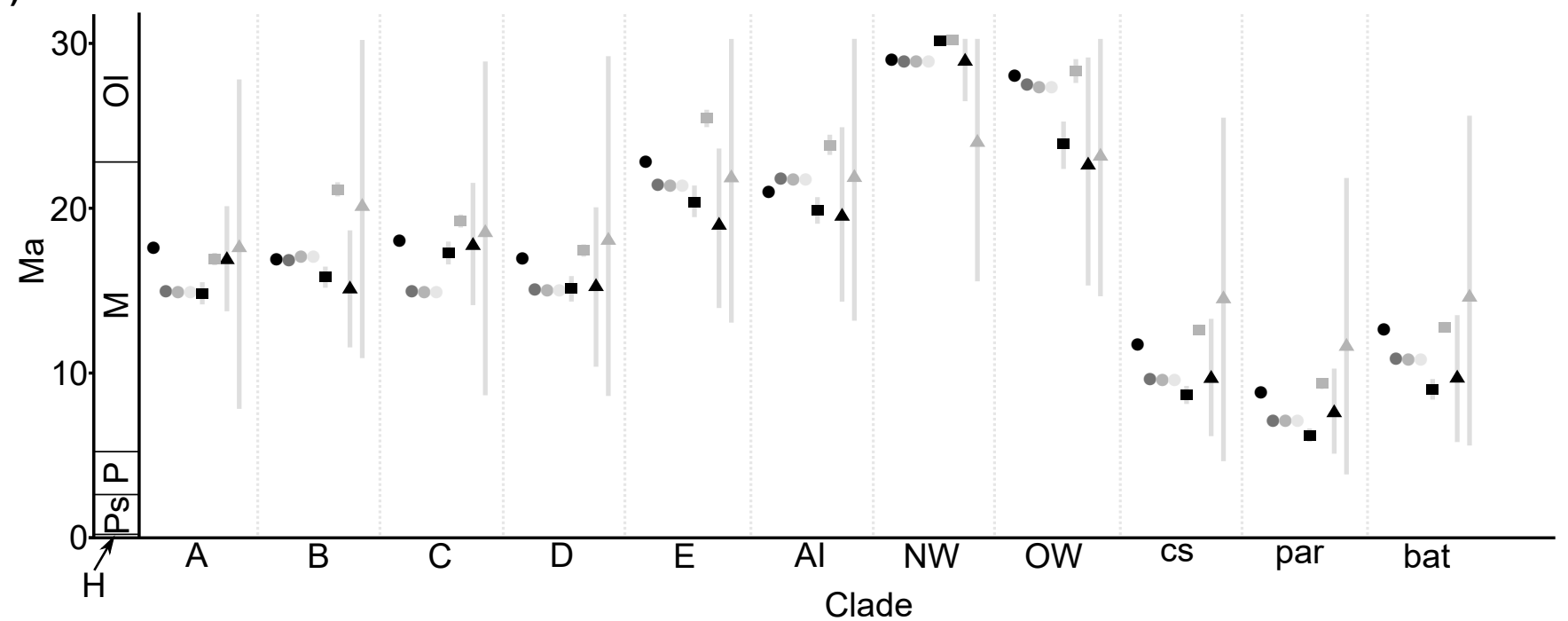

b)

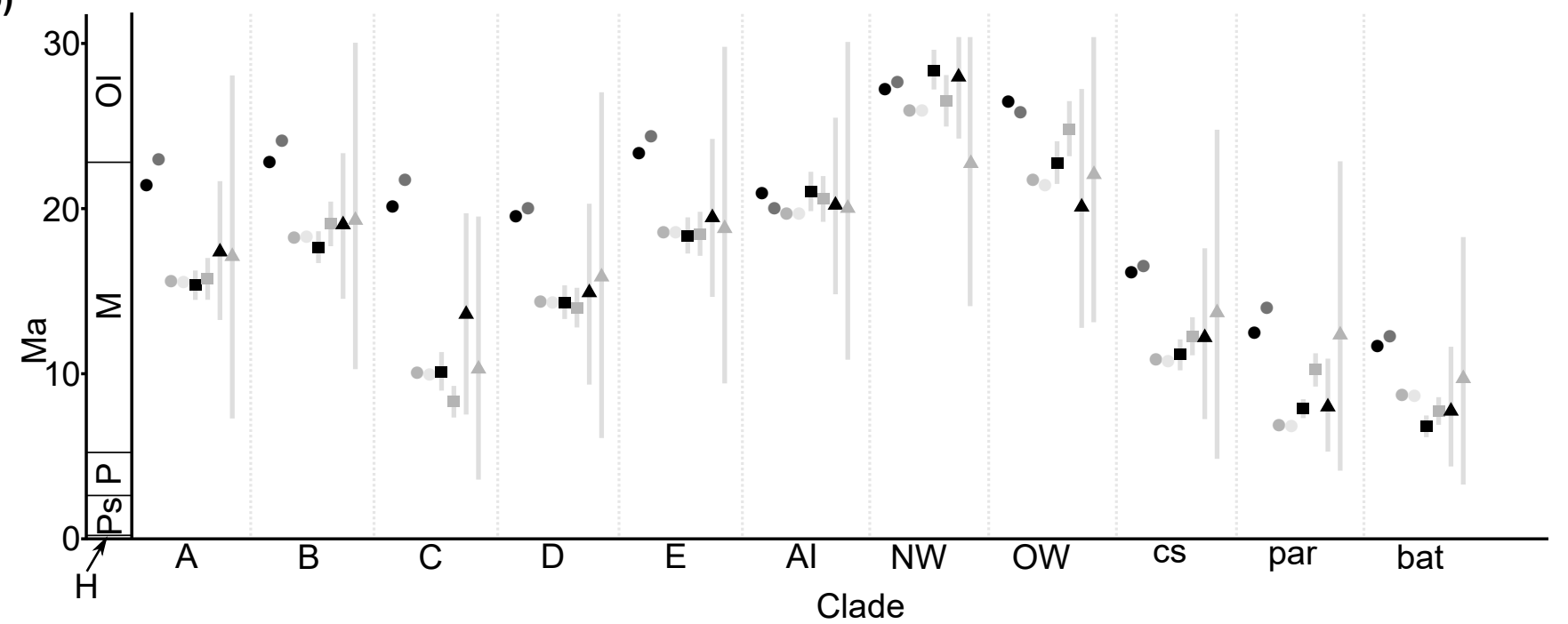

\section{Key}

- treePL, smoothing $=0.01$

- treePL, smoothing $=1$

- treePL, smoothing $=100$ treePL, smoothing $=10000$

- RevBayes, skeletal, strick clock

- RevBayes, three-taxon, strict clock

- RevBayes, skeletal, UCLN relaxed clock

$\triangle$ RevBayes, three-taxon, UCLN relaxed clock 


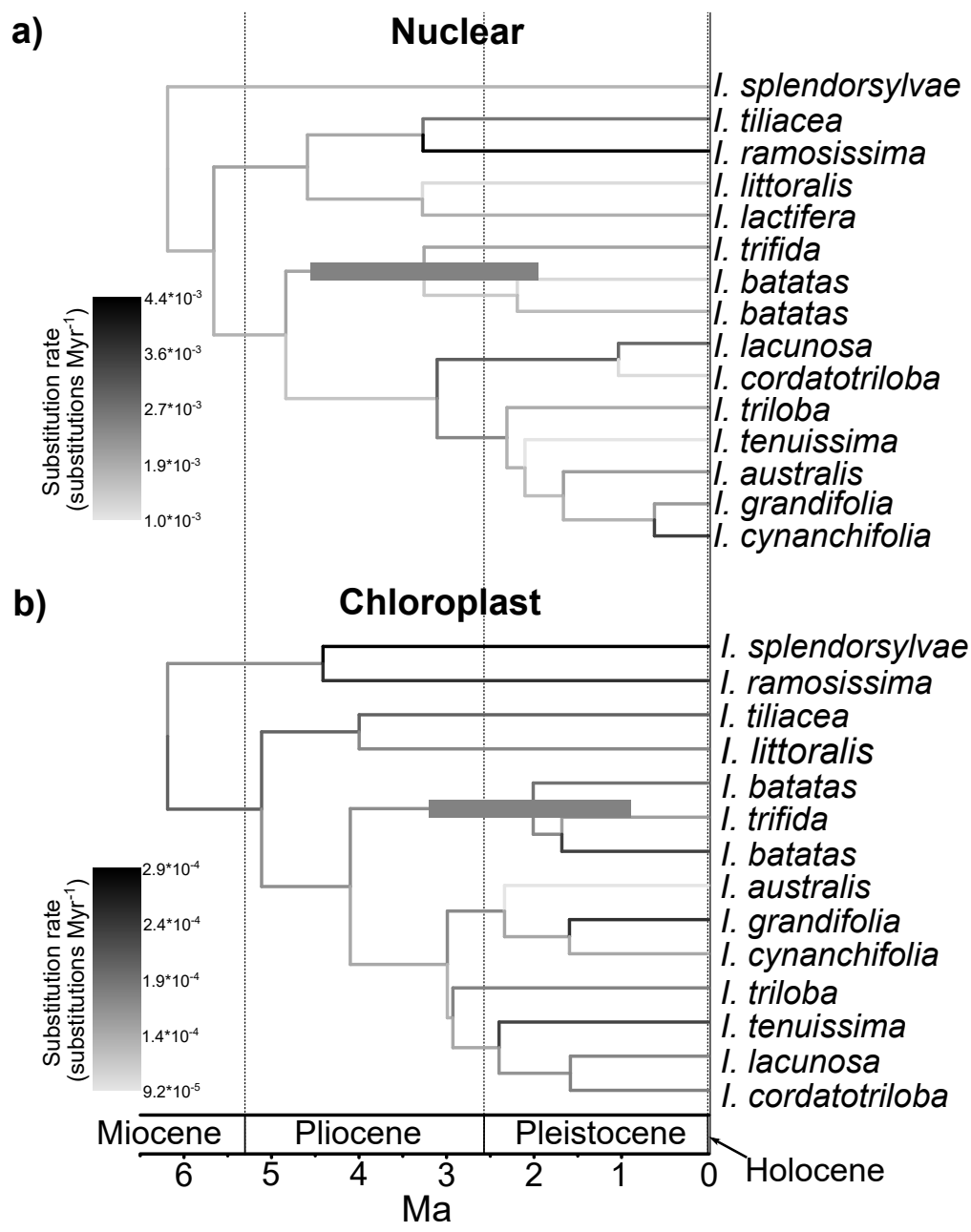


a)

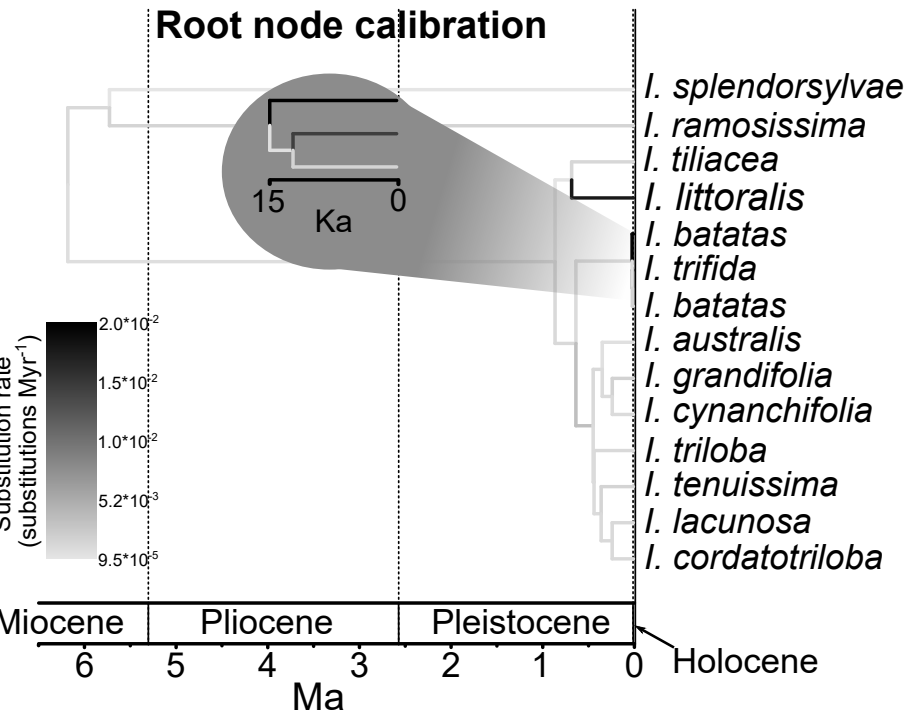

b)

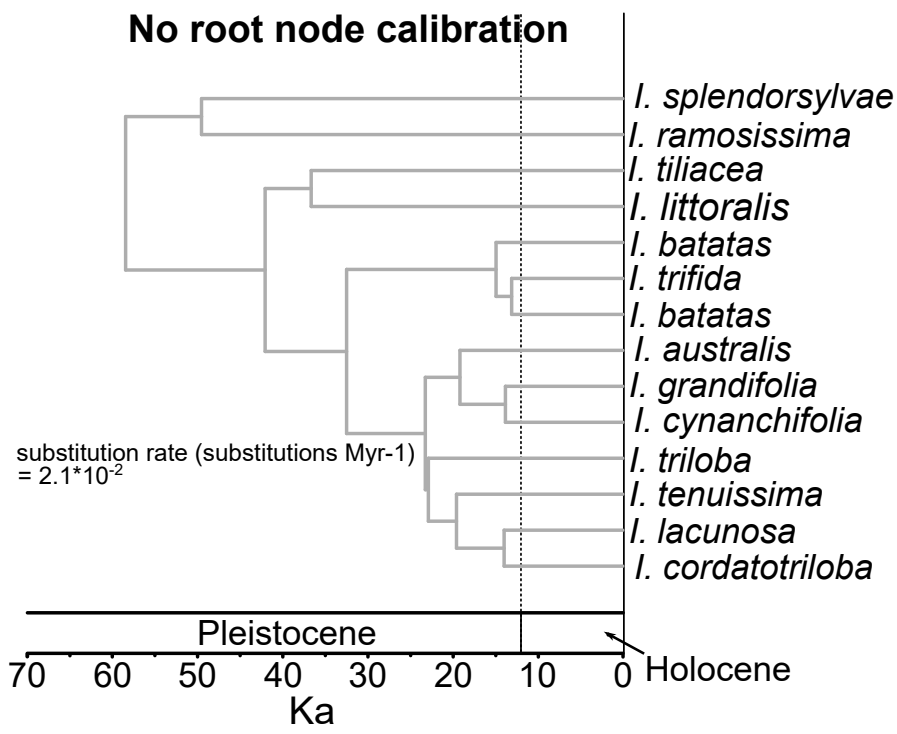


a)

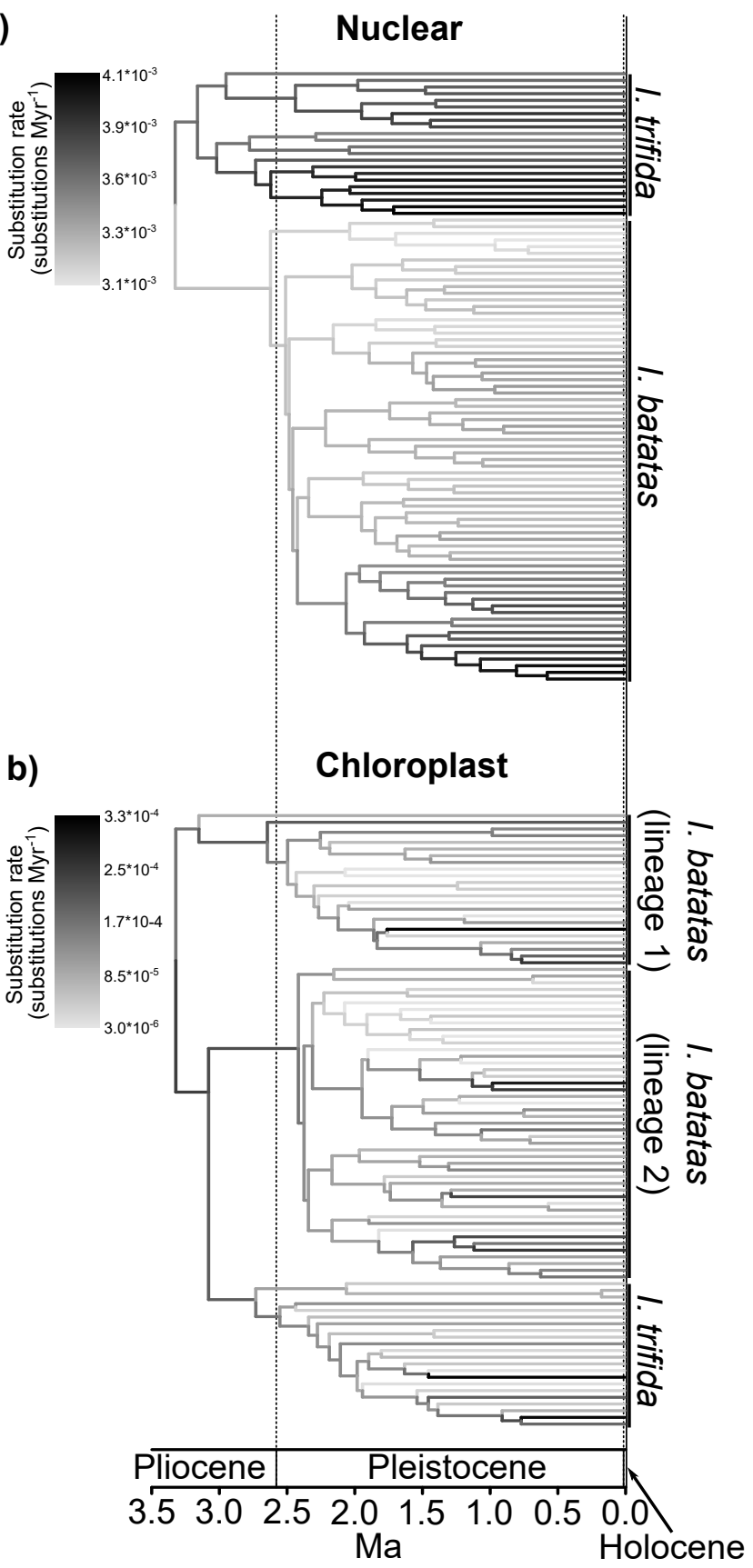


Click here to access/download RDM Data Profile XML DataProfile_4325731.xml 
The manuscript was written by Tom Carruthers and commented on by PMR and RWS. Tom Carruthers did the analyses and all authors contributed to the intellectual framework developed for the study of Ipomoea from which this work emerged. 